\title{
QUASI-POISSON STRUCTURES ON REPRESENTATION SPACES OF SURFACES
}

\author{
GWÉNAËL MASSUYEAU AND VLADIMIR TURAEV
}

\begin{abstract}
Given an oriented surface $\Sigma$ with base point $* \in \partial \Sigma$, we introduce for all $N \geq 1$, a canonical quasi-Poisson bracket on the space of $N$-dimensional linear representations of $\pi_{1}(\Sigma, *)$. Our bracket extends the well-known Poisson bracket on $\mathrm{GL}_{N}$-invariant functions on this space. Our main tool is a natural structure of a quasi-Poisson double algebra (in the sense of M. Van den Bergh) on the group algebra of $\pi_{1}(\Sigma, *)$.
\end{abstract}

\section{INTRODUCTION}

The representation space $\mathcal{H}=\operatorname{Hom}(\pi, G)$ consisting of all homomorphisms from the fundamental group $\pi$ of a compact oriented surface to a Lie group $G$ is a rich source of geometry. The group $G$ acts on $\mathcal{H}$ by conjugations and the quotient $\mathcal{H} / G$ can be identified with a moduli space of flat connections and with a moduli space of holomorphic vector bundles (for appropriate $G$ ). For closed surfaces, the space $\mathcal{H} / G$ carries symplectic geometry. The classical instances are the Weil-Petersson symplectic structure on the Teichmüller space (for $G=\operatorname{PSL}(2, \mathbb{R})$ ) and the Atiyah-Bott symplectic structure for compact $G$ endowed with a nondegenerate $\operatorname{Ad}(G)$-invariant symmetric bilinear form on the corresponding Lie algebra. A systematic approach to the symplectic structure on $\mathcal{H} / G$ was introduced by W. Goldman Go1, Go2 in extension of the work of S. Wolpert Wo. Goldman defined a Lie bracket in the free abelian group generated by the set of conjugacy classes of elements of $\pi$ and used it to compute the Poisson structure on $\mathcal{H} / G$ induced by the symplectic structure. Surfaces with boundary have a canonical Poisson structure on the quotient $\mathcal{H} / G$. It was described in [FR in terms of ciliated fat graphs and in GHJW] in terms of group systems, see also $\mathrm{AMM}$, [La and the surveys $\mathrm{Au}$, Go3, $\mathrm{Hu}$.

In this paper we show that for surfaces with boundary and $G=\mathrm{GL}_{N}(\mathbb{R})$ with $N \geq 1$, there is a canonical Poisson-type structure on $\mathcal{H}$. More precisely, consider a compact oriented surface $\Sigma$ with non-void boundary. Set $\pi=\pi_{1}(\Sigma, *)$ with $* \in \partial \Sigma$ and $\mathcal{H}=\operatorname{Hom}(\pi, G)$. Since $\pi$ is a free group of a finite rank, $n$, a choice of a basis of $\pi$ yields a bijection $\mathcal{H} \cong G^{n}$. This induces a structure of a smooth manifold on $\mathcal{H}$ independent of the choice of the basis. Moreover, the action of $G$ on $\mathcal{H}$ by conjugations is smooth. A. Alekseev, Y. Kosmann-Schwarzbach, and E. Meinrenken [AKsM] introduced a notion of a quasi-Poisson structure on a smooth manifold endowed with a smooth action of a Lie group. We construct here a canonical quasi-Poisson structure on $\mathcal{H}$. One may think of this structure as of a skew-symmetric bracket $\{-,-\}$ in the algebra $C^{\infty}(\mathcal{H})$ of smooth $\mathbb{R}$-valued functions on $\mathcal{H}$ satisfying the Leibniz identity and the modified Jacobi identity

$$
\{f,\{g, h\}\}+\{g,\{h, f\}\}+\{h,\{f, g\}\}=\phi(f, g, h)
$$


for any $f, g, h \in C^{\infty}(\mathcal{H})$. Here $\phi \in \Lambda^{3} \mathfrak{g l}_{N}(\mathbb{R})$ is the Cartan trivector and the action of the Lie algebra $\mathfrak{g l}_{N}(\mathbb{R})$ of $G=\mathrm{GL}_{N}(\mathbb{R})$ on $C^{\infty}(\mathcal{H})$ is induced by the action of $G$ on $\mathcal{H}$ by conjugations. Both $G$ and the group $\operatorname{Homeo}(\Sigma, *)$ of isotopy classes of orientation-preserving self-homeomorphisms of the pair $(\Sigma, *)$ act on $\mathcal{H}$ by bracketpreserving diffeomorphisms. In particular, the bracket is preserved under the Dehn twists about simple closed curves in $\Sigma$. More generally, an orientation-preserving basepoint-preserving homeomorphism of surfaces induces a diffeomorphism of the corresponding representation spaces commuting with the action of $G$ and preserving the quasi-Poisson bracket.

The usual Poisson structure on $\mathcal{H} / G$ is determined by our quasi-Poisson structure on $\mathcal{H}$ as follows. By a smooth function on $\mathcal{H} / G$ we mean a $G$-invariant smooth function on $\mathcal{H}$. The subalgebra $C^{\infty}(\mathcal{H})^{G} \subset C^{\infty}(\mathcal{H})$ of smooth functions on $\mathcal{H} / G$ is closed under our quasi-Poisson bracket $\{-,-\}$ and the restriction of $\{-,-\}$ to $C^{\infty}(\mathcal{H})^{G}$ is a Poisson bracket. The latter bracket divided by two is the usual Poisson bracket in the algebra of smooth functions on $\mathcal{H} / G$.

Quasi-Poisson structures on $\mathcal{H} \cong G^{n}$ were first constructed in $\mathrm{AKsM}$. The approach of [AKsM] consists in producing explicit quasi-Poisson structures on $G$ and on $G \times G$, then combining copies of these structures into a quasi-Poisson structure on $G^{n}$ by a process called "fusion", and finally identifying $\mathcal{H}$ with $G^{n}$ via a choice of a basis of $\pi$. This construction produces a family of quasi-Poisson structures on $\mathcal{H}$ numerated by certain bases of $\pi$. These structures a priori are not invariant under the action of $\operatorname{Homeo}(\Sigma, *)$. Nevertheless, we show that our quasi-Poisson structure on $\mathcal{H}$ coincides with that of $[\mathrm{AKsM}$ ] for a specific choice of a basis of $\pi$.

Our construction of the quasi-Poisson structure on $\mathcal{H}$ proceeds in two steps. First, we introduce an abstract notion of a quasi-Poisson algebra and show how to turn the coordinate algebra of $\mathcal{H}$ (in the sense of algebraic geometry) into a quasi-Poisson algebra. Then we extend the quasi-Poisson bracket in the coordinate algebra of $\mathcal{H}$ to all smooth functions on $\mathcal{H}$.

The definitions and results introduced at the first step apply to both compact and non-compact surfaces and hold over any commutative ring $\mathbb{K}$ rather than $\mathbb{R}$. To work in this generality, we replace the coordinate algebras as above with more general algebras $A_{N}$ derived from the group ring $A=\mathbb{K} \pi$ of $\pi$. The key new point is a relationship between Fox pairings in $A$ and the theory of quasi-Poisson double brackets due to M. Van den Bergh $\mathrm{VdB}$. Namely, we show that the Fox pairing in $A$ defined in $[\mathrm{Tu}$ induces a quasi-Poisson double bracket in $A$. The Van den Bergh theory, which provides a version of Poisson geometry for non-commutative algebras, produces then a quasi-Poisson structure on $A_{N}$.

The present work opens a number of further directions of study: compute our quasi-Poisson bracket via local geometry of representation spaces; compute the bracket in terms of fat graphs and compare it to the construction of Fock and Rosly [FR]; extend our results to other Lie groups or algebraic groups; etc. In a sequel to the paper the authors will discuss a high-dimensional generalization of the quasi-Poisson bracket in the context of string topology, see Remark [7.4.4.

Our exposition is essentially self-contained and proceeds as follows. We define quasi-Poisson algebras in Section 2. In Section 3 we discuss the algebras $A_{N}$ and formulate our main theorem. The proof of this theorem occupies Sections $47 \mathrm{We}$ recall Van den Bergh's theory of double brackets (Section 4), discuss Fox pairings in Hopf algebras (Section 5), and show how to derive double brackets from Fox 
pairings (Section 6). Then we recall the homotopy intersection pairing of [ $\mathrm{Tu}$ ] and prove the main theorem (Section 7). For compact surfaces, we derive from this theorem a natural quasi-Poisson structure on the representation manifold $\mathcal{H}$ and explicitly compute this structure in certain coordinates on $\mathcal{H}$ (Section 8). In Section 9 we consider moment maps and discuss surfaces without boundary. In Section 10 we extend a part of our constructions to Fuchsian groups. We conclude with two appendices. In Appendix A we discuss in more detail certain group actions and Lie algebra actions on $A_{N}$ introduced in Section 3 . In Appendix B we compare the quasi-Poisson structures on $\mathcal{H}$ introduced in this paper and in AKsM].

Throughout the paper we fix a commutative ring $\mathbb{K}$. Unless stated otherwise, by a module we shall mean a $\mathbb{K}$-module, and by a linear map of modules we mean a $\mathbb{K}$-linear map. By an algebra we shall mean an associative unital $\mathbb{K}$-algebra.

Acknowledgements. The work of G. Massuyeau was partially supported by the French ANR research project ANR-08-JCJC-0114-01. G.M. would like to thank his colleagues in Strasbourg for helpful conversations: P. Baumann, C. Gasbarri, C. Kassel and C. Noot-Huyghe. The work of V. Turaev was partially supported by the NSF grant DMS-0904262. V.T. would like to thank A. Ramadoss for useful discussions.

\section{Quasi-Poisson algebras}

Inspired by the theory of quasi-Poisson manifolds [AKsM], we introduce in this section quasi-Poisson algebras generalizing the familiar Poisson algebras.

2.1. Poisson algebras. Recall that a derivation of an algebra $\mathcal{A}$ is a linear map $d: \mathcal{A} \rightarrow \mathcal{A}$ such that $d(a b)=d(a) b+a d(b)$ for all $a, b \in \mathcal{A}$. A Poisson algebra is an algebra $\mathcal{A}$ endowed with a skew-symmetric bilinear form $\{-,-\}: \mathcal{A} \times \mathcal{A} \rightarrow \mathcal{A}$ which is a derivation in each variable and satisfies the Jacobi identity: for all $a, b, c \in \mathcal{A}$,

$$
\{a,\{b, c\}\}+\{b,\{c, a\}\}+\{c,\{a, b\}\}=0 .
$$

The form $\{-,-\}$ satisfying these conditions is called a Poisson bracket. Note for the record that for all $a, b, c \in \mathcal{A}$,

$$
\{b, a\}=-\{a, b\} \quad \text { and } \quad\{a b, c\}=\{a, c\} b+a\{b, c\} .
$$

We emphasize that we do not require the self-annihilating relation $\{a, a\}=0$ for $a \in \mathcal{A}$. Likewise, speaking about Lie brackets we require skew-symmetry and the Jacobi relation but not the self-annihilating relation. The reader uncomfortable with these conventions may assume from now on that $2 \in \mathbb{K}$ is invertible so that the self-annihilating relation follows from the skew-symmetry.

2.2. Quasi-Poisson $\mathfrak{g}$-algebras. The derivations of an algebra $\mathcal{A}$ form a Lie algebra, $\operatorname{Der}(\mathcal{A})$, with Lie bracket $\left[d_{1}, d_{2}\right]=d_{1} d_{2}-d_{2} d_{1}$ for all $d_{1}, d_{2} \in \operatorname{Der}(\mathcal{A})$. A (left) action of a Lie algebra $\mathfrak{g}$ on $\mathcal{A}$ is a Lie algebra homomorphism $\mathfrak{g} \rightarrow \operatorname{Der}(\mathcal{A})$. Given such an action, we say that $\mathcal{A}$ is an algebra over $\mathfrak{g}$ or, shorter, a $\mathfrak{g}$-algebra. An element $a \in \mathcal{A}$ is $\mathfrak{g}$-invariant if $w a=0$ for all $w \in \mathfrak{g}$. The $\mathfrak{g}$-invariant elements of $\mathcal{A}$ form a subalgebra of $\mathcal{A}$ denoted $\mathcal{A}^{\mathfrak{g}}$. 
An example of a $\mathfrak{g}$-algebra is provided by the tensor algebra $\oplus_{n \geq 0} \mathfrak{g}^{\otimes n}$ where each $w \in \mathfrak{g}$ acts by

$$
w\left(w_{1} \otimes \cdots \otimes w_{n}\right)=\sum_{i=1}^{n} w_{1} \otimes \cdots \otimes w_{i-1} \otimes\left[w, w_{i}\right] \otimes w_{i+1} \otimes \cdots \otimes w_{n}
$$

for any $w_{1}, \ldots, w_{n} \in \mathfrak{g}$. A vector $a \in \mathfrak{g}^{\otimes n}$ is skew-symmetric if any transposition of tensor factors carries $a$ into $-a$. The module of $\mathfrak{g}$-invariant skew-symmetric vectors of $\mathfrak{g}^{\otimes n}$ is denoted by $\wedge_{\mathfrak{g}}^{n}$. We shall be specifically interested in this module for $n=3$. In particular, a nonsingular $\mathfrak{g}$-invariant symmetric bilinear form $\cdot: \mathfrak{g} \times \mathfrak{g} \rightarrow \mathbb{K}$ defines a vector $\phi \in \wedge_{\mathfrak{g}}^{3}$ which is the skew-symmetric trilinear form

$$
\mathfrak{g} \otimes \mathfrak{g} \otimes \mathfrak{g} \longrightarrow \mathbb{K},\left(w_{1}, w_{2}, w_{3}\right) \longmapsto w_{1} \cdot\left[w_{2}, w_{3}\right]
$$

viewed as a skew-symmetric element of $\left(\mathfrak{g}^{*}\right)^{\otimes 3} \simeq \mathfrak{g}^{\otimes 3}$ through the isomorphism $\mathfrak{g} \rightarrow \mathfrak{g}^{*}, w \mapsto w \cdot(-)$. We call $\phi$ the Cartan trivector associated to the form $\cdot$

Fix a Lie algebra $\mathfrak{g}$ and a vector $\phi \in \wedge_{\mathfrak{g}}^{3}$. A $\mathfrak{g}$-algebra $\mathcal{A}$ is quasi-Poisson if $\mathcal{A}$ is endowed with a skew-symmetric bilinear form $\{-,-\}: \mathcal{A} \times \mathcal{A} \rightarrow \mathcal{A}$ which is a derivation in each variable and satisfies the following two identities:

$$
w\{a, b\}=\{w a, b\}+\{a, w b\}
$$

for all $w \in \mathfrak{g}, a, b \in \mathcal{A}$ and

$$
\{a,\{b, c\}\}+\{b,\{c, a\}\}+\{c,\{a, b\}\}=\phi(a, b, c)
$$

for all $a, b, c \in \mathcal{A}$. Here $\phi(a, b, c) \in \mathcal{A}$ is the image of $\phi \in \wedge_{\mathfrak{g}}^{3} \subset \mathfrak{g}^{\otimes 3}$ under the linear map $\mathfrak{g}^{\otimes 3} \rightarrow \mathcal{A}$ carrying $w_{1} \otimes w_{2} \otimes w_{3}$ to $\left(w_{1} a\right)\left(w_{2} b\right)\left(w_{3} c\right)$ for all $w_{1}, w_{2}, w_{3} \in \mathfrak{g}$.

We call the form $\{-,-\}$ a quasi-Poisson bracket in $\mathcal{A}$ associated with $\phi$. Clearly, $\left\{\mathcal{A}^{\mathfrak{g}}, \mathcal{A}^{\mathfrak{g}}\right\} \subset \mathcal{A}^{\mathfrak{g}}$ and the restriction of $\{-,-\}$ to $\mathcal{A}^{\mathfrak{g}}$ is a Poisson bracket in $\mathcal{A}^{\mathfrak{g}}$. Thus, a quasi-Poisson structure in $\mathcal{A}$ induces a Poisson structure in $\mathcal{A}^{\mathfrak{g}}$. For $\mathfrak{g}=\{0\}$, a quasi-Poisson structure in $\mathcal{A}$ is just a Poisson structure in $\mathcal{A}$.

2.3. Quasi-Poisson algebras over Lie pairs. The notion of a quasi-Poisson algebra over a Lie algebra may be generalized bringing a group into the picture. To do it, we introduce the following notion: a Lie pair is a pair $(G, \mathfrak{g})$ where $G$ is a group and $\mathfrak{g}$ is a Lie algebra endowed with a (left) action of $G$ on $\mathfrak{g}$ by Lie algebra automorphisms. The action is denoted by $w \mapsto{ }^{g} w$ for $w \in \mathfrak{g}$ and $g \in G$.

For example, a pair consisting of the trivial group $G=\{1\}$ and any Lie algebra is a Lie pair. The pair consisting of any group and the trivial Lie algebra $\mathfrak{g}=\{0\}$ is a Lie pair. For $\mathbb{K}=\mathbb{R}$, examples of Lie pairs are provided by pairs (a Lie group $G$, the Lie algebra $\mathfrak{g}$ of $G$ with the (left) adjoint action of $G$ ). Further examples of Lie pairs are given in Section 2.4.

An algebra over a Lie pair $(G, \mathfrak{g})$ or, shorter, a $(G, \mathfrak{g})$-algebra is a $\mathfrak{g}$-algebra $\mathcal{A}$ endowed with a (left) action of $G$ by algebra automorphisms such that ${ }^{g_{w}} w(a)=$ $g w\left(g^{-1} a\right)$ for all $w \in \mathfrak{g}, g \in G, a \in \mathcal{A}$. An element $a \in \mathcal{A}$ is $G$-invariant if $g a=a$ for all $g \in G$. The $G$-invariant elements of $\mathcal{A}$ form a subalgebra of $\mathcal{A}$ denoted $\mathcal{A}^{G}$. Note that $\mathcal{A}^{\mathfrak{g}}$ is stable under the action of $G$; generally speaking $\mathcal{A}^{\mathfrak{g}} \neq \mathcal{A}^{G}$.

For example, the tensor algebra $\oplus_{n \geq 0} \mathfrak{g}^{\otimes n}$ is a $(G, \mathfrak{g})$-algebra where $\mathfrak{g}$ acts by (2.2.1) and each $g \in G$ acts by

$$
g\left(w_{1} \otimes w_{2} \otimes \cdots \otimes w_{n}\right)={ }^{g} w_{1} \otimes{ }^{g} w_{2} \otimes \cdots \otimes{ }^{g} w_{n}
$$

for any $w_{1}, \ldots, w_{n} \in \mathfrak{g}$. 
An algebra $\mathcal{A}$ over a Lie pair $(G, \mathfrak{g})$ is quasi-Poisson if $\mathcal{A}$, considered as a $\mathfrak{g}$ algebra, has a quasi-Poisson bracket $\{-,-\}$ associated with a $G$-invariant trivector $\phi \in \wedge_{\mathfrak{g}}^{3}$ and for all $g \in G, a, b \in \mathcal{A}$,

$$
g\{a, b\}=\{g a, g b\} .
$$

For $G=\{1\}$, we recover the notion of a quasi-Poisson algebra over $\mathfrak{g}$. For $\mathfrak{g}=\{0\}$, a quasi-Poisson algebra over $(G, \mathfrak{g})$ is nothing but a Poisson algebra endowed with an action of $G$ by Poisson algebra automorphisms.

2.4. The Lie pair $\left(G_{N}, \mathfrak{g}_{N}\right)$. We will focus in the sequel on the quasi-Poisson algebras over the Lie pair $\left(G_{N}, \mathfrak{g}_{N}\right)$ where $N \geq 1$ is an integer, $G_{N}=\mathrm{GL}_{N}(\mathbb{K})$ is the $N$-th general linear group, and $\mathfrak{g}_{N}=\mathfrak{g l}_{N}(\mathbb{K})$ is the Lie algebra of $N \times N$ matrices with entries in $\mathbb{K}$. The Lie bracket in $\mathfrak{g}_{N}$ is given by $[u, v]=u v-v u$ and $G_{N}$ acts on $\mathfrak{g}_{N}$ by ${ }^{g} v=g v g^{-1}$. For $i, j \in\{1, \ldots, N\}$ let $f_{i j} \in \mathfrak{g}_{N}$ be the elementary matrix whose $(i, j)$-th entry is 1 and all other entries are equal to zero. We consider the tensor

$$
\phi_{N}=-f_{i j} \otimes f_{j k} \otimes f_{k i}+f_{j k} \otimes f_{i j} \otimes f_{k i} \in \mathfrak{g}_{N}^{\otimes 3} .
$$

Here and below we sum up over all repeating indices. The tensor $\phi_{N}$ is skewsymmetric, $G_{N}$-invariant, and $\mathfrak{g}_{N}$-invariant. Indeed, $\phi_{N}$ is the Cartan trivector (2.2.2) determined by the trace pairing $v \cdot w=\operatorname{tr}(v w)$ in $\mathfrak{g}_{N}$. Unless explicitly stated to the contrary, by a quasi-Poisson bracket in a $\left(G_{N}, \mathfrak{g}_{N}\right)$-algebra we will mean a quasi-Poisson bracket associated with $\phi_{N}$.

\section{The algebra $A_{N}$ And the Main theorem}

In this section, we derive from an arbitrary algebra $A$ a sequence of commutative algebras $\left(A_{N}\right)_{N \geq 1}$. Then we apply this construction to the group algebras of the fundamental groups of surfaces and state our main theorem.

3.1. The algebra $A_{N}$. Let $A$ be an algebra. Following $\mathrm{Cb}, \mathrm{VdB}$, we define a sequence of commutative algebras $A_{1}, A_{2}, \ldots$ For $N \geq 1$, the algebra $A_{N}$ is generated by the symbols $a_{i j}$ where $a$ runs over $A$ and $i, j$ run over the set $\{1,2, \ldots, N\}$. These generators commute with each other and satisfy the following relations: $1_{i j}=\delta_{i j}$ where $\delta_{i j}$ is the Kronecker delta; for all $a, b \in A, k \in \mathbb{K}$, and $i, j \in\{1,2, \ldots, N\}$,

$$
(k a)_{i j}=k a_{i j}, \quad(a+b)_{i j}=a_{i j}+b_{i j}, \quad \text { and } \quad(a b)_{i j}=a_{i l} b_{l j} .
$$

(In accordance with our conventions, in the third relation, we sum up over the repeating index l.) The construction of $A_{N}$ is functorial: any algebra homomorphism $f: A \rightarrow A^{\prime}$ induces an algebra homomorphism $f_{N}: A_{N} \rightarrow A_{N}^{\prime}$ by $f_{N}\left(a_{i j}\right)=(f(a))_{i j}$ for all $a \in A$ and $i, j \in\{1, \ldots, N\}$.

The definition of $A_{N}$ is designed so that the functor $\mathcal{A l g} \rightarrow \mathcal{C A l g}, A \mapsto A_{N}$ is left adjoint to the functor $\mathcal{C A l g} \rightarrow \mathcal{A l g}, B \mapsto \operatorname{Mat}_{N}(B)$. Here $\mathcal{A l g}$ and $\mathcal{C} \mathcal{A} l g$ are the categories of algebras and commutative algebras, respectively, and $\operatorname{Mat}_{N}(B)$ is the algebra of $N \times N$-matrices over $B$. In other words, for any commutative algebra $B$, there is a canonical bijection

$$
\operatorname{Hom}_{\mathcal{C A l} l g}\left(A_{N}, B\right) \simeq \operatorname{Hom}_{\mathcal{A l g}}\left(A, \operatorname{Mat}_{N}(B)\right)
$$

which is natural in $A$ and $B$. The bijection (3.1.1) carries any $r: A_{N} \rightarrow B$ to the homomorphism $A \rightarrow \operatorname{Mat}_{N}(B)$ sending any $a \in A$ to the $N \times N$-matrix $\left(r\left(a_{i j}\right)\right)_{i, j}$. The inverse bijection carries any $s: A \rightarrow \operatorname{Mat}_{N}(B)$ to the homomorphism $A_{N} \rightarrow B$ 
sending a generator $a_{i j}$ to the $(i, j)$-th term of the matrix $s(a)$ for all $a \in A$. The existence of a natural isomorphism $\operatorname{Hom}_{\mathcal{C A l g}}\left(A_{N},-\right) \simeq \operatorname{Hom}_{\mathcal{A l g}}\left(A, \operatorname{Mat}_{N}(-)\right)$ can be rephrased in the language of algebraic geometry (see [Do, pp. 17-18] or [Ja, §I.1.3]): there is an affine scheme (over $\mathbb{K})$ whose set of $B$-points is $\operatorname{Hom}_{\mathcal{A l g}}\left(A, \operatorname{Mat}_{N}(B)\right)$ for any commutative algebra $B$, and $A_{N}$ is the coordinate algebra of that scheme.

The linear map $\operatorname{tr}=\operatorname{tr}_{N}: A \rightarrow A_{N}$ carrying any $a \in A$ to $\operatorname{tr}(a)=\sum_{i=1}^{N} a_{i i} \in A_{N}$ is called the trace. It is easy to check that $\operatorname{tr}([A, A])=0$ where $[A, A]$ is the submodule of $A$ spanned by the set $\{a b-b a\}_{a, b \in A}$. Hence the trace induces a linear map $A /[A, A] \rightarrow A_{N}$ also denoted by tr. For any algebra homomorphism $f: A \rightarrow A^{\prime}$, the homomorphism $f_{N}: A_{N} \rightarrow A_{N}^{\prime}$ satisfies $f_{N} \operatorname{tr}=\operatorname{tr} f: A \rightarrow A_{N}^{\prime}$.

The algebra $A_{1}$ corresponding to $N=1$ is the maximal commutative algebra obtained as the quotient of $A$. More precisely, the homomorphism $\operatorname{tr}: A \rightarrow A_{1}$ is surjective and its kernel is the two-sided ideal of $A$ generated by $[A, A]$.

3.2. Actions on $A_{N}$. For all $N \geq 1$, we turn the algebra $A_{N}$ of Section 3.1 into an algebra over the Lie pair $\left(G_{N}, \mathfrak{g}_{N}\right)=\left(\mathrm{GL}_{N}(\mathbb{K}), \mathfrak{g l}_{N}(\mathbb{K})\right)$ introduced in Section 2.4. The action of $G_{N}$ on $A_{N}$ by algebra automorphisms is defined by

$$
g a_{i j}=\left(g^{-1}\right)_{i, k} g_{l, j} a_{k l}
$$

for all $g=\left(g_{k, l}\right)_{k, l=1}^{N} \in G_{N}, a \in A$ and $i, j \in\{1,2, \ldots, N\}$. Though one usually writes numerical coefficients to the left of the variables, as on the right-hand side of (3.2.1), it is easier to remember this formula in the following equivalent form:

$$
g a_{i j}=\left(g^{-1}\right)_{i, k} a_{k l} g_{l, j} .
$$

In the sequel we rather write our formulas in the latter form. The Lie algebra $\mathfrak{g}_{N}$ acts by derivations on the algebra $A_{N}$ by

$$
w a_{i j}=a_{i s} w_{s, j}-w_{i, s} a_{s j}
$$

for all $w=\left(w_{k, l}\right)_{k, l=1}^{N} \in \mathfrak{g}_{N}, a \in A$ and $i, j \in\{1,2, \ldots, N\}$. In terms of the elementary matrices $f_{k l} \in \mathfrak{g}_{N}$ we have $f_{k l} a_{i j}=\delta_{l j} a_{i k}-\delta_{i k} a_{l j}$ for all $i, j, k, l$. Direct computations show that formulas (3.2.2) and (3.2.3) are compatible with the defining relations of $A_{N}$ and turn $A_{N}$ into a $\left(G_{N}, \mathfrak{g}_{N}\right)$-algebra. It is clear that for any algebra homomorphism $A \rightarrow A^{\prime}$, the induced algebra homomorphism $A_{N} \rightarrow A_{N}^{\prime}$ is $\left(G_{N}, \mathfrak{g}_{N}\right)$-equivariant.

The action of $G_{N}$ on $A_{N}$ has the following origin. Given a commutative algebra $B$, the group $G_{N}$ acts on $\operatorname{Mat}_{N}(B)$ by $M \mapsto g M g^{-1}$ for $M \in \operatorname{Mat}_{N}(B)$ and $g \in G_{N}$. This induces a (left) action of $G_{N}$ on $\operatorname{Hom}_{\mathcal{A l g}}\left(A, \operatorname{Mat}_{N}(B)\right)$ and, via (3.1.1), a (left) action of $G_{N}$ on $\operatorname{Hom}_{\mathcal{C A l g}}\left(A_{N}, B\right)$. The latter action is natural in $B$ and therefore, by the Yoneda lemma, it induces a (left) action of $G_{N}$ on $A_{N}$ so that

$$
r(g x)=\left(g^{-1} r\right)(x) \quad \text { for any } r \in \operatorname{Hom}_{\mathcal{C A l g}}\left(A_{N}, B\right), g \in G_{N}, x \in A_{N} .
$$

For $B=A_{N}, r=$ id $: A_{N} \rightarrow B$, and $x=a_{i j}$ with $a \in A$ and $i, j \in\{1, \ldots, N\}$, we obtain $g a_{i j}=\left(g^{-1} \mathrm{id}\right)\left(a_{i j}\right)=\left(g^{-1}\right)_{i, k} a_{k l} g_{l, j}$ as in our definition (3.2.2) of the action of $G_{N}$ on $A_{N}$. The actions of $G_{N}$ and $\mathfrak{g}_{N}$ on $A_{N}$ are further studied in Appendix A.

The algebra $A_{N}$ has three useful subalgebras: $A_{N}^{\mathfrak{g}_{N}}, A_{N}^{G_{N}}$, and the algebra $A_{N}^{t}$ generated by $\operatorname{tr}(A) \subset A_{N}$. It is easy to check that $A_{N}^{t} \subset A_{N}^{\mathfrak{g}_{N}} \cap A_{N}^{G_{N}}$. When $\mathbb{K}$ is a field of characteristic zero and the algebra $A$ is finitely generated, we have $A_{N}^{t}=A_{N}^{G_{N}}$ and therefore $A_{N}^{G_{N}} \subset A_{N}^{\mathfrak{g}_{N}}$; see [LbP, [Cb]. 
3.3. The case of a group algebra. Given a group $\pi$, we can apply the constructions above to the group algebra $A=\mathbb{K} \pi$. This gives for each $N \geq 1$ a commutative $\left(G_{N}, \mathfrak{g}_{N}\right)$-algebra $A_{N}=(\mathbb{K} \pi)_{N}$ generated by the commuting symbols $a_{i j}$ where $a \in \pi$ and $i, j \in\{1, \ldots, N\}$ subject to the relations $1_{i j}=\delta_{i j}$ and $(a b)_{i j}=a_{i l} b_{l j}$ for all $a, b \in \pi$ and $i, j \in\{1, \ldots, N\}$. Note that $A /[A, A]$ is the free $\mathbb{K}$-module with basis $\check{\pi}$ where $\check{\pi}$ is the set of the conjugacy classes of elements of $\pi$. For all $N \geq 1$, we have the linear map $\operatorname{tr}: \mathbb{K} \check{\pi} \rightarrow A_{N}$. The discussion at the end of Section 3.1 shows that $A_{1}$ is the group algebra of $H_{1}(\pi)=\pi /[\pi, \pi]$, and the map $\operatorname{tr}: \mathbb{K} \check{\pi} \rightarrow A_{1}$ is the linear extension of the obvious projection $\check{\pi} \rightarrow H_{1}(\pi)$.

For a commutative algebra $B$, consider the group $\mathrm{GL}_{N}(B)$ of invertible $N \times N$ matrices over $B$ and set $\mathcal{H}_{B}=\operatorname{Hom}\left(\pi, \mathrm{GL}_{N}(B)\right)$. Restricting algebra homomorphisms $A \rightarrow \operatorname{Mat}_{N}(B)$ to $\pi \subset A$, we obtain a bijection

$$
\operatorname{Hom}_{\mathcal{A l g}}\left(A, \operatorname{Mat}_{N}(B)\right) \simeq \operatorname{Hom}\left(\pi, \operatorname{GL}_{N}(B)\right)=\mathcal{H}_{B}
$$

and, composing with (3.1.1), we obtain a bijection

$$
\mathcal{H}_{B} \simeq \operatorname{Hom}_{\mathcal{C A l g}}\left(A_{N}, B\right) .
$$

Thus, there is an affine scheme (over $\mathbb{K}$ ) whose set of $B$-points is $\mathcal{H}_{B}$ for any commutative algebra $B$, and $A_{N}$ is the coordinate algebra of that scheme.

Note that every $x \in A_{N}$ determines a function $\mathcal{H}_{B} \rightarrow B$ which corresponds under (3.3.1) to evaluation at $x$. This defines the evaluation map

$$
\mathrm{ev}_{B}: A_{N} \longrightarrow \operatorname{Map}\left(\mathcal{H}_{B}, B\right),
$$

which is an algebra homomorphism from $A_{N}$ to the algebra $\operatorname{Map}\left(\mathcal{H}_{B}, B\right)$ of $B$ valued functions on $\mathcal{H}_{B}$ with point-wise addition and multiplication. For any $a \in \pi$, $i, j \in\{1, \ldots, N\}$ and $s \in \mathcal{H}_{B}$, we have

$$
\operatorname{ev}_{B}\left(a_{i j}\right)(s)=(i, j) \text {-th term of the matrix } s(a) .
$$

The action of $G_{N}$ on $\mathrm{GL}_{N}(B)$ by conjugations induces an action of $G_{N}$ on $\mathcal{H}_{B}$; the latter induces an action of $G_{N}$ on $\operatorname{Map}\left(\mathcal{H}_{B}, B\right)$ so that $(g f)(h)=f\left(g^{-1} h\right)$ for any $g \in G_{N}, f \in \operatorname{Map}\left(\mathcal{H}_{B}, B\right)$ and $h \in \mathcal{H}_{B}$. The map ev $\operatorname{ev}_{B}$ is $G_{N}$-equivariant: $\operatorname{ev}_{B}(g x)=g \operatorname{ev}_{B}(x)$ for any $x \in A_{N}$ and $g \in G_{N}$. Indeed, for any $s \in \mathcal{H}_{B}$ corresponding through (3.3.1) to $r \in \operatorname{Hom}_{\mathcal{C A l g}}\left(A_{N}, B\right)$, we have

$$
\mathrm{ev}_{B}(g x)(s)=r(g x) \stackrel{\sqrt{3.2 .4}}{=}\left(g^{-1} r\right)(x)=\operatorname{ev}_{B}(x)\left(g^{-1} s\right)=\left(g \operatorname{ev}_{B}(x)\right)(s) .
$$

Consider now the case where $\pi$ is a free group with basis $\left\{x_{u}\right\}_{u \in U}$ indexed by a (possibly infinite) set $U$. It follows from the definitions that the algebra $A_{N}=(\mathbb{K} \pi)_{N}$ is generated by the commuting symbols $x_{i j}^{u}=\left(x_{u}\right)_{i j}$ and $\bar{x}_{i j}^{u}=\left(x_{u}^{-1}\right)_{i j}$ with $u \in U, i, j \in\{1, \ldots, N\}$ subject only to the relation $x_{i l}^{u} \bar{x}_{l j}^{u}=\delta_{i j}$ for all $u, i, j$. These relations may be expressed by saying that the $N \times N$-matrices $x^{u}=\left(x_{i j}^{u}\right)_{i, j}$ and $\bar{x}^{u}=\left(\bar{x}_{i j}^{u}\right)_{i, j}$ are mutually inverse for all $u$. The same algebra is generated by the commuting symbols $y^{u}$ and $x_{i j}^{u}$ with $u \in U$ and $i, j \in\{1, \ldots, N\}$, subject to the relation $y^{u} \operatorname{det}\left(x^{u}\right)=1$ for all $u$. If $U$ is finite and $n=\operatorname{card}(U)$, then $A_{N}$ is the tensor product of $n$ copies of the algebra generated by the commuting symbols $y$ and $x_{i j}$ with $i, j \in\{1, \ldots, N\}$ subject to the single relation $y \operatorname{det}\left(\left(x_{i j}\right)_{i, j}\right)=1$. This is compatible with the standard computation of the coordinate algebra of the affine scheme obtained as the direct product of $n$ copies of $\mathrm{GL}_{N}$ (see, for example, [Do, Example 3.5.6] or [Ja, §I.2.2]). 
3.4. Main theorem. We state our main result. The definition of Goldman's Lie bracket used in this statement will be recalled in Section 7.3.

Theorem 3.1. Let $\Sigma$ be an oriented surface with base point $* \in \partial \Sigma$. Let $\pi=$ $\pi_{1}(\Sigma, *)$ and $A=\mathbb{K} \pi$. Then, for all $N \geq 1$, we have the following:

- the $\left(G_{N}, \mathfrak{g}_{N}\right)$-algebra $A_{N}$ admits a canonical quasi-Poisson bracket $\{-,-\}$ associated with the trivector $\phi_{N}$;

- the trace map $\operatorname{tr}: \mathbb{K} \check{\pi} \rightarrow A_{N}^{\mathfrak{g}_{N}}$ is a homomorphism of Lie algebras where $\mathbb{K} \check{\pi}$ is endowed with $2 \times\left(\right.$ the Goldman Lie bracket) and $A_{N}^{\mathfrak{g}_{N}}$ is endowed with the restriction of the bracket $\{-,-\}$.

The quasi-Poisson bracket in $A_{N}$ produced by this theorem is defined by an explicit formula and does not depend on any provisional choices (see Section 7). This bracket is natural in the sense that it is invariant under homeomorphisms of surfaces preserving orientation and the base point.

The restriction of our quasi-Poisson bracket in $A_{N}$ to $A_{N}^{\mathfrak{g}_{N}}$ is a Poisson bracket turning $A_{N}^{\mathfrak{g}_{N}}$ in a Poisson algebra. The second claim of Theorem 3.1 implies that the subalgebra $A_{N}^{t}$ of $A_{N}^{\mathfrak{g}_{N}}$ generated by $\operatorname{tr}(\check{\pi})$ is a Poisson subalgebra. We may summarize the situation by saying that Goldman's bracket multiplied by 2 induces a Poisson bracket in $A_{N}^{t}$, and the latter extends canonically to a natural quasiPoisson bracket in $A_{N}$. If 2 is invertible in $\mathbb{K}$, then dividing the latter bracket by 2 we obtain a quasi-Poisson bracket in $A_{N}$ which is associated with $\phi_{N} / 4$ and extends the Poisson bracket in $A_{N}^{t}$ induced by Goldman's bracket. If $\mathbb{K}$ is a field of characteristic zero and $\Sigma$ is compact, then $A_{N}^{t}=A_{N}^{G_{N}}$ and we obtain a Poisson bracket in $A_{N}^{G_{N}}$.

For $N=1$ our quasi-Poisson bracket in $A_{1}$, i.e. in the group algebra of $H_{1}(\pi)$, may be computed explicitly by $\{a, b\}=2(a \cdot b) a b$ for all $a, b \in H_{1}(\pi)=H_{1}(\Sigma)$, where - denotes the homological intersection form of $\Sigma$ and we use multiplicative notation for the group operation in $H_{1}(\Sigma)$. This bracket is in fact a Poisson bracket, which is compatible with the equality $A_{1}^{\mathfrak{g}_{1}}=A_{1}$.

The proof of Theorem 3.1 starts with a bilinear form $\eta$ in $A=\mathbb{K} \pi$ defined in $[\mathrm{Tu}$. This form is a Fox pairing in the sense of $[\mathrm{MT}]$. A simple normalization turns $\eta$ into a skew-symmetric Fox pairing $\eta^{s}$ in $A$. We show that every skew-symmetric Fox pairing in $A$ induces a double bracket in $A$ in the sense of Van den Bergh $\mathrm{VdB}$. The double bracket induced by $\eta^{s}$ turns out to be quasi-Poisson. Then a construction of Van den Bergh yields a quasi-Poisson bracket in $A_{N}$. We introduce all these tools in Sections 46 and finish the proof in Section 7 .

\section{Double AND TRIPLE BRACKets}

We outline the theory of "multiple brackets" due to Van den Bergh $[\mathrm{VdB}$. Throughout this section, $A$ is an arbitrary algebra.

4.1. Preliminaries. For $n \geq 2$, we will often write any element $x$ of $A^{\otimes n}$ as $x^{(1)} \otimes \cdots \otimes x^{(n)}$ and drop the summation sign. Given a permutation $\left(i_{1}, \ldots, i_{n}\right)$ of $(1, \ldots, n)$, we denote by $P_{i_{1} \cdots i_{n}}$ the linear map $A^{\otimes n} \rightarrow A^{\otimes n}$ carrying any $x \in A^{\otimes n}$ to $x^{\left(i_{1}\right)} \otimes x^{\left(i_{2}\right)} \otimes \cdots \otimes x^{\left(i_{n}\right)}$. Unless explicitly stated otherwise, we endow $A^{\otimes n}$ with the "outer" $A$-bimodule structure defined by

$$
a x b=a x^{(1)} \otimes x^{(2)} \otimes \cdots \otimes x^{(n-1)} \otimes x^{(n)} b \quad \text { for } a, b \in A \text { and } x \in A^{\otimes n} .
$$

A linear map $D: A \rightarrow A^{\otimes n}$ is a derivation if $D(a b)=a D(b)+D(a) b$ for all $a, b \in A$. 
4.2. Double brackets. A double bracket on the algebra $A$ is a linear map $\{\{-,-\}$ : $A^{\otimes 2} \rightarrow A^{\otimes 2}$ which is a derivation in the second variable and is skew-symmetric. This means that for all $a, b, c \in A$

$$
\left\{\{a, b c\}=b\{\{a, c\}\}+\left\{\{a, b\} c \text { and }\{\{b, a\}\}=-P_{21}\{\{a, b\}\}\right.\right.
$$

These properties imply that $\{\{a b, c\}\}=a *\{\{b, c\}\}+\{a, c\}\} * b$ for all $a, b, c \in A$ where $*$ is the "inner" $A$-bimodule structure on $A^{\otimes 2}$ defined by

$$
l *\left(a_{1} \otimes a_{2}\right) * r=a_{1} r \otimes l a_{2} \quad \text { for } l, a_{1}, a_{2}, r \in A .
$$

We now relate the double brackets to the algebra $A_{N}$ defined in Section 3.1

Lemma 4.1. $\mathrm{VdB}$, Proposition 7.5.1] Given a double bracket $\{\{-,-\}$ in $A$ and an integer $N \geq 1$, there is a unique bilinear form $\{-,-\}: A_{N} \times A_{N} \rightarrow A_{N}$ which is a derivation in each variable and satisfies

$$
\left\{a_{i j}, b_{u v}\right\}=\left\{\{ a , b \} _ { u j } ^ { ( 1 ) } \left\{\{a, b\}_{i v}^{(2)}\right.\right.
$$

for all $a, b \in A$ and $i, j, u, v \in\{1, \ldots, N\}$. This form $\{-,-\}$ is skew-symmetric and satisfies the identities (2.2.3), (2.3.1).

Proof. We extend (4.2.2) to a bilinear form $\{-,-\}: A_{N} \times A_{N} \rightarrow A_{N}$ which is a derivation in each variable. To see that this form is well-defined, we need to verify the compatibility with the defining relations of $A_{N}$. That the right-hand side of (4.2.2) is linear in both $a$ and $b$ follows from the bilinearity of $\{\{-,-\}$. The compatibility with the relation $1_{i j}=\delta_{i j}$ is a consequence of the fact that $\{\{1, b\}=0=\{\{a, 1\}$ for any $a, b \in A$. To verify the compatibility with the relation of type $(b c)_{u v}=b_{u l} c_{l v}$, let us expand $\left\{\{a, b\}=x^{(1)} \otimes x^{(2)}\right.$ and $\{\{a, c\}\}=y^{(1)} \otimes y^{(2)}$. Then

$$
\left\{\{a, b c\}=b\{\{a, c\}\}+\left\{\{a, b\} c=b y^{(1)} \otimes y^{(2)}+x^{(1)} \otimes x^{(2)} c .\right.\right.
$$

Therefore

$$
\begin{aligned}
\left\{a_{i j},(b c)_{u v}\right\} & =\{a, b c\}_{u j}^{(1)}\{a, b c\}_{i v}^{(2)} \\
& =\left(b y^{(1)}\right)_{u j} y_{i v}^{(2)}+x_{u j}^{(1)}\left(x^{(2)} c\right)_{i v} \\
& =b_{u l} y_{l j}^{(1)} y_{i v}^{(2)}+x_{u j}^{(1)} x_{i l}^{(2)} c_{l v} \\
& =b_{u l}\left\{a_{i j}, c_{l v}\right\}+\left\{a_{i j}, b_{u l}\right\} c_{l v}=\left\{a_{i j}, b_{u l} c_{l v}\right\} .
\end{aligned}
$$

A similar computation gives $\left\{(a b)_{i j}, c_{u v}\right\}=\left\{a_{i l} b_{l j}, c_{u v}\right\}$.

The skew-symmetry of $\{-,-\}$ follows from the skew-symmetry of $\{\{-,-\}$ :

$$
\left\{b_{u v}, a_{i j}\right\}=\left\{\{ b , a \} _ { i v } ^ { ( 1 ) } \left\{\{b, a\}_{u j}^{(2)}=-\left\{\{ a , b \} _ { i v } ^ { ( 2 ) } \left\{\{a, b\}_{u j}^{(1)}=-\left\{a_{i j}, b_{u v}\right\} .\right.\right.\right.\right.
$$

Pick $w=\left(w_{k, l}\right)_{k, l} \in \mathfrak{g}_{N}$. It is easy to see that if (2.2.3) holds for the generators of $A_{N}$, then it holds for all elements of $A_{N}$. We check (2.2.3) for the generators:

$$
\begin{aligned}
w\left\{a_{i j}, b_{u v}\right\}= & w\left(\left\{\{a, b\}_{u j}^{(1)}\left\{\{a, b\}_{i v}^{(2)}\right)\right.\right. \\
= & w\left(\{ \{ a , b \} _ { u j } ^ { ( 1 ) } ) \left\{\{a, b\}_{i v}^{(2)}+\{a a, b\}_{u j}^{(1)} w\left(\left\{\{a, b\}_{i v}^{(2)}\right)\right.\right.\right. \\
= & \left\{\{ a , b \} _ { u k } ^ { ( 1 ) } w _ { k , j } \left\{\{a, b\}_{i v}^{(2)}-w_{u, k}\left\{\{ a , b \} _ { k j } ^ { ( 1 ) } \left\{\{a, b\}_{i v}^{(2)}\right.\right.\right.\right. \\
& +\left\{\{ a , b \} _ { u j } ^ { ( 1 ) } \left\{\{a, b\}_{i k}^{(2)} w_{k, v}-\left\{\{ a , b \} _ { u j } ^ { ( 1 ) } w _ { i , k } \left\{\{a, b\}_{k v}^{(2)}\right.\right.\right.\right. \\
= & \left\{a_{i k} w_{k, j}-w_{i, k} a_{k j}, b_{u v}\right\}+\left\{a_{i j}, b_{u k} w_{k, v}-w_{u, k} b_{k v}\right\} \\
= & \left\{w a_{i j}, b_{u v}\right\}+\left\{a_{i j}, w b_{u v}\right\} .
\end{aligned}
$$


Similarly, it is enough to check (2.3.1) for any $g=\left(g_{k, l}\right) \in G_{N}$ and the generators of $A_{N}$. We have

$$
\begin{aligned}
\left\{g a_{i j}, g b_{u v}\right\} & =\left\{\left(g^{-1}\right)_{i, k} a_{k l} g_{l, j},\left(g^{-1}\right)_{u, s} b_{s t} g_{t, v}\right\} \\
& =\left(g^{-1}\right)_{i, k} g_{l, j}\left(g^{-1}\right)_{u, s} g_{t, v}\left\{a_{k l}, b_{s t}\right\} \\
& =\left(g^{-1}\right)_{i, k} g_{l, j}\left(g^{-1}\right)_{u, s} g_{t, v}\left\{\{ a , b \} _ { s l } ^ { ( 1 ) } \left\{\{a, b\}_{k t}^{(2)}\right.\right. \\
& =\left(g^{-1}\right)_{u, s}\left\{\{ a , b \} _ { s l } ^ { ( 1 ) } g _ { l , j } ( g ^ { - 1 } ) _ { i , k } \left\{\{a, b\}_{k t}^{(2)} g_{t, v}\right.\right. \\
& =\left(g \{ \{ a , b \} _ { u j } ^ { ( 1 ) } ) \left(g\left\{\{a, b\}_{i v}^{(2)}\right)\right.\right. \\
& =g\left(\{a, b\}_{u j}^{(1)}\{\{a, b\}\}_{i v}^{(2)}\right)=g\left\{a_{i j}, b_{u v}\right\} .
\end{aligned}
$$

4.3. A bracket $\langle-,-\rangle$. A double bracket $\{\{-,-\}\}$ on $A$ induces further pairings which we now discuss. For $a, b \in A$, set

$$
\langle a, b\rangle=\{\{a, b\}\}^{(1)}\{\{a, b\}\}^{(2)} \in A .
$$

We state several properties of $\langle-,-\rangle: A \times A \rightarrow A$ following [VdB, Section 2.4]:

(i) the composition of $\langle-,-\rangle$ with the projection to $A /[A, A]$ is skew-symmetric;

(ii) $\langle[A, A], A\rangle=0$;

(iii) the pairing $\langle-,-\rangle$ is a derivation in the second variable.

Set $\check{A}=A /[A, A]$. The properties (i) and (ii) imply that $\langle-,-\rangle$ induces bilinear pairings $\check{A} \times A \rightarrow A$ and $\check{A} \times \check{A} \rightarrow \check{A}$. The latter pairing is skew-symmetric. Both pairings are denoted by $\langle-,-\rangle$.

Lemma 4.2. For all $N \geq 1$, the trace map $\operatorname{tr}: \check{A} \rightarrow A_{N}$ carries the bracket $\langle-,-\rangle$ on $\check{A}$ to the bracket $\{-,-\}$ on $A_{N}$ defined by Lemma 4.1 .

Proof. Let $a, b \in A$ and let $\check{a}, \breve{b}$ be their projections to $\check{A}$. We have

$$
\begin{aligned}
\{\operatorname{tr}(\check{a}), \operatorname{tr}(\check{b})\} & =\sum_{i, j}\left\{a_{i i}, b_{j j}\right\} \\
& \stackrel{4.2 .2)}{=} \sum_{i, j}\left\{\{ a , b \} _ { j i } ^ { ( 1 ) } \left\{\{a, b\}_{i j}^{(2)}\right.\right. \\
& =\sum_{j}\left(\left\{\{a, b\}^{(1)}\left\{\{a, b\}^{(2)}\right)_{j j}\right.\right. \\
& =\operatorname{tr}\left(\{\{a, b\}\}^{(1)}\{\{a, b\}\}^{(2)}\right) \stackrel{(4.3 .1)}{=} \operatorname{tr}(\langle a, b\rangle)=\operatorname{tr}(\langle\check{a}, \check{b}\rangle) .
\end{aligned}
$$

4.4. Triple brackets. The brackets $\{-,-\}$ on $A_{N}$ and $\langle-,-\rangle$ on $\check{A}=A /[A, A]$ are skew-symmetric but do not necessarily satisfy the Jacobi identity. Their deviation from the Jacobi identity is formulated in terms of triple brackets on $A$. A triple bracket on $A$ is a linear map $\left\{\{-,-,-\}: A^{\otimes 3} \rightarrow A^{\otimes 3}\right.$ which is a derivation in the third variable and is cyclically symmetric. This means that for all $a, b, c, d \in A$,

$$
\left\{\{a, b, c d\}=c\left\{\{a, b, d\}+\{\{a, b, c\}\} d \text { and } \quad\{c, a, b\}=P_{312}\{\{a, b, c\}\} .\right.\right.
$$

By $\mathrm{VdB}$, Proposition 2.3.1], a double bracket $\{\{-,-\}$ on $A$ determines a triple bracket on $A$ by

$$
\left\{\{-,-,-\}=\sum_{i=0}^{2} P_{312}^{i}\left(\{ \{ - , - \} \otimes \operatorname { i d } _ { A } ) \left(\operatorname{id}_{A} \otimes\{\{-,-\}) P_{312}^{-i} .\right.\right.\right.
$$


Lemma 4.3. $\mathrm{VdB}$, Proposition 7.5.2 \& Corollary 2.4.4] Consider a double bracket $\{\{-,-\}$ on $A$, the associated triple bracket $\{\{-,-,-\}$ on $A$, the induced brackets $\{-,-\}$ on $A_{N}$ for $N \geq 1$ and $\langle-,-\rangle$ on $A$. For any $a, b, c \in A$ and any $p, q, r, s, u, v \in\{1, \ldots, N\}$, we have

$$
\begin{aligned}
& \left\{a_{p q},\left\{b_{r s}, c_{u v}\right\}\right\}+\left\{b_{r s},\left\{c_{u v}, a_{p q}\right\}\right\}+\left\{c_{u v},\left\{a_{p q}, b_{r s}\right\}\right\} \\
= & \left\{\{ a , b , c \} _ { u q } ^ { ( 1 ) } \left\{\{ a , b , c \} _ { p s } ^ { ( 2 ) } \left\{\{a, b, c\}_{r v}^{(3)}-\left\{\{ a , c , b \} _ { r q } ^ { ( 1 ) } \left\{\{ a , c , b \} _ { p v } ^ { ( 2 ) } \left\{\{a, c, b\}_{u s}^{(3)}\right.\right.\right.\right.\right.\right.
\end{aligned}
$$

and

$$
\langle\langle a, b\rangle, c\rangle-\langle a,\langle b, c\rangle\rangle+\langle b,\langle a, c\rangle\rangle=m_{3}(\{[b, a, c\}-\{\{a, b, c\})
$$

where $m_{3}: A^{\otimes 3} \rightarrow A$ carries any $x \in A^{\otimes 3}$ to $x^{(1)} x^{(2)} x^{(3)} \in A$.

A double bracket on $A$ is strong if the associated triple bracket on $A$ satisfies

$$
m_{3}(\{a, b, c\})=m_{3}(\{\{b, a, c\})
$$

for all $a, b, c \in A$. Formula (4.4.3) and properties (i), (ii), (iii) of Section 4.3 imply that if a double bracket on $A$ is strong, then the associated bracket $\langle-,-\rangle$ on $\check{A}$ is a Lie bracket and the pairing $\langle-,-\rangle: \check{A} \times A \rightarrow A$ turns $A$ into a $\check{A}$-algebra. The Lie bracket $\langle-,-\rangle$ in $\check{A}$ determined by a strong double bracket in $A$ has the following property: for each $\check{a} \in \check{A}$, the map $\langle\check{a},-\rangle: \check{A} \rightarrow \check{A}$ is induced by a derivation of $A$. Such a Lie bracket in $\check{A}$ is called by Crawley-Boevey [Cb] an $H_{0}$-Poisson structure on $A$. By [Cb, Theorem 4.5], for any $H_{0}$-Poisson structure $\langle-,-\rangle: \check{A} \times \check{A} \rightarrow \check{A}$ on $A$ there is a unique Poisson bracket $\{-,-\}$ in the subalgebra $A_{N}^{t}$ of $A_{N}$ generated by $\operatorname{tr}(A)$ such that $\{\operatorname{tr}(\check{a}), \operatorname{tr}(\check{b})\}=\operatorname{tr}(\langle\check{a}, \breve{b}\rangle)$ for all $a, b \in A$. When the $H_{0}$-Poisson structure is induced by a strong double bracket on $A$, this claim follows from Lemma 4.2 .

A double bracket on $A$ is Poisson if the associated triple bracket is zero. A Poisson double bracket is strong and moreover, according to (4.4.2), the associated bracket $\{-,-\}$ on $A_{N}$ is a Poisson bracket. We will be interested in a somewhat different class of strong double brackets discussed in the next subsection.

4.5. Quasi-Poisson double brackets. Each derivation $D: A \rightarrow A \otimes A$ determines a triple bracket on $A$ by

$$
\left\{\{a, b, c\}_{D}=D(c)^{(1)} D(a)^{(2)} \otimes D(a)^{(1)} D(b)^{(2)} \otimes D(b)^{(1)} D(c)^{(2)}\right.
$$

for any $a, b, c \in A$. All requirements on a triple bracket are straightforward. (See $\mathrm{VdB}$, Proposition 4.1.1] for a more general construction.) The triple bracket determined in this way by the derivation $E: A \rightarrow A \otimes A$ carrying any $a \in A$ to $a \otimes 1-1 \otimes a$ can be explicitly computed as follows:

$$
\begin{aligned}
\{a, b, c\}_{E}= & a \otimes 1 \otimes b c+1 \otimes a b \otimes c+c a \otimes b \otimes 1+c \otimes a \otimes b \\
& -1 \otimes a \otimes b c-a \otimes b \otimes c-c a \otimes 1 \otimes b-c \otimes a b \otimes 1 .
\end{aligned}
$$

A double bracket $\{\{-,-\}$ on $A$ is quasi-Poisson if the associated triple bracket $\{\{-,-,-\}$ satisfies

$$
\{\{a, b, c\}\}=\left\{\{a, b, c\}_{E}\right.
$$

for all $a, b, c \in A$. It follows from (4.5.1) that a quasi-Poisson double bracket is strong. Hence, it induces a Lie bracket $\langle-,-\rangle$ on $\check{A}$ as well as a $\check{A}$-algebra structure on $A$. 
Lemma 4.4. $\mathrm{VdB}$, Theorem 7.12.2 \& Remark 7.12.3] For any quasi-Poisson double bracket $\left\{\{-,-\}\right.$ on $A$ and any $N \geq 1$, the algebra $A_{N}$ over $\left(G_{N}, \mathfrak{g}_{N}\right)$ with the bracket $\{-,-\}$ provided by Lemma 4.1 is a quasi-Poisson algebra.

Proof. By Lemma 4.1, we need only to verify that for all $x, y, z \in A_{N}$,

$$
\{x,\{y, z\}\}+\{y,\{z, x\}\}+\{z,\{x, y\}\}=\phi_{N}(x, y, z) .
$$

It is straightforward to see that both sides of this formula are skew-symmetric $A_{N^{-}}$ valued trilinear forms on $A_{N}$ which are derivations in each variable. Therefore it suffices to verify (2.2.4) for the generators of $A_{N}$. Let $\mathcal{L}$ and $\mathcal{R}$ be respectively the left-hand and right-hand sides of (4.5.3) for $x=a_{p q}, y=b_{r s}, z=c_{u v}$ with $p, q, r, s, u, v \in\{1, \ldots, N\}$ and $a, b, c \in A$. Computing $\mathcal{L}$ by Lemma 4.3 and then using (4.5.2) and (4.5.1), we obtain that

$$
\begin{aligned}
\mathcal{L}= & a_{u q} \delta_{p s}(b c)_{r v}+\delta_{u q}(a b)_{p s} c_{r v}+(c a)_{u q} b_{p s} \delta_{r v}+c_{u q} a_{p s} b_{r v} \\
& -\delta_{u q} a_{p s}(b c)_{r v}-a_{u q} b_{p s} c_{r v}-(c a)_{u q} \delta_{p s} b_{r v}-c_{u q}(a b)_{p s} \delta_{r v} \\
& -a_{r q} \delta_{p v}(c b)_{u s}-\delta_{r q}(a c)_{p v} b_{u s}-(b a)_{r q} c_{p v} \delta_{u s}-b_{r q} a_{p v} c_{u s} \\
& +\delta_{r q} a_{p v}(c b)_{u s}+a_{r q} c_{p v} b_{u s}+(b a)_{r q} \delta_{p v} c_{u s}+b_{r q}(a c)_{p v} \delta_{u s} .
\end{aligned}
$$

To compute $\mathcal{R}$, we observe that

$$
\begin{aligned}
& \left(f_{i j} \otimes f_{j k} \otimes f_{k i}\right)\left(a_{p q} \otimes b_{r s} \otimes c_{u v}\right) \\
= & \left(\delta_{j q} a_{p i}-\delta_{p i} a_{j q}\right)\left(\delta_{k s} b_{r j}-\delta_{r j} b_{k s}\right)\left(\delta_{i v} c_{u k}-\delta_{u k} c_{i v}\right) \\
= & \delta_{j q} a_{p i} \delta_{k s} b_{r j} \delta_{i v} c_{u k}+\delta_{j q} a_{p i} \delta_{r j} b_{k s} \delta_{u k} c_{i v} \\
& +\delta_{p i} a_{j q} \delta_{k s} b_{r j} \delta_{u k} c_{i v}+\delta_{p i} a_{j q} \delta_{r j} b_{k s} \delta_{i v} c_{u k} \\
& -\delta_{p i} a_{j q} \delta_{k s} b_{r j} \delta_{i v} c_{u k}-\delta_{p i} a_{j q} \delta_{r j} b_{k s} \delta_{u k} c_{i v} \\
& -\delta_{j q} a_{p i} \delta_{k s} b_{r j} \delta_{u k} c_{i v}-\delta_{j q} a_{p i} \delta_{r j} b_{k s} \delta_{i v} c_{u k} \\
= & a_{p v} b_{r q} c_{u s}+a_{p i} \delta_{r q} b_{u s} c_{i v}+a_{j q} b_{r j} \delta_{u s} c_{p v}+a_{r q} b_{k s} \delta_{p v} c_{u k} \\
& -a_{j q} b_{r j} \delta_{p v} c_{u s}-a_{r q} b_{u s} c_{p v}-a_{p i} b_{r q} \delta_{u s} c_{i v}-a_{p v} \delta_{r q} b_{k s} c_{u k} \\
= & a_{p v} b_{r q} c_{u s}+(a c)_{p v} \delta_{r q} b_{u s}+(b a)_{r q} \delta_{u s} c_{p v}+a_{r q}(c b)_{u s} \delta_{p v} \\
& -(b a)_{r q} \delta_{p v} c_{u s}-a_{r q} b_{u s} c_{p v}-(a c)_{p v} b_{r q} \delta_{u s}-a_{p v} \delta_{r q}(c b)_{u s} .
\end{aligned}
$$

Similarly, we have

$$
\begin{aligned}
& \left(f_{j k} \otimes f_{i j} \otimes f_{k i}\right)\left(a_{p q} \otimes b_{r s} \otimes c_{u v}\right) \\
= & \left(\delta_{k q} a_{p j}-\delta_{p j} a_{k q}\right)\left(\delta_{j s} b_{r i}-\delta_{r i} b_{j s}\right)\left(\delta_{i v} c_{u k}-\delta_{u k} c_{i v}\right) \\
= & \delta_{k q} a_{p j} \delta_{j s} b_{r i} \delta_{i v} c_{u k}+\delta_{k q} a_{p j} \delta_{r i} b_{j s} \delta_{u k} c_{i v} \\
& +\delta_{p j} a_{k q} \delta_{j s} b_{r i} \delta_{u k} c_{i v}+\delta_{p j} a_{k q} \delta_{r i} b_{j s} \delta_{i v} c_{u k} \\
& -\delta_{p j} a_{k q} \delta_{j s} b_{r i} \delta_{i v} c_{u k}-\delta_{p j} a_{k q} \delta_{r i} b_{j s} \delta_{u k} c_{i v} \\
& -\delta_{k q} a_{p j} \delta_{j s} b_{r i} \delta_{u k} c_{i v}-\delta_{k q} a_{p j} \delta_{r i} b_{j s} \delta_{i v} c_{u k} \\
= & a_{p s} b_{r v} c_{u q}+a_{p j} b_{j s} \delta_{u q} c_{r v}+a_{u q} \delta_{p s} b_{r i} c_{i v}+a_{k q} b_{p s} \delta_{r v} c_{u k} \\
& -a_{k q} \delta_{p s} b_{r v} c_{u k}-a_{u q} b_{p s} c_{r v}-a_{p s} b_{r i} \delta_{u q} c_{i v}-a_{p j} b_{j s} \delta_{r v} c_{u q} \\
= & a_{p s} b_{r v} c_{u q}+(a b)_{p s} \delta_{u q} c_{r v}+a_{u q} \delta_{p s}(b c)_{r v}+b_{p s} \delta_{r v}(c a)_{u q} \\
& -(c a)_{u q} \delta_{p s} b_{r v}-a_{u q} b_{p s} c_{r v}-a_{p s}(b c)_{r v} \delta_{u q}-(a b)_{p s} \delta_{r v} c_{u q} .
\end{aligned}
$$


Substituting the resulting expressions in the formula

$$
\mathcal{R}=-\left(f_{i j} \otimes f_{j k} \otimes f_{k i}\right)\left(a_{p q} \otimes b_{r s} \otimes c_{u v}\right)+\left(f_{j k} \otimes f_{i j} \otimes f_{k i}\right)\left(a_{p q} \otimes b_{r s} \otimes c_{u v}\right)
$$

we obtain that $\mathcal{R}=\mathcal{L}$.

Under conditions of Lemma 4.4, the $\mathfrak{g}_{N}$-invariant subalgebra $A_{N}^{\mathfrak{g}_{N}}$ of $A_{N}$ is a Poisson algebra, and the trace map $\operatorname{tr}: \check{A} \rightarrow A_{N}^{\mathfrak{g}_{N}}$ is a Lie algebra homomorphism.

4.6. Remark. The original definitions of Van den Bergh apply when $\mathbb{K}$ is a field of characteristic zero; the generalization above to arbitrary commutative rings is straightforward. We slightly modified the definition of a quasi-Poisson double bracket in order to get rid of fractional coefficients appearing in $\mathrm{VdB}$ : a quasiPoisson double bracket in our sense is 2 times a quasi-Poisson double bracket in the sense of $\mathrm{VdB}$.

\section{Fox Derivatives And Fox PAIRINGS}

We introduce and study Fox pairings in augmented algebras.

5.1. Fox derivatives. Fox derivatives were first introduced by Fox in his study of the group rings of free groups. Fox' definitions extend to arbitrary algebras endowed with augmentation homomorphisms. More precisely, let $A$ be an algebra (over $\mathbb{K}$ ) endowed with an algebra homomorphism $\varepsilon: A \rightarrow \mathbb{K}$. A linear map $\partial: A \rightarrow A$ is a left (respectively, a right) Fox derivative if for all $a, b \in A$, we have $\partial(a b)=\partial(a) \varepsilon(b)+a \partial(b)$ (respectively, $\partial(a b)=\partial(a) b+\varepsilon(a) \partial(b))$.

For example, for $e \in A$, the map $A \rightarrow A$ carrying any $a \in A$ to $(a-\varepsilon(a) 1) e$ is a left Fox derivative and the map $A \rightarrow A$ carrying any $a \in A$ to $e(a-\varepsilon(a) 1)$ is a right Fox derivative. Such derivatives are said to be inner.

5.2. Fox pairings. Let $A=(A, \varepsilon)$ be an augmented algebra. A Fox pairing in $A$ or, shorter, an F-pairing in $A$ is a bilinear map $\rho: A \times A \rightarrow A$ which is a left Fox derivative with respect to the first variable and a right Fox derivative with respect to the second variable. In the case of group algebras, an equivalent notion was introduced independently in $\mathrm{Pa}$. under the name of "biderivation" and in $\mathrm{Tu}$ under the name of " $\Delta$-form." Here we follow the terminology of MT.

Note for the record the product formulas

$$
\begin{gathered}
\rho\left(a_{1} a_{2}, b\right)=\rho\left(a_{1}, b\right) \varepsilon\left(a_{2}\right)+a_{1} \rho\left(a_{2}, b\right) \quad \text { for any } a_{1}, a_{2}, b \in A, \\
\rho\left(a, b_{1} b_{2}\right)=\rho\left(a, b_{1}\right) b_{2}+\varepsilon\left(b_{1}\right) \rho\left(a, b_{2}\right) \quad \text { for any } a, b_{1}, b_{2} \in A .
\end{gathered}
$$

For example, any $e \in A$ gives rise to an F-pairing $\rho_{e}$ defined by

$$
\rho_{e}(a, b)=(a-\varepsilon(a) 1) e(b-\varepsilon(b) 1)
$$

for all $a, b \in A$. We call such an F-pairing inner.

Fox pairings in $A$ form a $\mathbb{K}$-module under the usual addition/multiplication by scalars of bilinear forms. The inner F-pairings form a submodule of this module. Two F-pairings $\rho$ and $\rho^{\prime}$ in $A$ are equivalent if they differ by an inner F-pairing, i.e. if there exists $e \in A$ such that $\rho-\rho^{\prime}=\rho_{e}$. 
5.3. Fox pairings in Hopf algebras. Suppose that $A=(A, \Delta, \varepsilon, S)$ is a Hopf algebra with comultiplication $\Delta$, counit $\varepsilon$ and antipode $S$. We shall always use the counit $\varepsilon$ to define F-pairings in $A$. The antipode $S$ induces a transposition on the F-pairings in $A$ as follows. The transpose of a bilinear form $\rho: A \times A \rightarrow A$ is the bilinear form $\bar{\rho}: A \times A \rightarrow A$ defined by

$$
\bar{\rho}(a, b)=S \rho(S(b), S(a))
$$

for $a, b \in A$. For example, the transpose of the inner F-pairing $\rho_{e}$ is $\rho_{S(e)}$ for all $e \in A$. Recall that a Hopf algebra is involutive if its antipode is an involutive map.

Lemma 5.1. If $A$ is involutive, then the transpose $\bar{\rho}$ of any F-pairing $\rho: A \times A \rightarrow A$ is an F-pairing and $\overline{\bar{\rho}}=\rho$.

Proof. For $a_{1}, a_{2}, b \in A$, we have

$$
\begin{aligned}
\bar{\rho}\left(a_{1} a_{2}, b\right) & =S \rho\left(S(b), S\left(a_{1} a_{2}\right)\right) \\
& =S \rho\left(S(b), S\left(a_{2}\right) S\left(a_{1}\right)\right) \\
& =S\left(\rho\left(S(b), S\left(a_{2}\right)\right) S\left(a_{1}\right)+\varepsilon\left(S\left(a_{2}\right)\right) \rho\left(S(b), S\left(a_{1}\right)\right)\right) \\
& =a_{1} S \rho\left(S(b), S\left(a_{2}\right)\right)+\varepsilon\left(a_{2}\right) S \rho\left(S(b), S\left(a_{1}\right)\right) \\
& =a_{1} \bar{\rho}\left(a_{2}, b\right)+\bar{\rho}\left(a_{1}, b\right) \varepsilon\left(a_{2}\right) .
\end{aligned}
$$

A similar computation shows that $\bar{\rho}\left(a, b_{1} b_{2}\right)=\bar{\rho}\left(a, b_{1}\right) b_{2}+\varepsilon\left(b_{1}\right) \bar{\rho}\left(a, b_{2}\right)$ for all $a, b_{1}, b_{2} \in A$. Thus, $\bar{\rho}$ is an F-pairing. For all $a, b \in A$,

$$
\overline{\bar{\rho}}(a, b)=S \bar{\rho}(S(b), S(a))=S^{2} \rho\left(S^{2}(a), S^{2}(b)\right)=\rho(a, b) .
$$

An F-pairing $\rho$ in an involutive Hopf algebra $A$ is skew-symmetric if $\bar{\rho}=-\rho$. Any F-pairing $\rho$ in such an $A$ induces a skew-symmetric F-pairing $\rho^{s}=\rho-\bar{\rho}$ in $A$.

We will need an alternate expression for $\bar{\rho}$, which involves the comultiplication $\Delta$ of $A$. In this expression and in the sequel we use Sweedler's notation for comultiplication: for $a \in A$ we write $\Delta(a)=\sum_{(a)} a^{\prime} \otimes a^{\prime \prime}$ meaning that there is a finite family $\left(a_{i}^{\prime}, a_{i}^{\prime \prime}\right)_{i}$ in $A \times A$ such that $\Delta(a)=\sum_{i} a_{i}^{\prime} \otimes a_{i}^{\prime \prime}$. Similarly, we write

$$
(\Delta \otimes \mathrm{id}) \Delta(a)=(\mathrm{id} \otimes \Delta) \Delta(a)=\sum_{(a)} a^{\prime} \otimes a^{\prime \prime} \otimes a^{\prime \prime \prime} .
$$

Lemma 5.2. If $A$ is involutive, then for any F-pairing $\rho$ in $A$ and any $a, b \in A$, we have $\bar{\rho}(a, b)=\sum_{(a),(b)} a^{\prime} S \rho\left(b^{\prime \prime}, a^{\prime \prime}\right) b^{\prime}$.

Proof. The equality $\sum_{(b)} S\left(b^{\prime}\right) b^{\prime \prime}=\varepsilon(b) 1$ implies that

$$
\begin{aligned}
0=\rho(\varepsilon(b) 1, a) & =\sum_{(b)} \rho\left(S\left(b^{\prime}\right) b^{\prime \prime}, a\right) \\
& =\sum_{(b)}\left(\rho\left(S\left(b^{\prime}\right), a\right) \varepsilon\left(b^{\prime \prime}\right)+S\left(b^{\prime}\right) \rho\left(b^{\prime \prime}, a\right)\right) \\
& =\sum_{(b)}\left(\rho\left(S\left(b^{\prime} \varepsilon\left(b^{\prime \prime}\right)\right), a\right)+S\left(b^{\prime}\right) \rho\left(b^{\prime \prime}, a\right)\right) \\
& =\rho(S(b), a)+\sum_{(b)} S\left(b^{\prime}\right) \rho\left(b^{\prime \prime}, a\right) .
\end{aligned}
$$


Therefore

$$
\rho(S(b), a)=-\sum_{(b)} S\left(b^{\prime}\right) \rho\left(b^{\prime \prime}, a\right) .
$$

A similar computation starting from $\sum_{(a)} S\left(a^{\prime}\right) a^{\prime \prime}=\varepsilon(a) 1$ shows that

$$
\rho(b, a)=-\sum_{(a)} \rho\left(b, S\left(a^{\prime}\right)\right) a^{\prime \prime} .
$$

Replacing here $a$ by $S(a)$ and using the involutivity of $A$, we obtain that

$$
\rho(b, S(a))=-\sum_{(a)} \rho\left(b, a^{\prime \prime}\right) S\left(a^{\prime}\right) .
$$

Now, we can conclude: using (5.3.1) and then (5.3.2), we obtain that

$$
\begin{aligned}
\bar{\rho}(a, b)=S \rho(S(b), S(a)) & =-S\left(\sum_{(b)} S\left(b^{\prime}\right) \rho\left(b^{\prime \prime}, S(a)\right)\right) \\
& =S\left(\sum_{(a),(b)} S\left(b^{\prime}\right) \rho\left(b^{\prime \prime}, a^{\prime \prime}\right) S\left(a^{\prime}\right)\right) \\
& =\sum_{(a),(b)} a^{\prime} S \rho\left(b^{\prime \prime}, a^{\prime \prime}\right) b^{\prime} .
\end{aligned}
$$

\section{From Fox PAirings to Double BRACKets}

In this section, $A=(A, \Delta, \varepsilon, S)$ is a Hopf algebra with comultiplication $\Delta$, counit $\varepsilon$ and antipode $S$. We show, under certain assumptions, how to derive a double bracket in $A$ from a Fox pairing in $A$.

6.1. A double bracket from a Fox pairing. Any bilinear form $\rho: A \times A \rightarrow A$ determines a linear map $\{-,-\}^{\rho}: A \otimes A \rightarrow A \otimes A$ by

$$
\left\{\{a, b\}^{\rho}=\sum_{(a),(b),\left(\rho\left(a^{\prime \prime}, b^{\prime \prime}\right)\right)} b^{\prime} S\left(\left(\rho\left(a^{\prime \prime}, b^{\prime \prime}\right)\right)^{\prime}\right) a^{\prime} \otimes\left(\rho\left(a^{\prime \prime}, b^{\prime \prime}\right)\right)^{\prime \prime}\right.
$$

for any $a, b \in A$.

Lemma 6.1. If $\rho$ is an F-pairing in $A$, then $\left\{[-,-\}^{\rho}\right.$ is a derivation in the second variable.

Proof. For any right Fox derivative $\partial$ in $A$ and any $v \in A$, we define a linear map $\delta=\delta_{v, \partial}: A \rightarrow A \otimes A$ by

$$
\delta(b)=\sum_{(b),\left(\partial\left(b^{\prime \prime}\right)\right)} b^{\prime} S\left(\left(\partial\left(b^{\prime \prime}\right)\right)^{\prime}\right) v \otimes\left(\partial\left(b^{\prime \prime}\right)\right)^{\prime \prime}
$$

for $b \in A$. We claim that this map is a derivation. This will imply the lemma because for any $a \in A$, we have $\{a,-\}^{\rho}=\sum_{(a)} \delta_{a^{\prime}, \rho\left(a^{\prime \prime},-\right)}$.

We now prove the above claim. For any $a, b \in A$, we have

$$
\begin{aligned}
\delta(a b) & =\sum_{(a b),\left(\partial\left((a b)^{\prime \prime}\right)\right)}(a b)^{\prime} S\left(\left(\partial\left((a b)^{\prime \prime}\right)\right)^{\prime}\right) v \otimes\left(\partial\left((a b)^{\prime \prime}\right)\right)^{\prime \prime} \\
& =\sum_{(a),(b),\left(\partial\left(a^{\prime \prime} b^{\prime \prime}\right)\right)} a^{\prime} b^{\prime} S\left(\left(\partial\left(a^{\prime \prime} b^{\prime \prime}\right)\right)^{\prime}\right) v \otimes\left(\partial\left(a^{\prime \prime} b^{\prime \prime}\right)\right)^{\prime \prime} .
\end{aligned}
$$


Using the fact that $\partial\left(a^{\prime \prime} b^{\prime \prime}\right)=\partial\left(a^{\prime \prime}\right) b^{\prime \prime}+\varepsilon\left(a^{\prime \prime}\right) \partial\left(b^{\prime \prime}\right)$, this sum splits into two parts:

$$
\begin{aligned}
& \sum_{(a),(b),\left(\partial\left(a^{\prime \prime}\right) b^{\prime \prime}\right)} a^{\prime} b^{\prime} S\left(\left(\partial\left(a^{\prime \prime}\right) b^{\prime \prime}\right)^{\prime}\right) v \otimes\left(\partial\left(a^{\prime \prime}\right) b^{\prime \prime}\right)^{\prime \prime} \\
= & \sum_{(a),(b),\left(\partial\left(a^{\prime \prime}\right)\right)} a^{\prime} b^{\prime} S\left(\left(\partial\left(a^{\prime \prime}\right)\right)^{\prime} b^{\prime \prime}\right) v \otimes\left(\partial\left(a^{\prime \prime}\right)\right)^{\prime \prime} b^{\prime \prime \prime} \\
= & \sum_{(a),(b),\left(\partial\left(a^{\prime \prime}\right)\right)} a^{\prime} b^{\prime} S\left(b^{\prime \prime}\right) S\left(\left(\partial\left(a^{\prime \prime}\right)\right)^{\prime}\right) v \otimes\left(\partial\left(a^{\prime \prime}\right)\right)^{\prime \prime} b^{\prime \prime \prime} \\
= & \sum_{(a),(b),\left(\partial\left(a^{\prime \prime}\right)\right)} a^{\prime} \varepsilon\left(b^{\prime}\right) S\left(\left(\partial\left(a^{\prime \prime}\right)\right)^{\prime}\right) v \otimes\left(\partial\left(a^{\prime \prime}\right)\right)^{\prime \prime} b^{\prime \prime} \\
= & \sum_{(a),\left(\partial\left(a^{\prime \prime}\right)\right)} a^{\prime} S\left(\left(\partial\left(a^{\prime \prime}\right)\right)^{\prime}\right) v \otimes\left(\partial\left(a^{\prime \prime}\right)\right)^{\prime \prime} b \quad=\delta(a) b
\end{aligned}
$$

and

$$
\begin{aligned}
& \sum_{(a),(b),\left(\varepsilon\left(a^{\prime \prime}\right) \partial\left(b^{\prime \prime}\right)\right)} a^{\prime} b^{\prime} S\left(\left(\varepsilon\left(a^{\prime \prime}\right) \partial\left(b^{\prime \prime}\right)\right)^{\prime}\right) v \otimes\left(\varepsilon\left(a^{\prime \prime}\right) \partial\left(b^{\prime \prime}\right)\right)^{\prime \prime} \\
= & \sum_{(a),(b),\left(\partial\left(b^{\prime \prime}\right)\right)} a^{\prime} \varepsilon\left(a^{\prime \prime}\right) b^{\prime} S\left(\left(\partial\left(b^{\prime \prime}\right)\right)^{\prime}\right) v \otimes\left(\partial\left(b^{\prime \prime}\right)\right)^{\prime \prime} \\
= & \sum_{(b),\left(\partial\left(b^{\prime \prime}\right)\right)} a b^{\prime} S\left(\left(\partial\left(b^{\prime \prime}\right)\right)^{\prime}\right) v \otimes\left(\partial\left(b^{\prime \prime}\right)\right)^{\prime \prime}
\end{aligned}
$$

Lemma 6.2. If $A$ is involutive and $\rho$ is a skew-symmetric F-pairing in $A$, then $\left\{\{-,-\}^{\rho}\right.$ is a double bracket.

Proof. We start by proving that for an arbitrary F-pairing $\rho$ in $A$,

$$
\left\{\{-,-\}^{\bar{\rho}}=P_{21}\left\{\{-,-\}^{\rho} P_{21}\right. \text {. }\right.
$$

By definition, we have

$$
\left\{\{a, b\}^{\bar{\rho}}=\sum_{(a),(b),\left(\bar{\rho}\left(a^{\prime \prime}, b^{\prime \prime}\right)\right)} b^{\prime} S\left(\left(\bar{\rho}\left(a^{\prime \prime}, b^{\prime \prime}\right)\right)^{\prime}\right) a^{\prime} \otimes\left(\bar{\rho}\left(a^{\prime \prime}, b^{\prime \prime}\right)\right)^{\prime \prime}\right.
$$

where, by Lemma 5.2 ,

$$
\bar{\rho}\left(a^{\prime \prime}, b^{\prime \prime}\right)=\sum_{\left(a^{\prime \prime}\right),\left(b^{\prime \prime}\right)}\left(a^{\prime \prime}\right)^{\prime} S \rho\left(\left(b^{\prime \prime}\right)^{\prime \prime},\left(a^{\prime \prime}\right)^{\prime \prime}\right)\left(b^{\prime \prime}\right)^{\prime} .
$$

Therefore

$$
\begin{aligned}
& \{\{a, b\}\}^{\bar{\rho}} \\
& =\sum_{(a),(b),\left(a^{\prime \prime} S \rho\left(b^{\prime \prime \prime}, a^{\prime \prime \prime}\right) b^{\prime \prime}\right)} b^{\prime} S\left(\left(a^{\prime \prime} S \rho\left(b^{\prime \prime \prime}, a^{\prime \prime \prime}\right) b^{\prime \prime}\right)^{\prime}\right) a^{\prime} \otimes\left(a^{\prime \prime} S \rho\left(b^{\prime \prime \prime}, a^{\prime \prime \prime}\right) b^{\prime \prime}\right)^{\prime \prime} \\
& =\sum_{\substack{(a),(b),\left(a^{\prime \prime}\right),\left(b^{\prime \prime}\right) \\
\left(S \rho\left(b^{\prime \prime \prime}, a^{\prime \prime \prime}\right)\right)}} b^{\prime} S\left(\left(a^{\prime \prime}\right)^{\prime}\left(S \rho\left(b^{\prime \prime \prime}, a^{\prime \prime \prime}\right)\right)^{\prime}\left(b^{\prime \prime}\right)^{\prime}\right) a^{\prime} \otimes\left(a^{\prime \prime}\right)^{\prime \prime}\left(S \rho\left(b^{\prime \prime \prime}, a^{\prime \prime \prime}\right)\right)^{\prime \prime}\left(b^{\prime \prime}\right)^{\prime \prime} \\
& =\sum_{(a),(b),\left(S \rho\left(b^{\prime \prime \prime \prime}, a^{\prime \prime \prime \prime}\right)\right)} b^{\prime} S\left(a^{\prime \prime}\left(S \rho\left(b^{\prime \prime \prime \prime}, a^{\prime \prime \prime \prime}\right)\right)^{\prime} b^{\prime \prime}\right) a^{\prime} \otimes a^{\prime \prime \prime}\left(S \rho\left(b^{\prime \prime \prime \prime}, a^{\prime \prime \prime \prime}\right)\right)^{\prime \prime} b^{\prime \prime \prime}
\end{aligned}
$$


QUASI-POISSON STRUCTURES ON REPRESENTATION SPACES OF SURFACES 17

$$
\begin{aligned}
& =\sum_{(a),(b),\left(S \rho\left(b^{\prime \prime \prime \prime}, a^{\prime \prime \prime \prime}\right)\right)} b^{\prime} S\left(b^{\prime \prime}\right) S\left(\left(S \rho\left(b^{\prime \prime \prime \prime}, a^{\prime \prime \prime \prime}\right)\right)^{\prime}\right) S\left(a^{\prime \prime}\right) a^{\prime} \otimes a^{\prime \prime \prime}\left(S \rho\left(b^{\prime \prime \prime \prime}, a^{\prime \prime \prime \prime}\right)\right)^{\prime \prime} b^{\prime \prime \prime} \\
& \left.=\sum_{(a),(b),\left(S \rho\left(b^{\prime \prime \prime}, a^{\prime \prime \prime}\right)\right)} \varepsilon\left(b^{\prime}\right) S\left(\left(S \rho\left(b^{\prime \prime \prime}, a^{\prime \prime \prime}\right)\right)\right)^{\prime}\right) \varepsilon\left(a^{\prime}\right) \otimes a^{\prime \prime}\left(S \rho\left(b^{\prime \prime \prime}, a^{\prime \prime \prime}\right)\right)^{\prime \prime} b^{\prime \prime} \\
& \left.=\quad \sum_{(a),(b),\left(S \rho\left(b^{\prime \prime \prime}, a^{\prime \prime \prime}\right)\right)} S\left(\left(S \rho\left(b^{\prime \prime \prime}, a^{\prime \prime \prime}\right)\right)\right)^{\prime}\right) \otimes \varepsilon\left(a^{\prime}\right) a^{\prime \prime}\left(S \rho\left(b^{\prime \prime \prime}, a^{\prime \prime \prime}\right)\right)^{\prime \prime} \varepsilon\left(b^{\prime}\right) b^{\prime \prime} \\
& =\quad \sum_{(a),(b),\left(S \rho\left(b^{\prime \prime}, a^{\prime \prime}\right)\right)} S\left(\left(S \rho\left(b^{\prime \prime}, a^{\prime \prime}\right)\right)^{\prime}\right) \otimes a^{\prime}\left(S \rho\left(b^{\prime \prime}, a^{\prime \prime}\right)\right)^{\prime \prime} b^{\prime} \\
& =\quad \sum_{(a),(b),\left(\rho\left(b^{\prime \prime}, a^{\prime \prime}\right)\right)} S^{2}\left(\left(\rho\left(b^{\prime \prime}, a^{\prime \prime}\right)\right)^{\prime \prime}\right) \otimes a^{\prime} S\left(\left(\rho\left(b^{\prime \prime}, a^{\prime \prime}\right)\right)^{\prime}\right) b^{\prime}=P_{21}\left(\{b, a\}^{\rho}\right) .
\end{aligned}
$$

If $\rho$ is skew-symmetric, then

$$
\left\{\{-,-\}^{\rho}=-\left\{\{-,-\}^{\bar{\rho}} \stackrel{(6.1 .2)}{=}-P_{21}\left\{\{-,-\}^{\rho} P_{21} .\right.\right.\right.
$$

Now, Lemma 6.1 implies that $\rho$ is a double bracket.

6.2. Computation for inner Fox pairings. The following lemma computes $\left\{[-,-\}^{\rho}: A \otimes A \rightarrow A \otimes A\right.$ for the inner F-pairings $\rho$ in $A$.

Lemma 6.3. If $A$ is involutive, then for any $a, b, e \in A$,

$$
\left\{\{a, b\}^{\rho_{e}}=\sum_{(e)}\left(S\left(e^{\prime}\right) \otimes a e^{\prime \prime} b+b S\left(e^{\prime}\right) a \otimes e^{\prime \prime}-b S\left(e^{\prime}\right) \otimes a e^{\prime \prime}-S\left(e^{\prime}\right) a \otimes e^{\prime \prime} b\right) .\right.
$$

Proof. By definition,

$$
\left\{\{a, b\}^{\rho_{e}}=\sum_{(a),(b),\left(\rho_{e}\left(a^{\prime \prime}, b^{\prime \prime}\right)\right)} b^{\prime} S\left(\left(\rho_{e}\left(a^{\prime \prime}, b^{\prime \prime}\right)\right)^{\prime}\right) a^{\prime} \otimes\left(\rho_{e}\left(a^{\prime \prime}, b^{\prime \prime}\right)\right)^{\prime \prime} .\right.
$$

We have

$$
\begin{aligned}
& \Delta\left(\rho_{e}\left(a^{\prime \prime}, b^{\prime \prime}\right)\right)=\Delta\left(\left(a^{\prime \prime}-\varepsilon\left(a^{\prime \prime}\right) 1\right) \Delta(e) \Delta\left(b^{\prime \prime}-\varepsilon\left(b^{\prime \prime}\right) 1\right)\right. \\
= & \sum_{\left(a^{\prime \prime}\right),(e),\left(b^{\prime \prime}\right)}\left(a^{\prime \prime}\right)^{\prime} e^{\prime}\left(b^{\prime \prime}\right)^{\prime} \otimes\left(a^{\prime \prime}\right)^{\prime \prime} e^{\prime \prime}\left(b^{\prime \prime}\right)^{\prime \prime}+\sum_{(e)} \varepsilon\left(a^{\prime \prime}\right) \varepsilon\left(b^{\prime \prime}\right) e^{\prime} \otimes e^{\prime \prime} \\
& -\sum_{\left(a^{\prime \prime}\right),(e)} \varepsilon\left(b^{\prime \prime}\right)\left(a^{\prime \prime}\right)^{\prime} e^{\prime} \otimes\left(a^{\prime \prime}\right)^{\prime \prime} e^{\prime \prime}-\sum_{(e),\left(b^{\prime \prime}\right)} \varepsilon\left(a^{\prime \prime}\right) e^{\prime}\left(b^{\prime \prime}\right)^{\prime} \otimes e^{\prime \prime}\left(b^{\prime \prime}\right)^{\prime \prime} .
\end{aligned}
$$

Thus,

$$
\{\{a, b\}\}^{\rho_{e}}=X_{a b}+X-X_{a}-X_{b}
$$

where

$$
\begin{aligned}
X_{a b} & =\sum_{(a),(b),\left(a^{\prime \prime}\right),(e),\left(b^{\prime \prime}\right)} b^{\prime} S\left(\left(a^{\prime \prime}\right)^{\prime} e^{\prime}\left(b^{\prime \prime}\right)^{\prime}\right) a^{\prime} \otimes\left(a^{\prime \prime}\right)^{\prime \prime} e^{\prime \prime}\left(b^{\prime \prime}\right)^{\prime \prime} \\
& =\sum_{(a),(b),(e)} b^{\prime} S\left(a^{\prime \prime} e^{\prime} b^{\prime \prime}\right) a^{\prime} \otimes a^{\prime \prime \prime} e^{\prime \prime} b^{\prime \prime \prime} \\
& =\sum_{(a),(b),(e)} b^{\prime} S\left(b^{\prime \prime}\right) S\left(e^{\prime}\right) S\left(a^{\prime \prime}\right) a^{\prime} \otimes a^{\prime \prime \prime} e^{\prime \prime} b^{\prime \prime \prime} \\
& =\sum_{(a),(b),(e)} \varepsilon\left(b^{\prime}\right) S\left(e^{\prime}\right) \varepsilon\left(a^{\prime}\right) \otimes a^{\prime \prime} e^{\prime \prime} b^{\prime \prime}=\sum_{(e)} S\left(e^{\prime}\right) \otimes a e^{\prime \prime} b,
\end{aligned}
$$




$$
\begin{aligned}
X_{a} & =\sum_{(a),(b),\left(a^{\prime \prime}\right),(e)} \varepsilon\left(b^{\prime \prime}\right) b^{\prime} S\left(\left(a^{\prime \prime}\right)^{\prime} e^{\prime}\right) a^{\prime} \otimes\left(a^{\prime \prime}\right)^{\prime \prime} e^{\prime \prime} \\
& =\sum_{(a),(e)} b S\left(a^{\prime \prime} e^{\prime}\right) a^{\prime} \otimes a^{\prime \prime \prime} e^{\prime \prime} \\
& =\sum_{(a),(e)} b S\left(e^{\prime}\right) S\left(a^{\prime \prime}\right) a^{\prime} \otimes a^{\prime \prime \prime} e^{\prime \prime} \\
& =\sum_{(a),(e)} b S\left(e^{\prime}\right) \varepsilon\left(a^{\prime}\right) \otimes a^{\prime \prime} e^{\prime \prime}=\sum_{(e)} b S\left(e^{\prime}\right) \otimes a e^{\prime \prime}, \\
X_{b} & =\sum_{(a),(b),(e),\left(b^{\prime \prime}\right)} b^{\prime} S\left(e^{\prime}\left(b^{\prime \prime}\right)^{\prime}\right) \varepsilon\left(a^{\prime \prime}\right) a^{\prime} \otimes e^{\prime \prime}\left(b^{\prime \prime}\right)^{\prime \prime} \\
& =\sum_{(b),(e)} b^{\prime} S\left(e^{\prime} b^{\prime \prime}\right) a \otimes e^{\prime \prime} b^{\prime \prime \prime} \\
& =\sum_{(b),(e)} b^{\prime} S\left(b^{\prime \prime}\right) S\left(e^{\prime}\right) a \otimes e^{\prime \prime} b^{\prime \prime \prime} \\
& =\sum_{(b),(e)} \varepsilon\left(b^{\prime}\right) S\left(e^{\prime}\right) a \otimes e^{\prime \prime} b^{\prime \prime}=\sum_{(e)} S\left(e^{\prime}\right) a \otimes e^{\prime \prime} b
\end{aligned}
$$

and

$$
X=\sum_{(a),(b),(e)} \varepsilon\left(b^{\prime \prime}\right) b^{\prime} S\left(e^{\prime}\right) \varepsilon\left(a^{\prime \prime}\right) a^{\prime} \otimes e^{\prime \prime}=\sum_{(e)} b S\left(e^{\prime}\right) a \otimes e^{\prime \prime} .
$$

6.3. Remarks. 1. If $\{\{-,-\}$ is a double bracket in an augmented algebra $A=$ $(A, \varepsilon)$, then the map $A \times A \rightarrow A$ carrying any pair $(a, b) \in A \times A$ to $\left(\varepsilon \otimes \operatorname{id}_{A}\right)\{\{a, b\}$ is an F-pairing. This defines a map, $\Phi$, from the set of double brackets in $A$ to the set of F-pairings in $A$. If $A$ is an involutive Hopf algebra, then Lemma 6.2 defines a map, $\Psi$, from the set of skew-symmetric F-pairings in $A$ to set of double brackets in $A$. Clearly, $\Phi \Psi=$ id. Generally speaking, the composition $\Psi \Phi$ is not defined.

2. As indicated to the authors by P. Schauenburg, the fact that a right Fox derivative in a Hopf algebra $A$ and an element of $A$ determine a derivation $A \rightarrow$ $A \otimes A$ by (6.1.1) can be alternatively explained in terms of $A$-bimodules. The explanation is based on the following two facts: 1 ) derivations $A \rightarrow M$ with values in an $A$-bimodule $M$ bijectively correspond to $A$-bimodule maps from the $A$-bimodule of differentials on $A$ to $M$, and 2) the Hopf algebra structure on $A$ allows to form the tensor product of $A$-bimodules.

\section{The HOMOTOPy INTERSECTION FORM OF A SURFACE}

In this section, $\Sigma$ is an oriented surface with non-empty boundary and a base point $* \in \partial \Sigma$. We set $\pi=\pi_{1}(\Sigma, *)$ and consider the group algebra $A=\mathbb{K} \pi$.

7.1. The pairing $\eta$. We provide $\partial \Sigma$ with the orientation induced by that of $\Sigma$. By paths and loops we shall mean piecewise-smooth paths and loops in $\Sigma$. The product $\alpha \beta$ of two paths $\alpha$ and $\beta$ is obtained by running first along $\alpha$ and then along $\beta$. Given two distinct simple (that is, multiplicity 1 ) points $p, q$ on a path $\alpha$, we denote by $\alpha_{p q}$ the path from $p$ to $q$ running along $\alpha$ in the positive direction.

We shall use a second base point $\bullet \in \partial \Sigma \backslash\{*\}$ lying "slightly before" $*$ on $\partial \Sigma$. We fix an embedded path $\nu_{\bullet *}$ running from $\bullet$ to $*$ along $\partial \Sigma$ in the positive direction, 
and we denote the inverse path by $\bar{\nu}_{* \bullet}$. The element of $\pi$ represented by a loop $\alpha$ based at $*$ is denoted $[\alpha]$. We say that a loop $\alpha$ based at $\bullet$ represents $\left[\bar{\nu}_{* \bullet} \alpha \nu_{\bullet *}\right] \in \pi$.

The homotopy intersection form of $\Sigma$ is the bilinear map $\eta: A \times A \rightarrow A$ defined, for any $a, b \in \pi$, by

$$
\eta(a, b)=\sum_{p \in \alpha \cap \beta} \varepsilon_{p}(\alpha, \beta)\left[\bar{\nu}_{* \bullet} \alpha \bullet p \beta_{p *}\right] .
$$

Here, $\alpha$ is a loop based at $\bullet$ and representing $a, \beta$ is a loop based at $*$ and representing $b$ so that $\alpha$ and $\beta$ meet transversely in a finite set $\alpha \cap \beta$ of simple points of $\alpha, \beta$. Each crossing $p \in \alpha \cap \beta$ has a sign $\varepsilon_{p}(\alpha, \beta)= \pm 1$ which is equal to +1 if the frame (the positive tangent vector of $\alpha$ at $p$, the positive tangent vector of $\beta$ at $p$ ) is positively oriented and is equal to -1 otherwise. It is easy to verify that $\eta$ is a well-defined F-pairing: this is essentially the intersection pairing introduced in $\mathrm{Tu}$, see also $[\mathrm{Pe}$ and $[\mathrm{MT}]$. The pairing $\eta$ is connected to Reidemeister's equivariant intersection pairings and, in this form, it appears implicitly in [Pa].

7.2. The pairing $\eta^{s}$. The group algebra $A=\mathbb{K} \pi$ has a canonical structure of an involutive Hopf algebra. The comultiplication $\Delta: A \rightarrow A \otimes A$, the counit $\varepsilon: A \rightarrow \mathbb{K}$ and the antipode $S: A \rightarrow A$ are defined by

$$
\Delta(a)=a \otimes a, \quad \varepsilon(a)=1, \quad S(a)=a^{-1} \quad \text { for any } \quad a \in \pi \subset A .
$$

By Lemma [5.2, the transpose $\bar{\eta}: A \times A \rightarrow A$ of $\eta$ is given by $\bar{\eta}(a, b)=a S(\eta(b, a)) b$ for $a, b \in \pi$. It is easy to check (see [Tu], $\left[\mathrm{MT}\right.$ ) that $\eta+\bar{\eta}=-\rho_{1}$ where $\rho_{1}$ is the inner F-pairing in $A$ associated with $1 \in A$. The F-pairing

$$
\eta^{s}=\eta-\bar{\eta}=2 \eta+\rho_{1}: A \times A \rightarrow A
$$

is skew-symmetric. By definition, for any $a, b \in A$,

$$
\eta^{s}(a, b)=2 \eta(a, b)+(a-\varepsilon(a) 1)(b-\varepsilon(b) 1) .
$$

Let $\{\{-,-\}\}^{s}=\{\{-,-\}\}^{s}$ be the double bracket on $A$ determined by $\eta^{s}$. Lemma 6.3 and formula (7.2.1) give, for all $a, b \in A$,

$$
\left\{\{a, b\}^{s}=2\left\{\{a, b\}^{\eta}+1 \otimes a b+b a \otimes 1-a \otimes b-b \otimes a .\right.\right.
$$

For $a, b \in \pi$, the term $\{a a, b\}\}^{\eta}$ can be explicitly computed as follows.

Lemma 7.1. For any $a, b \in \pi$,

$$
\left\{\{a, b\}^{\eta}=\sum_{p \in \alpha \cap \beta} \varepsilon_{p}(\alpha, \beta)\left[\beta_{* p} \alpha_{p \bullet} \nu_{\bullet *}\right] \otimes\left[\bar{\nu}_{* \bullet} \alpha_{\bullet} \beta_{p *}\right]\right.
$$

where $\alpha$ is a loop based at $\bullet$ and representing $a, \beta$ is a loop based at $*$ and representing $b$ such that $\alpha$ and $\beta$ meet transversely in a finite set of simple points.

Proof. Since $a$ and $b$ are group-like elements of $A$, we have

$$
\left\{\{a, b\}^{\eta}=\sum_{(\eta(a, b))} b S\left(\eta(a, b)^{\prime}\right) a \otimes \eta(a, b)^{\prime \prime} .\right.
$$

Applying (7.1.1) we obtain that

$$
\begin{aligned}
& \left\{\{a, b\}^{\eta}=\sum_{p \in \alpha \cap \beta} \varepsilon_{p}(\alpha, \beta) b S\left(\left[\bar{\nu}_{* \bullet} \alpha_{\bullet p} \beta_{p *}\right]\right) a \otimes\left[\bar{\nu}_{* \bullet} \alpha_{\bullet p} \beta_{p *}\right]\right. \\
& =\sum_{p \in \alpha \cap \beta} \varepsilon_{p}(\alpha, \beta)[\beta]\left[\left(\beta^{-1}\right)_{* p}\left(\alpha^{-1}\right)_{p \bullet} \nu_{\bullet *}\right]\left[\bar{\nu}_{* \bullet} \alpha \nu_{\bullet *}\right] \otimes\left[\bar{\nu}_{* \bullet} \alpha \bullet p \beta\right.
\end{aligned}
$$




$$
\begin{aligned}
& =\sum_{p \in \alpha \cap \beta} \varepsilon_{p}(\alpha, \beta)\left[\beta_{* p}\left(\alpha^{-1}\right)_{p \bullet} \alpha \nu_{\bullet *}\right] \otimes\left[\bar{\nu}_{* \bullet} \alpha{ }_{\bullet} \beta_{p *}\right] \\
& =\sum_{p \in \alpha \cap \beta} \varepsilon_{p}(\alpha, \beta)\left[\beta_{* p} \alpha_{p \bullet \nu_{\bullet *}}\right] \otimes\left[\bar{\nu}_{* \bullet} \alpha_{\bullet} \beta_{p *}\right] .
\end{aligned}
$$

Lemma 7.2. The double bracket $\{\{-,-\}\}^{s}$ is quasi-Poisson.

Proof. In the proof we will use the following notation suggested in $\mathrm{VdB}$. Given a map $\{\{-,-\}\}: A^{\otimes 2} \rightarrow A^{\otimes 2}$ and $u \in A, v \in A^{\otimes 2}$, we set

$$
\begin{aligned}
& \left\{\{u, v\}_{L}=\left\{\left\{u, v^{(1)}\right\}\right\} \otimes v^{(2)}, \quad\left\{\{u, v\}_{R}=v^{(1)} \otimes\left\{\left\{u, v^{(2)}\right\}\right\},\right.\right. \\
& \left\{\{v, u\}_{L}=\left\{\left\{v^{(1)}, u\right\}\right\} \otimes v^{(2)}, \quad\left\{\{v, u\}_{R}=v^{(1)} \otimes\left\{\left\{v^{(2)}, u\right\}\right\} .\right.\right.
\end{aligned}
$$

Let $\left\{\{-,-,-\}^{s}\right.$ be the triple bracket in $A$ determined by $\left\{\{-,-\}^{s}\right.$. We must prove that for all $a, b, c \in A$,

$$
\begin{aligned}
\{a, b, c\}^{s}= & a \otimes 1 \otimes b c+1 \otimes a b \otimes c+c a \otimes b \otimes 1+c \otimes a \otimes b \\
& -1 \otimes a \otimes b c-a \otimes b \otimes c-c a \otimes 1 \otimes b-c \otimes a b \otimes 1 .
\end{aligned}
$$

It is enough to consider $a, b, c \in \pi$. Using the skew-symmetry of $\left\{\{-,-\}^{s}\right.$, we obtain

$$
\begin{aligned}
& \{\{a, b, c\}\}^{s}=\left\{\left\{a,\{\{b, c\}\}^{s}\right\}_{L}^{s}+P_{312}^{-1}\left\{\left\{c,\{\{a, b\}\}^{s}\right\}_{L}^{s}+P_{312}\left\{\left\{b,\{\{c, a\}\}^{s}\right\}\right\}_{L}^{s}\right.\right. \\
& =\left\{\left\{a,\{b, c\}^{s}\right\}_{L}^{s}-P_{312}^{-1} P_{213}\left\{\left\{\{a, b\}^{s}, c\right\}_{L}^{s}-P_{312}\left\{a b, P_{21}\{\{a, c\}\}^{s}\right\}_{L}^{s}\right.\right. \\
& =\left\{\left\{a,\{\{b, c\}\}^{s}\right\}_{L}^{s}-P_{132}\left\{\{\{a, b\}\}^{s}, c\right\}_{L}^{s}-\left\{\left\{b,\{\{a, c)\}^{s}\right\}_{R}^{s}\right. \text {. }\right.
\end{aligned}
$$

We now compute the three resulting terms. Firstly, we have

$$
\left\{\left\{a,\{\{b, c\}\}^{s}\right\}_{L}^{s}=2\left\{\left\{a,\{\{b, c\}\}^{\eta}\right\}_{L}^{s}+\{\{a, 1 \otimes b c+c b \otimes 1-c \otimes b-b \otimes c\}\}_{L}^{s},\right.\right.
$$

where

$$
\begin{aligned}
2\left\{\left\{a,\{\{b, c\}\}^{\eta}\right\}_{L}^{s}=\right. & 4\left\{\left\{a,\{\{b, c\}\}^{\eta}\right\}\right\}_{L}^{\eta}+2 \otimes a\{\{b, c\}\}^{\eta}-2 a \otimes\{\{b, c\}\}^{\eta} \\
& +2 P_{132}\left(\{\{b, c\}\}^{\eta} * a \otimes 1\right)-2 P_{213}\left(a \otimes\{\{b, c\}\}^{\eta}\right)
\end{aligned}
$$

and

$$
\begin{aligned}
& \{a, 1 \otimes b c+c b \otimes 1-c \otimes b-b \otimes c\}_{L}^{s} \\
= & c\left\{\{a, b\}^{s} \otimes 1+\{a, c\}\right\}^{s} b \otimes 1-\{\{a, c\}\}^{s} \otimes b-\{\{a, b\}\}^{s} \otimes c \\
= & 2 c\left\{\{a, b\}^{\eta} \otimes 1+2\{\{a, c\}\}^{\eta} b \otimes 1-2\{\{a, c\}\}^{\eta} \otimes b-2\{a, b\}\right\}^{\eta} \otimes c \\
& +c \otimes a b \otimes 1+c b a \otimes 1 \otimes 1-c a \otimes b \otimes 1-c b \otimes a \otimes 1 \\
& +1 \otimes a c b \otimes 1+c a \otimes b \otimes 1-a \otimes c b \otimes 1-c \otimes a b \otimes 1 \\
& -1 \otimes a c \otimes b-c a \otimes 1 \otimes b+a \otimes c \otimes b+c \otimes a \otimes b \\
& -1 \otimes a b \otimes c-b a \otimes 1 \otimes c+a \otimes b \otimes c+b \otimes a \otimes c .
\end{aligned}
$$

Secondly, we have

$$
\begin{aligned}
& \left.-P_{132}\left\{\{\{a, b\}\}^{s}, c\right\}\right\}_{L}^{s} \\
= & -2 P_{132}\left\{\left\{\{a, b\}^{\eta}, c\right\}_{L}^{s}-P_{132}\{11 \otimes a b+b a \otimes 1-b \otimes a-a \otimes b, c\}_{L}^{s}\right.
\end{aligned}
$$

where

$$
\begin{aligned}
(7.2 .8) & -2 P_{132}\left\{\{\{a, b\}\}^{\eta}, c\right\}_{L}^{s} \\
= & -4 P_{132}\left\{\{\{a, b\}\}^{\eta}, c\right\}_{L}^{\eta}-2 P_{132} P_{132}\left(c\{\{a, b\}\}^{\eta} \otimes 1\right)-2 P_{132}\left(1 \otimes\{\{a, b\}\}^{\eta} * c\right) \\
& \left.+2 P_{132} P_{132}\left(\{\{a, b\}\}^{\eta} \otimes c\right)+2 P_{132}(c \otimes\{a, b\}\}^{\eta}\right)
\end{aligned}
$$




$$
\begin{aligned}
= & -4 P_{132}\left\{\left\{\{a a, b\}^{\eta}, c\right\}_{L}^{\eta}-2 c\{\{a, b\}\}^{\eta} \otimes 1-2 P_{132}\left(1 \otimes\{\{a, b\}\}^{\eta} * c\right)\right. \\
& \left.+2\{\{a, b\}\}^{\eta} \otimes c+2 P_{132}(c \otimes\{a, b\}\}^{\eta}\right)
\end{aligned}
$$

and

$$
\begin{aligned}
& -P_{132}\left\{\{1 \otimes a b+b a \otimes 1-b \otimes a-a \otimes b, c\}_{L}^{s}\right. \\
= & -P_{132}\left(b *\left\{\{a, c\}^{s} \otimes 1\right)-P_{132}\left(\{\{b, c)\}^{s} * a \otimes 1\right)\right. \\
& +P_{132}\left(\{\{b, c\}\}^{s} \otimes a\right)+P_{132}\left(\{\{a, c\}\}^{s} \otimes b\right) \\
= & -2 P_{132}\left(b *\{\{a, c\}\}^{\eta} \otimes 1\right)-2 P_{132}\left(\left\{\{b, c\}^{\eta} * a \otimes 1\right)\right. \\
& +2 P_{132}\left(\left\{\{b, c\}^{\eta} \otimes a\right)+2 P_{132}\left(\{\{a, c\}\}^{\eta} \otimes b\right)\right. \\
& -1 \otimes 1 \otimes b a c-c a \otimes 1 \otimes b+a \otimes 1 \otimes b c+c \otimes 1 \otimes b a \\
& -c b a \otimes 1 \otimes 1-a \otimes 1 \otimes b c+b a \otimes 1 \otimes c+c a \otimes 1 \otimes b \\
& +c b \otimes a \otimes 1+1 \otimes a \otimes b c-b \otimes a \otimes c-c \otimes a \otimes b \\
& +c a \otimes b \otimes 1+1 \otimes b \otimes a c-a \otimes b \otimes c-c \otimes b \otimes a .
\end{aligned}
$$

Thirdly, we have

$$
-\left\{\{b,\{a, c\}\}^{s}\right\}_{R}^{s}=-2\left\{\left\{b,\{\{a, c\}\}^{\eta}\right\}\right\}_{R}^{s}-\{\{b, 1 \otimes a c+c a \otimes 1-c \otimes a-a \otimes c\}\}_{R}^{s}
$$

where

$$
\begin{aligned}
(7.2 .10)-2\left\{\left\{b,\{\{a, c)\}^{\eta}\right\}_{R}^{s}=\right. & -4\left\{\left[b,\{\{a, c\}\}^{\eta}\right\}\right\}_{R}^{\eta}-2\{\{a, c\}\}^{\eta} b \otimes 1+2\left\{\{a, c\}^{\eta} \otimes b\right. \\
& \left.-2 P_{132}\left(b *\{\{a, c\}\}^{\eta} \otimes 1\right)+2 P_{132}(\{a a, c\}\}^{\eta} \otimes b\right)
\end{aligned}
$$

and

$$
\begin{aligned}
& -\left\{\{b, 1 \otimes a c+c a \otimes 1-c \otimes a-a \otimes c\}_{R}^{s}\right. \\
= & \left.-1 \otimes a\{\{b, c\}\}^{s}-1 \otimes\{\{b, a\}\}^{s} c+c \otimes\{\{b, a\}\}^{s}+a \otimes\{b, c\}\right\}^{s} \\
= & \left.-2 \otimes a\{\{b, c\}\}^{\eta}-2 \otimes\{b b, a\}\right\}^{\eta} c+2 c \otimes\{\{b, a\}\}^{\eta}+2 a \otimes\{\{b, c\}\}^{\eta} \\
& -1 \otimes a \otimes b c-1 \otimes a c b \otimes 1+1 \otimes a b \otimes c+1 \otimes a c \otimes b \\
& -1 \otimes a b \otimes c-1 \otimes 1 \otimes b a c+1 \otimes b \otimes a c+1 \otimes a \otimes b c \\
& +c \otimes a b \otimes 1+c \otimes 1 \otimes b a-c \otimes b \otimes a-c \otimes a \otimes b \\
& +a \otimes c b \otimes 1+a \otimes 1 \otimes b c-a \otimes b \otimes c-a \otimes c \otimes b .
\end{aligned}
$$

Summing up the equalities (7.2.6) - (7.2.11) and canceling identical terms with opposite signs, we obtain that $\{a a, b, c\}\}^{s}$ is equal to the following sum:

$$
\begin{aligned}
& 4\left\{\left\{a,\{\{b, c\}\}^{\eta}\right\}_{L}^{\eta}-4 P_{132}\left\{\left\{\{a, b\}^{\eta}, c\right\}\right\}_{L}^{\eta}-4\left\{\left\{b,\{\{a, c\}\}^{\eta}\right\}_{R}^{\eta}\right.\right. \\
& -4 P_{132}\left(b *\{\{a, c\}\}^{\eta} \otimes 1\right)+4 P_{132}\left(\{\{a, c\}\}^{\eta} \otimes b\right)-2 P_{132}\left(1 \otimes\{\{a, b\}\}^{\eta} * c\right) \\
& -2 \otimes\left\{\{b, a\}^{\eta} c+2 P_{132}\left(c \otimes\{\{a, b\}\}^{\eta}\right)+2 c \otimes\{\{b, a\}\}^{\eta}\right. \\
& -2 \otimes 1 \otimes b a c+2 c \otimes 1 \otimes b a+2 \otimes b \otimes a c-2 c \otimes b \otimes a \\
& -c a \otimes 1 \otimes b+c a \otimes b \otimes 1-1 \otimes a b \otimes c+1 \otimes a \otimes b c \\
& +a \otimes 1 \otimes b c+c \otimes a b \otimes 1-c \otimes a \otimes b-a \otimes b \otimes c .
\end{aligned}
$$

Next, we deduce from the equality $\eta+\bar{\eta}=-\rho_{1}$ and (6.1.2) that

$$
-P_{21}\{\{a, b\}\}^{\eta}=\{\{b, a\}\}^{\eta}+a b \otimes 1+1 \otimes b a-a \otimes b-b \otimes a .
$$

Hence

$$
\begin{aligned}
& -2 P_{132}\left(1 \otimes\left\{\{a, b\}^{\eta} * c\right)=-2 \otimes P_{21}\left(\{\{a, b\}\}^{\eta}\right) c\right. \\
= & 2 \otimes\{\{b, a\}\}^{\eta} c+2 \otimes a b \otimes c+2 \otimes 1 \otimes b a c-2 \otimes a \otimes b c-2 \otimes b \otimes a c
\end{aligned}
$$


and

$$
\begin{aligned}
& 2 P_{132}\left(c \otimes\{\{a, b\}\}^{\eta}\right)=2 c \otimes P_{21}\left(\{\{a, b\}\}^{\eta}\right) \\
= & -2 c \otimes\{\{b, a\}\}^{\eta}-2 c \otimes a b \otimes 1-2 c \otimes 1 \otimes b a+2 c \otimes a \otimes b+2 c \otimes b \otimes a .
\end{aligned}
$$

Thus, the expression for $\left\{\{a, b, c\}^{s}\right.$ above simplifies to

$$
\begin{aligned}
\left\{\{a, b, c\}^{s}=\right. & 4\left\{\left\{a,\left\{\{b, c\}^{\eta}\right\}_{L}^{\eta}-4 P_{132}\left\{\{\{a, b\}\}^{\eta}, c\right\}_{L}^{\eta}-4\left\{\left\{b,\left\{\{a, c\}^{\eta}\right\}_{R}^{\eta}\right.\right.\right.\right. \\
& -4 P_{132}\left(b *\left\{\{a, c\}^{\eta} \otimes 1\right)+4 P_{132}\left(\{\{a, c\}\}^{\eta} \otimes b\right)\right. \\
& +a \otimes 1 \otimes b c+1 \otimes a b \otimes c+c a \otimes b \otimes 1+c \otimes a \otimes b \\
& -1 \otimes a \otimes b c-a \otimes b \otimes c-c a \otimes 1 \otimes b-c \otimes a b \otimes 1 .
\end{aligned}
$$

To finish the proof of (7.2.5), it suffices to show that

$$
\begin{aligned}
& \left.\left\{\{a,\{\{b, c\}\}\}^{\eta}\right\}\right\}_{L}^{\eta}-P_{132}\left\{\left\{\{\{a, b\}\}^{\eta}, c\right\}\right\}_{L}^{\eta}-\left\{\left\{b,\{\{a, c\}\}^{\eta}\right\}\right\}_{R}^{\eta} \\
= & P_{132}\left(b *\{\{a, c\}\}^{\eta} \otimes 1\right)-P_{132}\left(\{\{a, c\}\}^{\eta} \otimes b\right) .
\end{aligned}
$$

To proceed, we fix a third base point $\circ \in \partial \Sigma$ which we assume to be "slightly before" - on $\partial \Sigma$. Consider a loop $\alpha$ based at $\circ$ representing $a$ (i.e. $\left[\bar{\nu}_{* \circ} \alpha \nu_{\circ *}\right]=$ $a \in \pi$ ), a loop $\beta$ based at $\bullet$ representing $b$, and a loop $\gamma$ based at $*$ representing $c$; we assume that $\alpha, \beta, \gamma$ meet transversely in a finite set of simple points and that $\alpha \cap \beta \cap \gamma=\varnothing$. The first term in (7.2.12) is

$$
\begin{aligned}
& \left\{\left\{a,\{\{b, c\}\}^{\eta}\right\}_{L}^{\eta}=\sum_{p \in \beta \cap \gamma} \varepsilon_{p}(\beta, \gamma)\left\{\left\{a,\left[\gamma_{* p} \beta_{p \bullet \nu_{\bullet}}\right] \otimes\left[\bar{\nu}_{* \bullet} \beta \bullet p \gamma_{p *}\right]\right\}_{L}^{\eta}\right.\right. \\
= & \sum_{p \in \beta \cap \gamma} \varepsilon_{p}(\beta, \gamma)\left\{\left\{a,\left[\gamma_{* p} \beta_{p \bullet \nu_{\bullet *}}\right]\right\}^{\eta} \otimes\left[\bar{\nu}_{* \bullet} \beta \bullet p \gamma_{p *}\right]\right. \\
= & \sum_{\substack{p \in \beta \cap \gamma \\
q \in \alpha \cap \gamma_{* p}}} \varepsilon_{p}(\beta, \gamma) \varepsilon_{q}(\alpha, \gamma)\left[\gamma_{* q} \alpha_{q \circ} \nu_{\circ *}\right] \otimes\left[\bar{\nu}_{* \circ} \alpha_{\circ q} \gamma_{q p} \beta_{p \bullet \nu_{\bullet *}}\right] \otimes\left[\bar{\nu}_{* \bullet} \beta_{\bullet p} \gamma_{p *}\right] \\
& +\sum_{\substack{p \in \beta \cap \gamma \\
q \in \alpha \cap \beta_{p} \bullet}} \varepsilon_{p}(\beta, \gamma) \varepsilon_{q}(\alpha, \beta)\left[\gamma_{* p} \beta_{p q} \alpha_{q \circ} \nu_{\circ *}\right] \otimes\left[\bar{\nu}_{* \circ} \alpha_{\circ q} \beta_{q \bullet} \nu_{\bullet *}\right] \otimes\left[\bar{\nu}_{* \bullet} \beta_{\bullet p} \gamma_{p *}\right] .
\end{aligned}
$$

The second term in (7.2.12) is

$$
\begin{aligned}
& -P_{132}\left\{\{\{a, b\}\}^{\eta}, c\right\}_{L}^{\eta} \\
& =-P_{132} \sum_{p \in \alpha \cap \beta} \varepsilon_{p}(\alpha, \beta)\left\{\left[\bar{\nu}_{* \bullet} \beta \bullet p \alpha_{p \circ} \nu_{\circ *}\right] \otimes\left[\bar{\nu}_{* \circ} \alpha_{\circ p} \beta_{p \bullet} \nu_{\bullet *}\right], c\right\}_{L}^{\eta} \\
& \left.=-P_{132} \sum_{p \in \alpha \cap \beta} \varepsilon_{p}(\alpha, \beta)\left\{\left[\bar{\nu}_{* \bullet} \beta \bullet p \alpha_{p \circ} \nu_{\circ *}\right], c\right\}\right\}^{\eta} \otimes\left[\bar{\nu}_{* \circ} \alpha_{\circ p} \beta_{p \bullet} \nu_{\bullet *}\right] \\
& =-P_{132} \sum_{\substack{p \in \alpha \cap \beta \\
q \in \beta \bullet p \cap \gamma}} \varepsilon_{p}(\alpha, \beta) \varepsilon_{q}(\beta, \gamma)\left[\gamma_{* q} \beta_{q p} \alpha_{p \circ} \nu_{\circ *}\right] \otimes\left[\bar{\nu}_{* \bullet} \beta \bullet q \gamma_{q *}\right] \otimes\left[\bar{\nu}_{* \circ} \alpha_{\circ p} \beta_{p \bullet \nu \bullet *}\right] \\
& -P_{132} \sum_{\substack{p \in \alpha \cap \beta \\
q \in \alpha_{p \circ} \cap \gamma}} \varepsilon_{p}(\alpha, \beta) \varepsilon_{q}(\alpha, \gamma)\left[\gamma_{* q} \alpha_{q \circ} \nu_{\circ *}\right] \otimes\left[\bar{\nu}_{* \bullet} \beta_{\bullet p} \alpha_{p q} \gamma_{q *}\right] \otimes\left[\bar{\nu}_{* \circ} \alpha_{\circ p} \beta_{p \bullet} \nu_{\bullet *}\right] \\
& =-\sum_{\substack{p \in \alpha \cap \beta \\
q \in \beta \bullet p \cap \gamma}} \varepsilon_{p}(\alpha, \beta) \varepsilon_{q}(\beta, \gamma)\left[\gamma_{* q} \beta_{q p} \alpha_{p \circ} \nu_{\circ *}\right] \otimes\left[\bar{\nu}_{* \circ} \alpha_{\circ p} \beta_{p \bullet} \nu_{\bullet *}\right] \otimes\left[\bar{\nu}_{* \bullet} \beta_{\bullet q} \gamma_{q *}\right]
\end{aligned}
$$




$$
-\sum_{\substack{p \in \alpha_{\cap} \cap \beta \\ q \in \alpha_{p \circ} \cap \gamma}} \varepsilon_{p}(\alpha, \beta) \varepsilon_{q}(\alpha, \gamma)\left[\gamma_{* q} \alpha_{q \circ} \nu_{\circ *}\right] \otimes\left[\bar{\nu}_{* \circ} \alpha_{\circ p} \beta_{p \bullet} \nu_{\bullet *}\right] \otimes\left[\bar{\nu}_{* \bullet} \beta_{\bullet p} \alpha_{p q} \gamma_{q *}\right] .
$$

The third term in (7.2.12) is

$$
\begin{aligned}
-\left\{\left[b,\{\{a, c\}\}^{\eta}\right\}_{R}^{\eta}\right. & =-\sum_{p \in \alpha \cap \gamma} \varepsilon_{p}(\alpha, \gamma)\left\{\left[b,\left[\gamma_{* p} \alpha_{p \circ} \nu_{\circ *}\right] \otimes\left[\bar{\nu}_{* \circ} \alpha_{\circ p} \gamma_{p *}\right]\right\}_{R}^{\eta}\right. \\
& =-\sum_{p \in \alpha \cap \gamma} \varepsilon_{p}(\alpha, \gamma)\left[\gamma_{* p} \alpha_{p \circ} \nu_{\circ *}\right] \otimes\left\{\left[b,\left[\bar{\nu}_{* \circ} \alpha_{\circ p} \gamma_{p *}\right]\right\}\right\}^{\eta} .
\end{aligned}
$$

To finish the computation of the third term, we denote by $\alpha^{\prime}$ a loop based at $\bullet$ and $\beta^{\prime}$ a loop based at $\circ$ obtained from $\alpha$ and $\beta$ respectively by "switching" their base points along $\partial \Sigma$ : thus, $\alpha^{\prime} \cap \beta^{\prime}$ is $\alpha \cap \beta$ with four extra points. We obtain

$$
\begin{aligned}
& -\left\{\left\{b,\left\{\{a, c\}^{\eta}\right\}_{R}^{\eta}=-\sum_{p \in \alpha^{\prime} \cap \gamma} \varepsilon_{p}\left(\alpha^{\prime}, \gamma\right)\left[\gamma_{* p} \alpha_{p \bullet}^{\prime} \nu_{\bullet *}\right] \otimes\left\{\left\{b,\left[\bar{\nu}_{* \bullet} \alpha_{\bullet p}^{\prime} \gamma_{p *}\right]\right\}\right\}^{\eta}\right.\right. \\
& =-\sum_{\substack{p \in \alpha^{\prime} \cap \gamma \\
q \in \beta^{\prime} \cap \gamma_{p *}}} \varepsilon_{p}\left(\alpha^{\prime}, \gamma\right) \varepsilon_{q}\left(\beta^{\prime}, \gamma\right)\left[\gamma_{* p} \alpha_{p \bullet}^{\prime} \nu_{\bullet *}\right] \otimes\left[\bar{\nu}_{* \bullet} \alpha_{\bullet p}^{\prime} \gamma_{p q} \beta_{q \circ}^{\prime} \nu_{\circ *}\right] \otimes\left[\bar{\nu}_{* \circ} \beta_{\circ q}^{\prime} \gamma_{q *}\right] \\
& -\sum_{\substack{p \in \alpha^{\prime} \cap \gamma \\
q \in \beta^{\prime} \cap \alpha_{\bullet}^{\prime}}} \varepsilon_{p}\left(\alpha^{\prime}, \gamma\right) \varepsilon_{q}\left(\beta^{\prime}, \alpha^{\prime}\right)\left[\gamma_{* p} \alpha_{p \bullet}^{\prime} \nu_{\bullet *}\right] \otimes\left[\bar{\nu}_{* \bullet} \alpha_{\bullet q}^{\prime} \beta_{q \circ}^{\prime} \nu_{\circ *}\right] \otimes\left[\bar{\nu}_{* \circ} \beta_{\circ q}^{\prime} \alpha_{q p}^{\prime} \gamma_{p *}\right] \\
& =-\sum_{\substack{p \in \alpha \cap \gamma \\
q \in \beta \cap \gamma_{p *}}} \varepsilon_{p}(\alpha, \gamma) \varepsilon_{q}(\beta, \gamma)\left[\gamma_{* p} \alpha_{p \circ} \nu_{\circ *}\right] \otimes\left[\bar{\nu}_{* \circ} \alpha_{\circ p} \gamma_{p q} \beta_{q \bullet} \nu_{\bullet *}\right] \otimes\left[\bar{\nu}_{* \bullet} \beta_{\bullet} \gamma_{q *}\right] \\
& -\sum_{\substack{p \in \alpha \cap \gamma \\
q \in \beta \cap \alpha_{\circ p}}} \varepsilon_{p}(\alpha, \gamma) \varepsilon_{q}(\beta, \alpha)\left[\gamma_{* p} \alpha_{p \circ} \nu_{\circ *}\right] \otimes\left[\bar{\nu}_{* \circ} \alpha_{\circ q} \beta_{q \bullet} \nu_{\bullet *}\right] \otimes\left[\bar{\nu}_{* \bullet} \beta_{\bullet q} \alpha_{q p} \gamma_{p *}\right] \\
& -\sum_{p \in \alpha \cap \gamma} \varepsilon_{p}(\alpha, \gamma)\left[\gamma_{* p} \alpha_{p \circ} \nu_{\circ *}\right] \otimes b \otimes\left[\bar{\nu}_{* \circ} \alpha_{\circ p} \gamma_{p *}\right] \\
& +\sum_{p \in \alpha \cap \gamma} \varepsilon_{p}(\alpha, \gamma)\left[\gamma_{* p} \alpha_{p \circ} \nu_{\circ *}\right] \otimes 1 \otimes b\left[\bar{\nu}_{* \circ} \alpha_{\circ p} \gamma_{p *}\right] \\
& =-\sum_{\substack{p \in \alpha \cap \gamma \\
q \in \beta \cap \gamma_{p *}}} \varepsilon_{p}(\alpha, \gamma) \varepsilon_{q}(\beta, \gamma)\left[\gamma_{* p} \alpha_{p \circ} \nu_{\circ *}\right] \otimes\left[\bar{\nu}_{* \circ} \alpha_{\circ p} \gamma_{p q} \beta_{q \bullet} \nu_{\bullet *}\right] \otimes\left[\bar{\nu}_{* \bullet} \beta_{\bullet q} \gamma_{q *}\right] \\
& -\sum_{\substack{p \in \alpha \cap \gamma \\
q \in \beta \cap \alpha_{\circ p}}} \varepsilon_{p}(\alpha, \gamma) \varepsilon_{q}(\beta, \alpha)\left[\gamma_{* p} \alpha_{p \circ} \nu_{\circ *}\right] \otimes\left[\bar{\nu}_{* \circ} \alpha_{\circ q} \beta_{q \bullet} \nu_{\bullet *}\right] \otimes\left[\bar{\nu}_{* \bullet} \beta_{\bullet q} \alpha_{q p} \gamma_{p *}\right] \\
& -P_{132}\left(\{\{a, c\}\}^{\eta} \otimes b\right)+P_{132}\left(b *\{\{a, c\}\}^{\eta} \otimes 1\right) .
\end{aligned}
$$

Summing up these expressions for the first three terms of (7.2.12), and canceling pairwise the six sums indexed by $(p, q)$, we obtain (7.2.12) and (7.2.5).

7.3. Proof of Theorem 3.1. By Lemma 6.2, the skew-symmetrized homotopy intersection pairing $\eta^{s}$ induces a double bracket $\left\{\{-,-\}^{s}\right.$ on $A=\mathbb{K} \pi$. By Lemma 7.2. this double bracket is quasi-Poisson. For each $N \geq 1$, we endow the $\left(G_{N}, \mathfrak{g}_{N}\right)$ algebra $A_{N}$ with the bracket $\{-,-\}$ derived from $\left\{\{-,-\}^{s}\right.$ via Lemma 4.1. In the 
notation of Lemma 7.1, this bracket is computed by

$$
\begin{aligned}
& \left\{a_{i j}, b_{u v}\right\}=2 \sum_{p \in \alpha \cap \beta} \varepsilon_{p}(\alpha, \beta)\left[\beta_{* p} \alpha_{p \bullet} \nu_{\bullet *}\right]_{u j}\left[\bar{\nu}_{* \bullet} \alpha \bullet p \beta_{p *}\right]_{i v} \\
& +\delta_{u j}(a b)_{i v}+\delta_{i v}(b a)_{u j}-a_{u j} b_{i v}-b_{u j} a_{i v}
\end{aligned}
$$

for all $a, b \in \pi$ and $i, j, u, v \in\{1, \ldots, N\}$. According to Lemma 4.4, this turns $A_{N}$ into a quasi-Poisson algebra and proves the first claim of Theorem 3.1

As explained in Sections 4.34 .5 , the quasi-Poisson double bracket $\{[-,-\}\}^{s}$ in $A$ induces a Lie bracket $\langle-,-\rangle$ in $\check{A}=A /[A, A]$. Recall from Section 3.3 that $\check{A}=\mathbb{K} \check{\pi}$ is the free $\mathbb{K}$-module whose basis $\check{\pi}$ can be identified with the set of free homotopy classes of loops in $\Sigma$. Formulas (4.3.1), (7.2.2) and (7.2.3) imply that for any generic loops $\alpha, \beta$ in $\Sigma$,

$$
\langle\alpha, \beta\rangle=2 \sum_{p \in \alpha \cap \beta} \varepsilon_{p}(\alpha, \beta) \alpha_{p} \beta_{p}
$$

where $\alpha_{p} \beta_{p}$ is the product of the loops $\alpha$ and $\beta$ based at $p$. The right-hand side of this formula is 2 times the Goldman Lie bracket in $\check{A}$. Therefore the second claim of Theorem 3.1 follows directly from Lemma 4.2

7.4. Remarks. 1. The bracket $\langle-,-\rangle: A \times A \rightarrow A$ associated to the double bracket $\left\{\{-,-\}^{s}\right.$ by formula (4.3.1) is twice the form $\sigma$ introduced by Kawazumi and Kuno in KK1]. An operation similar to $\{-,-\}^{\eta}: A \otimes A \rightarrow A \otimes A$ has been independently introduced by Kawazumi and Kuno KK2 for other purposes.

2. When $2=0$ in $\mathbb{K}$, the quasi-Poisson double bracket in $A=\mathbb{K} \pi$ simplifies to

$$
\left\{\{a, b\}^{s}=1 \otimes a b+b a \otimes 1+a \otimes b+b \otimes a\right.
$$

and the quasi-Poisson bracket $\{-,-\}$ on $A_{N}$ simplifies to

$$
\left\{a_{i j}, b_{u v}\right\}=\delta_{u j}(a b)_{i v}+\delta_{i v}(b a)_{u j}+a_{u j} b_{i v}+b_{u j} a_{i v} .
$$

The latter formula does not involve topology of the surface and yields a quasiPoisson bracket on $A_{N}$ for any algebra $A$ (provided $2=0$ in $\mathbb{K}$ ).

3 . Consider the case where $\Sigma$ is a compact connected oriented surface of genus $g \geq 1$ with $\partial \Sigma \cong S^{1}$. For $\mathbb{K}=\mathbb{Q}$, the F-pairing $\eta$ admits a tensorial description in terms of a symplectic expansion of $\pi$, see $\mathrm{Mas}$, $\mathrm{MT}$. In a sequel to this paper, the authors will derive a tensorial description of the double bracket $\left\{\{-,-\}^{s}\right.$ and use it to relate this bracket to the Poisson double bracket associated by Van den Bergh $[\mathrm{VdB}]$ to the quiver having one vertex and $g$ edges. This will allow us to relate the cobracket of loops in $\Sigma$ introduced by the second-named author to the cobracket of loops in quivers introduced by T. Schedler.

4. A part of the results of this paper has an analogue for oriented smooth manifolds with boundary of dimension $\geq 3$. Given such a manifold $M$ with base point $* \in \partial M$, consider the space $\Omega=\Omega(M, *)$ of loops in $M$ based at $*$. Suppose that the ground ring $\mathbb{K}$ is a field. Since $\Omega$ is an $H$-group, its homology $A=H_{*}(\Omega ; \mathbb{K})$ has a natural structure of a graded Hopf algebra. Imitating the formula (7.2.3) in the context of string topology of Chas and Sullivan [CS, we define a double bracket in $A$. This double bracket induces a graded Poisson bracket in the associated commutative graded algebras $\left\{A_{N}\right\}_{N \geq 1}$. One can view $A_{N}$ as the "coordinate algebra" of the "scheme" of graded algebra homomorphisms $A \rightarrow \operatorname{Mat}_{N}(B)$ where $B$ runs over all commutative graded algebras. If $M$ is simply connected and $\mathbb{K}$ is a field of characteristic zero, then by the Milnor-Moore theorem, $A$ is the universal 
enveloping algebra of the graded Lie algebra $\oplus_{i \geq 2} \pi_{i}(M, *) \otimes \mathbb{K}$ with Whitehead product in the role of the graded Lie bracket. Thus, $A_{N}$ is the "coordinate algebra" of the "scheme" of Lie algebra homomorphisms from $\oplus_{i \geq 2} \pi_{i}(M, *) \otimes \mathbb{K}$ to $\operatorname{Mat}_{N}(B)$ where $B$ runs over all commutative graded algebras. These results will be discussed in detail in a sequel to this paper.

5. The constructions of this paper can be generalized to the case of surfaces with several marked points on the boundary. This generalization involves representations of the fundamental groupoid of the surface based at those points. We plan to study this general case in another place.

\section{Quasi-Poisson Structures on the RePresentation MANifolds}

In this section we construct a quasi-Poisson structure on the representation manifold associated with a compact oriented surface with boundary. We begin by recalling the notion of a quasi-Poisson manifold following [AKsM]. Throughout this section we assume that $\mathbb{K}=\mathbb{R}$.

8.1. Quasi-Poisson manifolds. We first fix a few conventions. For a vector space $V$ over $\mathbb{R}$, we denote by $\Lambda V$ the exterior algebra of $V$, i.e., the quotient of the tensor algebra $\oplus_{n \geq 0} V^{\otimes n}$ by the two-sided ideal generated by the set $\{v \otimes v\}_{v \in V}$. As usual, multiplication in $\Lambda V=\oplus_{n \geq 0} \Lambda^{n} V$ is denoted by the symbol $\wedge$. We define a linear $\operatorname{map} \iota: \Lambda V \rightarrow \oplus_{n \geq 0} V^{\otimes n}$ by

$$
\iota\left(v_{1} \wedge \cdots \wedge v_{n}\right)=\sum_{\sigma} \varepsilon(\sigma) v_{\sigma(1)} \otimes \cdots \otimes v_{\sigma(n)}
$$

for any $v_{1}, \ldots, v_{n} \in V$ with $n \geq 1$. Here $\sigma$ runs over all permutations of the set $\{1, \ldots, n\}$ and $\varepsilon(\sigma)$ is the sign of $\sigma$. The map $\iota$ is an isomorphism of $\Lambda V$ onto the subspace of $\oplus_{n \geq 0} V^{\otimes n}$ consisting of all skew-symmetric tensors.

Consider a smooth manifold $M$ and let $T=T(M)$ be its tangent bundle. Applying the functor $V \mapsto \Lambda^{n} V$ fiber-wise we obtain a smooth vector bundle $\Lambda^{n} T$ over $M$ for all $n \geq 0$. Let $C^{\infty}\left(\Lambda^{n} T\right)$ be the vector space of smooth sections of $\Lambda^{n} T$. The elements of $C^{\infty}\left(\Lambda^{n} T\right)$ are called $n$-vector fields on $M$. The direct sum $C^{\infty}(\Lambda T)=\oplus_{n \geq 0} C^{\infty}\left(\Lambda^{n} T\right)$ is a graded algebra with respect to the pointwise exterior multiplication $\wedge$. Here $C^{\infty}\left(\Lambda^{0} T\right)=C^{\infty}(M)$ is the algebra of smooth $\mathbb{R}$-valued functions on $M$ and $C^{\infty}\left(\Lambda^{1} T\right)=C^{\infty}(T)$ is the vector space of smooth tangent vector fields on $M$.

The Schouten-Nijenhuis bracket on $M$ is the $\mathbb{R}$-bilinear map

$$
[-,-]: C^{\infty}(\Lambda T) \times C^{\infty}(\Lambda T) \rightarrow C^{\infty}(\Lambda T)
$$

uniquely determined by the following conditions (see, for example, $\mathrm{Mar}$ ):

(1) for any $f, g \in C^{\infty}(M)$, we have $[f, g]=0$;

(2) for any $X \in C^{\infty}(T)$ and $U \in C^{\infty}(\Lambda T)$, the bracket $[X, U]$ is the Lie derivative of $U$ along the vector field $X$;

(3) for any $U \in C^{\infty}\left(\Lambda^{k} T\right), V \in C^{\infty}\left(\Lambda^{l} T\right)$ with $k, l \geq 0$ and $W \in C^{\infty}(\Lambda T)$,

$$
\begin{gathered}
{[U, V]=-(-1)^{(k-1)(l-1)}[V, U],} \\
{[U, V \wedge W]=[U, V] \wedge W+(-1)^{(k-1) l} V \wedge[U, W] .}
\end{gathered}
$$

Set $\mathcal{A}=C^{\infty}(M)$ and let $T^{*}=T^{*}(M)$ be the cotangent bundle of $M$. Consider the algebra of differential forms on $M$

$$
C^{\infty}\left(\Lambda T^{*}\right)=\oplus_{n \geq 0} C^{\infty}\left(\Lambda^{n} T^{*}\right)
$$


with the exterior multiplication $\wedge$. There is a natural $\mathcal{A}$-bilinear pairing $\langle-,-\rangle$ from $C^{\infty}\left(\Lambda T^{*}\right) \times C^{\infty}(\Lambda T)$ to $\mathcal{A}$ which is uniquely determined by the condition that $\left\langle C^{\infty}\left(\Lambda^{n} T^{*}\right), C^{\infty}\left(\Lambda^{k} T\right)\right\rangle=0$ for $n \neq k$ and

$$
\left\langle\alpha_{1} \wedge \cdots \wedge \alpha_{k}, X_{1} \wedge \cdots \wedge X_{k}\right\rangle=\operatorname{det}\left(\left\langle\alpha_{i}, X_{j}\right\rangle\right)_{i, j=1}^{k}
$$

for all differential 1-forms $\alpha_{1}, \ldots, \alpha_{k}$ and vector fields $X_{1}, \ldots, X_{k}$ on $M$ with $k \geq 0$. We use the pairing $\langle-,-\rangle$ to associate with each $k$-vector field $U \in C^{\infty}\left(\Lambda^{k} T\right)$ a skew-symmetric multiderivation $\{-, \cdots,-\}_{U}: \mathcal{A}^{k} \rightarrow \mathcal{A}$ by

$$
\left\{f_{1}, \ldots, f_{k}\right\}_{U}=\left\langle d f_{1} \wedge \cdots \wedge d f_{k}, U\right\rangle
$$

for any $f_{1}, \ldots, f_{k} \in \mathcal{A}$.

Suppose now that $M$ is endowed with a smooth (left) action of a Lie group $G$. This induces an action of $G$ on the algebra $\mathcal{A}=C^{\infty}(M)$ by

$$
(g f)(m)=f\left(g^{-1} m\right)
$$

for $g \in G, f \in \mathcal{A}, m \in M$. The action of $G$ on $M$ also induces an action of the Lie algebra, $\mathfrak{g}$, of $G$ on $\mathcal{A}$ by

$$
(v f)(m)=\left.\frac{d}{d t}\right|_{t=0} f\left(e^{-t v} m\right)
$$

for $v \in \mathfrak{g}, f \in \mathcal{A}, m \in M$. It is easy to check that $\mathcal{A}$ is a $(G, \mathfrak{g})$-algebra in the sense of Section 2.3 .

For any $v \in \mathfrak{g}$, the derivation (8.1.2) of $\mathcal{A}$ corresponds to a smooth tangent vector field on $M$ denoted by $v_{M}$ and said to be generated by $v$. Note that $([v, w])_{M}=$ $\left[v_{M}, w_{M}\right]$ for any $v, w \in \mathfrak{g}$. The linear map $\mathfrak{g} \rightarrow C^{\infty}(T), v \mapsto v_{M}$ extends uniquely to an algebra homomorphism $\Lambda \mathfrak{g} \rightarrow C^{\infty}(\Lambda T)$. In this way, any $k$-vector $\Phi \in \Lambda^{k} \mathfrak{g}$ with $k \geq 0$ generates a $k$-vector field on $M$ denoted $\Phi_{M}$. The action (8.1.1) extends to an action of $G$ on $C^{\infty}(\Lambda T)$ by $g U=\Lambda\left(d \gamma_{g}\right) \circ U \circ \gamma_{g^{-1}}$, for all $U \in C^{\infty}(\Lambda T)$ and any $g \in G$ whose action on $M$ is denoted here by $\gamma_{g}: M \rightarrow M$. It is easy to check that the map $\Lambda \mathfrak{g} \rightarrow C^{\infty}(\Lambda T), \Phi \mapsto \Phi_{M}$ is $G$-equivariant. In particular, a multivector field on $M$ generated by a $G$-invariant vector in $\Lambda \mathfrak{g}$ is also $G$-invariant.

Suppose finally that the Lie algebra $\mathfrak{g}$ is endowed with a $G$-invariant nondegenerate symmetric bilinear form $\cdot: \mathfrak{g} \times \mathfrak{g} \rightarrow \mathbb{R}$ and consider the associated Cartan trivector $\phi \in \wedge_{\mathfrak{g}}^{3}$, defined at (2.2.2). (Recall that $\wedge_{\mathfrak{g}}^{3}$ is the vector space of $\mathfrak{g}$-invariant skew-symmetric elements of $\mathfrak{g}^{\otimes 3}$.) By a quasi-Poisson structure on $M$ we shall mean a $G$-invariant bivector field $P \in C^{\infty}\left(\Lambda^{2} T\right)$ such that the SchoutenNijenhuis bracket $[P, P] \in C^{\infty}\left(\Lambda^{3} T\right)$ is equal to the $G$-invariant trivector field $\left(\iota^{-1}(2 \phi)\right)_{M}=2\left(\iota^{-1}(\phi)\right)_{M}$ on $M$ generated by $\iota^{-1}(2 \phi)=2 \iota^{-1}(\phi) \in \Lambda^{3} \mathfrak{g}$. We call such a $P$ a quasi-Poisson bivector field on $M$. Note that, given dual bases $\left(e_{i}\right)_{i}$ and $\left(e_{i}^{\sharp}\right)_{i}$ of $\mathfrak{g}$ (so that $e_{i} \cdot e_{j}^{\sharp}=\delta_{i j}$ for all $i, j$ ), we have

$$
\iota^{-1}(\phi)=\iota^{-1}\left(\sum_{i, j, k}\left(e_{i} \cdot\left[e_{j}, e_{k}\right]\right) e_{i}^{\sharp} \otimes e_{j}^{\sharp} \otimes e_{k}^{\sharp}\right)=\frac{1}{6} \sum_{i, j, k}\left(e_{i} \cdot\left[e_{j}, e_{k}\right]\right) e_{i}^{\sharp} \wedge e_{j}^{\sharp} \wedge e_{k}^{\sharp} .
$$

The following lemma AKsM shows that a quasi-Poisson structure on $M$ induces on $C^{\infty}(M)$ a structure of a quasi-Poisson algebra associated with $\phi$, as defined in Section 2.3 .

Lemma 8.1. Let $M$ be a smooth manifold endowed with a smooth action of a Lie group $G$, whose Lie algebra $\mathfrak{g}$ is equipped with a $G$-invariant non-degenerate symmetric bilinear form. Let $\phi \in \wedge_{\mathfrak{g}}^{3}$ be the associated Cartan trivector and $\mathcal{A}=$ 
$C^{\infty}(M)$. Then, the pairing $\{-,-\}_{P}: \mathcal{A} \times \mathcal{A} \rightarrow \mathcal{A}$ determined by a quasi-Poisson bivector field $P$ on $M$ as above is a quasi-Poisson bracket in $\mathcal{A}$ associated with $\phi$.

Proof. Recall that any $k$-vector field $U \in C^{\infty}\left(\Lambda^{k} T\right)$ determines an $\mathcal{A}$-linear map

$$
i_{U}: C^{\infty}\left(\Lambda T^{*}\right) \rightarrow C^{\infty}\left(\Lambda T^{*}\right)
$$

of degree $-k$ called the right interior product by $U$, see, for instance, $\mathrm{Mar}$. The map $i_{U}$ is uniquely determined by the condition that $\left\langle i_{U} \alpha, X\right\rangle=\langle\alpha, U \wedge X\rangle$ for all $\alpha \in C^{\infty}\left(\Lambda^{n} T^{*}\right)$ with $n \geq k$ and all $X \in C^{\infty}\left(\Lambda^{n-k} T\right)$. By [Mar, Proposition 4.1],

$$
i_{[U, V]}=-\left[\left[i_{V}, d\right], i_{U}\right]
$$

for any $U \in C^{\infty}\left(\Lambda^{k} T\right)$ and $V \in C^{\infty}\left(\Lambda^{l} T\right)$ with $k, l \geq 0$. On the right-hand side of (8.1.3), the bracket $[\varphi, \psi]$ of two graded endomorphisms $\varphi$ and $\psi$ of $C^{\infty}\left(\Lambda T^{*}\right)$ stands for the graded commutator $\varphi \psi-(-1)^{\operatorname{deg}(\varphi) \operatorname{deg}(\psi)} \psi \varphi$. By (8.1.3), we have for any $f, g, h \in \mathcal{A}$,

$$
\begin{aligned}
\{f, g, h\}_{[P, P]} & =\langle d f \wedge d g \wedge d h,[P, P]\rangle \\
& =i_{[P, P]}(d f \wedge d g \wedge d h) \\
& =-\left[i_{P} d-d i_{P}, i_{P}\right](d f \wedge d g \wedge d h) \\
& =-\left(i_{P} d i_{P}-d i_{P} i_{P}-i_{P} i_{P} d+i_{P} d i_{P}\right)(d f \wedge d g \wedge d h) \\
& =-2 i_{P} d i_{P}(d f \wedge d g \wedge d h) .
\end{aligned}
$$

Here we use that $d(d f \wedge d g \wedge d h)=0$ and that $i_{P} i_{P}$ decreases the degree by 4 and therefore annihilates $d f \wedge d g \wedge d h$. Expanding the determinant of a $(3 \times 3)$-matrix with respect to the last row, we obtain that for any vector field $X$ on $M$,

$\langle d f \wedge d g \wedge d h, P \wedge X\rangle=\langle d f \wedge d g, P\rangle\langle d h, X\rangle-\langle d f \wedge d h, P\rangle\langle d g, X\rangle+\langle d g \wedge d h, P\rangle\langle d f, X\rangle$.

Therefore

$$
i_{P}(d f \wedge d g \wedge d h)=\{f, g\}_{P} d h+\{h, f\}_{P} d g+\{g, h\}_{P} d f .
$$

We deduce that

$$
\begin{aligned}
\{f, g, h\}_{[P, P]} & =-2 i_{P}\left(d\{f, g\}_{P} \wedge d h+d\{h, f\}_{P} \wedge d g+d\{g, h\}_{P} \wedge d f\right) \\
& =2\left(\left\{h,\{f, g\}_{P}\right\}_{P}+\left\{g,\{h, f\}_{P}\right\}_{P}+\left\{f,\{g, h\}_{P}\right\}_{P}\right)
\end{aligned}
$$

The assumption $[P, P]=2\left(\iota^{-1}(\phi)\right)_{M}$ implies that $\{-,-\}_{P}$ is a quasi-Poisson bracket in $\mathcal{A}$ associated with $\phi$.

8.2. A quasi-Poisson structure on $\mathcal{H}$. Let $\Sigma$ be a compact connected oriented surface with non-empty boundary and base point $* \in \partial \Sigma$. Then the group $\pi=$ $\pi_{1}(\Sigma, *)$ is free of a finite rank $n \geq 0$. We fix an integer $N \geq 1$ and consider the smooth manifold

$$
\mathcal{H}=\operatorname{Hom}\left(\pi, G_{N}\right) \cong\left(G_{N}\right)^{n} \quad \text { where } \quad G_{N}=\mathrm{GL}_{N}(\mathbb{R}) .
$$

The group $G_{N}$ acts on $\mathcal{H}$ by conjugations in the obvious way. The aim of this subsection is to construct a natural quasi-Poisson structure on $\mathcal{H}$.

Set $A=\mathbb{R} \pi$ and recall the algebra $A_{N}$ from Section 3, By Section 3.3, we have an evaluation map ev $=\mathrm{ev}_{\mathbb{R}}$ from $A_{N}$ to the algebra $\operatorname{Map}(\mathcal{H}, \mathbb{R})$ of $\mathbb{R}$-valued functions on $\mathcal{H}$. To describe ev in concrete terms, pick a basis $\left\{x_{u}\right\}_{u=1}^{n}$ of $\pi$ and use it to identify $\mathcal{H}=\left(G_{N}\right)^{n}$. Consider the presentation of $A_{N}$ by generators and relations discussed at the end of Section 3.3. The map ev carries the generator $x_{i j}^{u}$ (respectively, $\left.y^{u}\right)$ of $A_{N}$ to the function $\mathcal{H}=\left(G_{N}\right)^{n} \rightarrow \mathbb{R}$ sending a sequence of $n$ invertible 
matrices to the $(i, j)$-th entry of the $u$-th matrix (respectively, to the inverse of the determinant of the $u$-th matrix). We can view $\mathcal{H}$ as the affine algebraic set defined by the equations $\tilde{y}^{u} \operatorname{det}\left(\tilde{x}_{i j}^{u}\right)_{i, j}=1$ in the affine space $\left(\operatorname{Mat}_{N}(\mathbb{R}) \times \mathbb{R}\right)^{n}=\mathbb{R}^{\left(N^{2}+1\right) n}$ with coordinates $\left(\left(\tilde{x}_{i j}^{u}\right)_{i, j}, \tilde{y}^{u}\right)_{u}$. The coordinate algebra $\mathbb{R}[\mathcal{H}]$ of $\mathcal{H}$ is the quotient of $\mathbb{R}\left[\left(\left(\tilde{x}_{i j}^{u}\right)_{i, j}, \tilde{y}^{u}\right)_{u}\right]$ by the ideal generated by the polynomials $\tilde{y}^{u} \operatorname{det}\left(\tilde{x}_{i j}^{u}\right)_{i, j}-1$ for $u \in\{1, \ldots, n\}$. Restricting polynomial functions $\mathbb{R}^{\left(N^{2}+1\right) n} \rightarrow \mathbb{R}$ to $\mathcal{H} \subset \mathbb{R}^{\left(N^{2}+1\right) n}$, we identify $\mathbb{R}[\mathcal{H}]$ with a subalgebra of $C^{\infty}(\mathcal{H}) \subset \operatorname{Map}(\mathcal{H}, \mathbb{R})$. Then the description of ev above shows that ev is an isomorphism from $A_{N}$ onto $\mathbb{R}[\mathcal{H}]$. Transporting along ev the quasi-Poisson bracket in $A_{N}$ provided by Theorem 3.1 we obtain a skew-symmetric bracket $\{-,-\}$ in $\mathbb{R}[\mathcal{H}]$ which is a derivation in each variable.

Theorem 8.2. There is a unique quasi-Poisson structure on $\mathcal{H}$ such that the associated bracket in $C^{\infty}(\mathcal{H})$ extends the bracket $\{-,-\}$ in $\mathbb{R}[\mathcal{H}]$. This quasi-Poisson structure is invariant under the action of $\operatorname{Homeo}(\Sigma, *)$ on $\mathcal{H}$.

Proof. We claim that there is a unique bivector field $P \in C^{\infty}\left(\Lambda^{2} T \mathcal{H}\right)$ such that $\{f, g\}=\{f, g\}_{P}=\langle d f \wedge d g, P\rangle$ for all $f, g \in \mathbb{R}[\mathcal{H}]$. Indeed, pick a point $m \in \mathcal{H}$ and let $T_{m} \mathcal{H}$ be the tangent space of the smooth manifold $\mathcal{H}$ at $m$. Consider the maximal ideal $I_{m}$ of $\mathbb{R}[\mathcal{H}]$ consisting of all $f \in \mathbb{R}[\mathcal{H}]$ such that $f(m)=0$. Recall that at any smooth point of an affine algebraic set, the Zariski tangent space may be identified with the tangent space of differential topology. Specifically, there is a nondegenerate bilinear form $\left(I_{m} / I_{m}^{2}\right) \times T_{m} \mathcal{H} \rightarrow \mathbb{R}$ defined by $(f, v) \mapsto d_{m} f(v)$ where $f \in I_{m}$ and $v \in T_{m} \mathcal{H}$. On the other hand, the map $(f, g) \mapsto\{f, g\}(m)$, where $f, g \in$ $I_{m}$, induces a skew-symmetric bilinear form $\left(I_{m} / I_{m}^{2}\right) \times\left(I_{m} / I_{m}^{2}\right) \rightarrow \mathbb{R}$. Therefore there is a unique bivector $P_{m} \in \Lambda^{2} T_{m} \mathcal{H}$ such that $\{f, g\}(m)=\left\langle d_{m} f \wedge d_{m} g, P_{m}\right\rangle$ for all $f, g \in I_{m}$. In fact, the latter formula holds for all $f, g \in \mathbb{R}[\mathcal{H}]$ because $\{1,-\}=\{-, 1\}=0$. The map $m \mapsto P_{m}$ defines a unique section $P$ of the bundle $\Lambda^{2} T \mathcal{H}$ over $\mathcal{H}$ such that $\{f, g\}=\langle d f \wedge d g, P\rangle$ for all $f, g \in \mathbb{R}[\mathcal{H}]$. It remains to justify that $P \in C^{\infty}\left(\Lambda^{2} T \mathcal{H}\right)$, i.e., that $P$ is a smooth section. For this, we consider the functions $\tilde{x}_{i j}^{u}=\operatorname{ev}\left(x_{i j}^{u}\right) \in \mathbb{R}[\mathcal{H}]$ as above. These functions form a local system of coordinates in a neighborhood of any point of $\mathcal{H}$, and we can expand

$$
P=\frac{1}{2} \sum_{u, v, i, j, k, l}\left\langle d \tilde{x}_{i j}^{u} \wedge d \tilde{x}_{k l}^{v}, P\right\rangle \frac{\partial}{\partial \tilde{x}_{i j}^{u}} \wedge \frac{\partial}{\partial \tilde{x}_{k l}^{v}}=\frac{1}{2} \sum_{u, v, i, j, k, l}\left\{\tilde{x}_{i j}^{u}, \tilde{x}_{k l}^{v}\right\} \frac{\partial}{\partial \tilde{x}_{i j}^{u}} \wedge \frac{\partial}{\partial \tilde{x}_{k l}^{v}} .
$$

Since the functions $\left\{\tilde{x}_{i j}^{u}, \tilde{x}_{k l}^{v}\right\} \in \mathbb{R}[\mathcal{H}]$ are smooth, $P$ is a smooth bivector field on $\mathcal{H}$ such that the bracket $\{-,-\}_{P}$ in $C^{\infty}(\mathcal{H})$ extends the bracket $\{-,-\}$ in $\mathbb{R}[\mathcal{H}]$.

We verify now that $P$ is $G_{N}$-invariant. Observe that the action (8.1.1) of $G_{N}$ on $C^{\infty}(\mathcal{H})$ preserves the subalgebra $\mathbb{R}[\mathcal{H}]$. By Section 3.3. the evaluation map ev from the $\left(G_{N}, \mathfrak{g}_{N}\right)$-algebra $A_{N}$ to $C^{\infty}(\mathcal{H})$ is $G_{N}$-equivariant. This and the $G_{N}$-invariance of the quasi-Poisson bracket in $A_{N}$ imply the $G_{N}$-invariance of the bracket $\{-,-\}$ in $\mathbb{R}[\mathcal{H}]$. The latter implies the $G_{N}$-invariance of $P$.

To see that $P$ defines a quasi-Poisson structure on $\mathcal{H}$, we need only to check the modified Jacobi identity. Observe first that the action (8.1.2) of $\mathfrak{g}_{N}$ on $C^{\infty}(\mathcal{H})$ preserves $\mathbb{R}[\mathcal{H}]$ and the evaluation map ev : $A_{N} \rightarrow C^{\infty}(\mathcal{H})$ is $\mathfrak{g}_{N}$-equivariant. It suffices to check the $\mathfrak{g}_{N}$-equivariance of ev on each generator of $A_{N}$ of the form $x_{i j}^{u}$ with $u \in\{1, \ldots, n\}$ and $i, j \in\{1, \ldots, N\}$. For any $w \in \mathfrak{g}_{N}$ and any point $m=\left(m_{v}\right)_{v=1}^{n} \in \mathcal{H}=\left(G_{N}\right)^{n}$, we have

$$
\left(w \operatorname{ev}\left(x_{i j}^{u}\right)\right)(m)=\left(w \tilde{x}_{i j}^{u}\right)(m)
$$




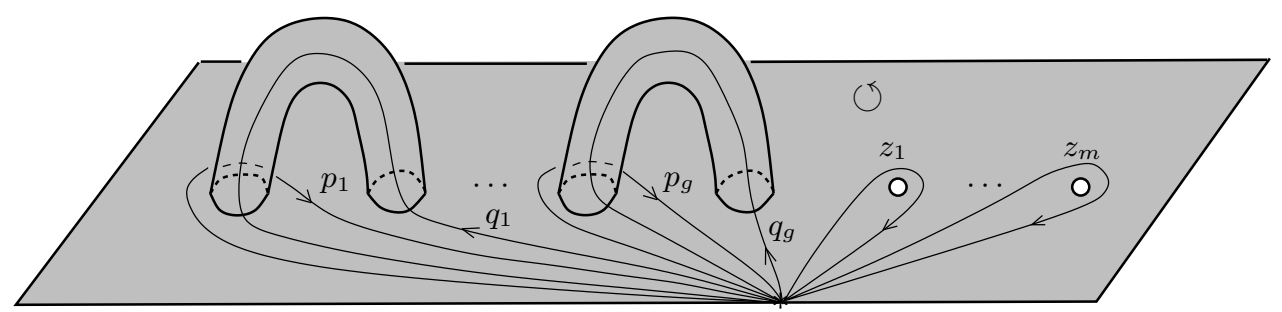

Figure 1. A surface $\Sigma$ and a basis of $\pi$.

$$
\begin{aligned}
& \left.\stackrel{\text { 8.1.2) }}{=} \frac{d}{d t}\right|_{t=0} \tilde{x}_{i j}^{u}\left(e^{-t w} m\right) \\
& =\left.\frac{d}{d t}\right|_{t=0} \tilde{x}_{i j}^{u}\left(e^{-t w} m_{1} e^{t w}, \ldots, e^{-t w} m_{n} e^{t w}\right) \\
& =\left.\frac{d}{d t}\right|_{t=0}\left(e^{-t w} m_{u} e^{t w}\right)_{i, j} \\
& =\left(m_{u} w-w m_{u}\right)_{i, j} \\
& =\left(m_{u}\right)_{i, s} w_{s, j}-w_{i, s}\left(m_{u}\right)_{s, j} \\
& =\left(\tilde{x}_{i s}^{u} w_{s, j}-w_{i, s} \tilde{x}_{s j}^{u}\right)(m) \stackrel{\text { (3.2.3. }}{=} \operatorname{ev}\left(w x_{i j}^{u}\right)(m) .
\end{aligned}
$$

Since our bracket in $A_{N}$ is a quasi-Poisson bracket associated with $\phi_{N}$, so is the bracket $\{-,-\}$ in $\mathbb{R}[\mathcal{H}]$. Thus, for any $f, g, h \in \mathbb{R}[\mathcal{H}]$,

$$
\begin{aligned}
\langle d f \wedge d g \wedge d h,[P, P]\rangle & =\{f, g, h\}_{[P, P]} \\
\stackrel{\sqrt[8.1 .4]{=}}{=} & 2\left(\left\{f,\{g, h\}_{P}\right\}_{P}+\left\{g,\{h, f\}_{P}\right\}_{P}+\left\{h,\{f, g\}_{P}\right\}_{P}\right) \\
= & 2(\{f,\{g, h\}\}+\{g,\{h, f\}\}+\{h,\{f, g\}\}) \\
= & 2 \phi_{N}(f, g, h) \\
= & 2\left\langle d f \wedge d g \wedge d h,\left(\iota^{-1}\left(\phi_{N}\right)\right)_{\mathcal{H}}\right\rangle
\end{aligned}
$$

where $\left(\iota^{-1}\left(\phi_{N}\right)\right)_{\mathcal{H}}$ is the trivector field on $\mathcal{H}$ induced by $\iota^{-1}\left(\phi_{N}\right) \in \Lambda^{3} \mathfrak{g}_{N}$. Since the differentials of regular functions fill in the cotangent space, $[P, P]=2\left(\iota^{-1}\left(\phi_{N}\right)\right)_{\mathcal{H}}$.

The invariance of $P$ under the action of $\operatorname{Homeo}(\Sigma, *)$ follows from the corresponding property of the quasi-Poisson bracket in $A_{N}$ and the fact that the evaluation map preserves this action.

8.3. Computations. We give explicit formulas for the quasi-Poisson brackets associated with a compact connected oriented surface $\Sigma$ of genus $g \geq 0$ with $m+1 \geq 1$ boundary components. Fix a basis $\left(p_{1}, q_{1}, \ldots, p_{g}, q_{g}, z_{1}, \ldots, z_{m}\right)$ of $\pi=\pi_{1}(\Sigma, *)$ as shown on Figure 1,

We first compute the double bracket $\left\{\{-,-\}^{s}\right.$ in $\mathbb{K} \pi$. For any $u, v \in\{1, \ldots, m\}$ such that $u<v$, we have $\left\{\left\{z_{u}, z_{v}\right\}\right\}^{\eta}=0$ so that

$$
\left\{\left\{z_{u}, z_{v}\right\}^{s}=1 \otimes z_{u} z_{v}+z_{v} z_{u} \otimes 1-z_{u} \otimes z_{v}-z_{v} \otimes z_{u}\right.
$$

and, for any $u \in\{1, \ldots, m\}$, we have $\left\{\left\{z_{u}, z_{u}\right\}^{\eta}=z_{u} \otimes z_{u}-1 \otimes z_{u}^{2}\right.$ so that

$$
\left\{\left\{z_{u}, z_{u}\right\}\right\}^{s}=z_{u}^{2} \otimes 1-1 \otimes z_{u}^{2} .
$$


For any $u, v \in\{1, \ldots, g\}$ with $u<v$ and any $a, b \in\{p, q\}$, we have $\left\{\left\{a_{u}, b_{v}\right\}\right\}^{\eta}=0$ so that

$$
\left\{\left\{a_{u}, b_{v}\right\}^{s}=1 \otimes a_{u} b_{v}+b_{v} a_{u} \otimes 1-a_{u} \otimes b_{v}-b_{v} \otimes a_{u} .\right.
$$

For any $u \in\{1, \ldots, g\}$, we have

$\left.\left\{\left\{p_{u}, p_{u}\right\}\right\}^{\eta}=p_{u} \otimes p_{u}-1 \otimes p_{u}^{2}, \quad\left\{\left\{q_{u}, q_{u}\right\}\right\}^{\eta}=q_{u} \otimes q_{u}-q_{u}^{2} \otimes 1, \quad\left\{p_{u}, q_{u}\right\}\right\}^{\eta}=q_{u} \otimes p_{u}$

so that

$$
\left\{\left\{p_{u}, p_{u}\right\}\right\}^{s}=p_{u}^{2} \otimes 1-1 \otimes p_{u}^{2}, \quad\left\{\left\{q_{u}, q_{u}\right\}\right\}^{s}=1 \otimes q_{u}^{2}-q_{u}^{2} \otimes 1,
$$

and

$$
\left\{\left\{p_{u}, q_{u}\right\}^{s}=1 \otimes p_{u} q_{u}+q_{u} p_{u} \otimes 1-p_{u} \otimes q_{u}+q_{u} \otimes p_{u} .\right.
$$

For any $u \in\{1, \ldots, g\}$ and $v \in\{1, \ldots, m\}$, we have $\left\{\left\{p_{u}, z_{v}\right\}\right\}^{\eta}=0$ so that

$$
\left\{\left\{p_{u}, z_{v}\right\}\right\}^{s}=1 \otimes p_{u} z_{v}+z_{v} p_{u} \otimes 1-p_{u} \otimes z_{v}-z_{v} \otimes p_{u} .
$$

For any $u \in\{1, \ldots, g\}$ and $v \in\{1, \ldots, m\}$, we have $\left\{\left\{q_{u}, z_{v}\right\}\right\}^{\eta}=0$ so that

$$
\left\{\left\{q_{u}, z_{v}\right\}^{s}=1 \otimes q_{u} z_{v}+z_{v} q_{u} \otimes 1-q_{u} \otimes z_{v}-z_{v} \otimes q_{u} .\right.
$$

We now fix an $N \geq 1$. By Section 3.3, given a basis $\left(x_{1}, x_{2}, \ldots, x_{2 g+m}\right)$ of $\pi$, the quasi-Poisson bracket $\{-,-\}$ in $(\mathbb{K} \pi)_{N}$ produced by Theorem 3.1 is determined by its values on the generators $x_{i j}^{u}=\left(x_{u}\right)_{i j}$ with $u \in\{1, \ldots, 2 g+m\}$ and $i, j \in$ $\{1, \ldots, N\}$. Applying this fact to the chosen basis of $\pi$, we deduce from the computations above the following formulas which hold for arbitrary $i, j, k, l \in\{1, \ldots, N\}$. For any $u, v \in\{1, \ldots, m\}$ with $u<v$,

$$
\left\{z_{i j}^{u}, z_{k l}^{v}\right\}=\delta_{k j} z_{i r}^{u} z_{r l}^{v}+z_{k s}^{v} z_{s j}^{u} \delta_{i l}-z_{k j}^{u} z_{i l}^{v}-z_{k j}^{v} z_{i l}^{u} .
$$

For any $u \in\{1, \ldots, m\}$,

$$
\left\{z_{i j}^{u}, z_{k l}^{u}\right\}=z_{k r}^{u} z_{r j}^{u} \delta_{i l}-\delta_{k j} z_{i s}^{u} z_{s l}^{u}
$$

For any $u, v \in\{1, \ldots, g\}$ with $u<v$ and any $a, b \in\{p, q\}$,

$$
\left\{a_{i j}^{u}, b_{k l}^{v}\right\}=\delta_{k j} a_{i r}^{u} b_{r l}^{v}+b_{k s}^{v} a_{s j}^{u} \delta_{i l}-a_{k j}^{u} b_{i l}^{v}-b_{k j}^{v} a_{i l}^{u} .
$$

For any $u \in\{1, \ldots, g\}$,

$$
\begin{aligned}
& \left\{p_{i j}^{u}, p_{k l}^{u}\right\}=p_{k r}^{u} p_{r j}^{u} \delta_{i l}-\delta_{k j} p_{i s}^{u} p_{s l}^{u} \\
& \left\{q_{i j}^{u}, q_{k l}^{u}\right\}=\delta_{k j} q_{i s}^{u} q_{s l}^{u}-q_{k r}^{u} q_{r j}^{u} \delta_{i l} \\
& \left\{p_{i j}^{u}, q_{k l}^{u}\right\}=\delta_{k j} p_{i r}^{u} q_{r l}^{u}+q_{k s}^{u} p_{s j}^{u} \delta_{i l}-p_{k j}^{u} q_{i l}^{u}+q_{k j}^{u} p_{i l}^{u}
\end{aligned}
$$

For any $u \in\{1, \ldots, g\}$ and $v \in\{1, \ldots, m\}$,

$$
\begin{aligned}
& \left\{p_{i j}^{u}, z_{k l}^{v}\right\}=\delta_{k j} p_{i r}^{u} z_{r l}^{v}+z_{k s}^{v} p_{s j}^{u} \delta_{i l}-p_{k j}^{u} z_{i l}^{v}-z_{k j}^{v} p_{i l}^{u} \\
& \left\{q_{i j}^{u}, z_{k l}^{v}\right\}=\delta_{k j} q_{i r}^{u} z_{r l}^{v}+z_{k s}^{v} q_{s j}^{u} \delta_{i l}-q_{k j}^{u} z_{i l}^{v}-z_{k j}^{v} q_{i l}^{u}
\end{aligned}
$$

For $\mathbb{K}=\mathbb{R}$, we can use a basis $\left(x_{1}, x_{2}, \ldots, x_{2 g+m}\right)$ of $\pi$ to identify the space $\mathcal{H}=\operatorname{Hom}\left(\pi, \mathrm{GL}_{N}(\mathbb{R})\right)$ with the open subset $\left(\mathrm{GL}_{N}(\mathbb{R})\right)^{n}$ of $\left(\operatorname{Mat}_{N}(\mathbb{R})\right)^{n}=\mathbb{R}^{n N^{2}}$. The functions $\left(\tilde{x}_{i j}^{u}\right)_{u, i, j}$ form a system of smooth coordinates on $\mathcal{H}$ where $u \in$ $\{1, \ldots, 2 g+m\}, i, j \in\{1, \ldots, N\}$, and $\tilde{x}_{i j}^{u}: \mathcal{H} \rightarrow \mathbb{R}$ carries a tuple of $2 g+n$ matrices to the $(i, j)$-th entry of the $u$-th matrix. In the coordinates derived in 
this way from the chosen basis of $\pi$, the quasi-Poisson structure on $\mathcal{H}$ provided by Theorem 8.2 is determined by the bivector field

$$
\begin{aligned}
& P=\sum_{\substack{u<v \\
i, j, k, l}}\left\{\tilde{z}_{i j}^{u}, \tilde{z}_{k l}^{v}\right\} \frac{\partial}{\partial \tilde{z}_{i j}^{u}} \wedge \frac{\partial}{\partial \tilde{z}_{k l}^{v}}+\frac{1}{2} \sum_{u, i, j, k, l}\left\{\tilde{z}_{i j}^{u}, \tilde{z}_{k l}^{u}\right\} \frac{\partial}{\partial \tilde{z}_{i j}^{u}} \wedge \frac{\partial}{\partial \tilde{z}_{k l}^{u}} \\
& +\sum_{\substack{u<v \\
i, j, k, l}}\left(\left\{\tilde{p}_{i j}^{u}, \tilde{p}_{k l}^{v}\right\} \frac{\partial}{\partial \tilde{p}_{i j}^{u}} \wedge \frac{\partial}{\partial \tilde{p}_{k l}^{v}}+\left\{\tilde{q}_{i j}^{u}, \tilde{q}_{k l}^{v}\right\} \frac{\partial}{\partial \tilde{q}_{i j}^{u}} \wedge \frac{\partial}{\partial \tilde{q}_{k l}^{v}}\right) \\
& +\sum_{\substack{u<v \\
i, j, k, l}}\left(\left\{\tilde{p}_{i j}^{u}, \tilde{q}_{k l}^{v}\right\} \frac{\partial}{\partial \tilde{p}_{i j}^{u}} \wedge \frac{\partial}{\partial \tilde{q}_{k l}^{v}}+\left\{\tilde{q}_{i j}^{u}, \tilde{p}_{k l}^{v}\right\} \frac{\partial}{\partial \tilde{q}_{i j}^{u}} \wedge \frac{\partial}{\partial \tilde{p}_{k l}^{v}}\right) \\
& +\frac{1}{2} \sum_{u, i, j, k, l}\left(\left\{\tilde{p}_{i j}^{u}, \tilde{p}_{k l}^{u}\right\} \frac{\partial}{\partial \tilde{p}_{i j}^{u}} \wedge \frac{\partial}{\partial \tilde{p}_{k l}^{u}}+\left\{\tilde{q}_{i j}^{u}, \tilde{q}_{k l}^{u}\right\} \frac{\partial}{\partial \tilde{q}_{i j}^{u}} \wedge \frac{\partial}{\partial \tilde{q}_{k l}^{u}}\right) \\
& +\sum_{u, i, j, k, l}\left\{\tilde{p}_{i j}^{u}, \tilde{q}_{k l}^{u}\right\} \frac{\partial}{\partial \tilde{p}_{i j}^{u}} \wedge \frac{\partial}{\partial \tilde{q}_{k l}^{u}} \\
& +\sum_{\substack{u, v \\
i, j, k, l}}\left(\left\{\tilde{p}_{i j}^{u}, \tilde{z}_{k l}^{v}\right\} \frac{\partial}{\partial \tilde{p}_{i j}^{u}} \wedge \frac{\partial}{\partial \tilde{z}_{k l}^{v}}+\left\{\tilde{q}_{i j}^{u}, \tilde{z}_{k l}^{v}\right\} \frac{\partial}{\partial \tilde{q}_{i j}^{u}} \wedge \frac{\partial}{\partial \tilde{z}_{k l}^{v}}\right) .
\end{aligned}
$$

Here the brackets $\{-,-\}$ are computed from (8.3.1) - (8.3.8) by replacing all $p$ 's, $q$ 's and $z$ 's with $\tilde{p}$ 's, $\tilde{q}$ 's and $\tilde{z}$ 's respectively.

8.4. Remarks. 1. The restriction of the quasi-Poisson bracket $\{-,-\}$ in $C^{\infty}(\mathcal{H})$ to the subalgebra of $G_{N}$-invariant elements $C^{\infty}(\mathcal{H})^{G_{N}}$ is a Poisson bracket, and the map ev $\circ$ tr $: \mathbb{R} \check{\pi} \rightarrow \mathbb{R}[\mathcal{H}]^{G_{N}} \subset C^{\infty}(\mathcal{H})^{G_{N}}$ carries the Goldman bracket in $\mathbb{R} \check{\pi}$ into $(1 / 2)\{-,-\}$. The restriction of $\{-,-\}$ to $\mathbb{R}[\mathcal{H}]^{G_{N}}$ can be uniquely recovered from the Goldman bracket because the set $\operatorname{ev}(\operatorname{tr}(\check{\pi}))$ generates the algebra $\mathbb{R}[\mathcal{H}]^{G_{N}}$.

2. Our definition of a quasi-Poisson structure on a manifold differs from that in AKsM by a factor of 2: a quasi-Poisson bivector field in our sense is 2 times a quasi-Poisson bivector field in the sense of [AKsM].

3. Under the assumptions of Section 8.3 the isomorphism class of $(\mathcal{H}, P)$ (in the category of quasi-Poisson manifolds) does not depend on the choice of the base point $* \in \partial \Sigma$. This follows from the naturality of our bracket under surface homeomorphisms and the fact that for any points $*, *^{\prime} \in \partial \Sigma$, there is an orientationpreserving self-homeomorphism of $\Sigma$ carrying $*$ to $*^{\prime}$.

\section{Moment maps AND SURFACES Without Boundary}

We discuss moment maps and, as an application, associate certain Poisson algebras with surfaces without boundary.

9.1. Moment maps. Motivated by the notion of a multiplicative moment map for a quasi-Poisson manifold [AKsM], Van den Bergh [VdB] defined a similar notion for a quasi-Poisson double bracket $\{\{-,-\}$ in any algebra $A$. In our notation, his definition may be reformulated as follows: a moment map for $\{\{-,-\}$ is an invertible element $\mu \in A$ such that for all $a \in A$,

$$
\{\{\mu, a\}=a \otimes \mu+a \mu \otimes 1-\mu \otimes a-1 \otimes \mu a .
$$


Let $N \geq 1$ be an integer. Formula (9.1.1) allows us to compute the bracket $\left\{\left(\mu^{m}\right)_{i j}, a_{u v}\right\} \in A_{N}$ for any $a \in A, m \in \mathbb{Z}$, and $i, j, u, v \in\{1, \ldots, N\}$. For $m>0$,

$$
\begin{aligned}
\left\{\left\{\mu^{m}, a\right\}\right\} & =\left\{\left\{\mu^{m-1} \mu, a\right\}\right\} \\
& =\mu^{m-1} *\left\{\{\mu, a\}+\left\{\left\{\mu^{m-1}, a\right\}\right\} * \mu\right. \\
& =a \otimes \mu^{m}+a \mu \otimes \mu^{m-1}-\mu \otimes \mu^{m-1} a-1 \otimes \mu^{m} a+\left\{\left\{\mu^{m-1}, a\right\}\right\} * \mu \\
& =\sigma_{0, m}(\mu, a)+\sigma_{1, m-1}(\mu, a)+\left\{\left\{\mu^{m-1}, a\right\}\right\} * \mu
\end{aligned}
$$

where $\sigma_{k, m-k}(\mu, a)=a \mu^{k} \otimes \mu^{m-k}-\mu^{k} \otimes \mu^{m-k} a$ for any $k \in\{0, \ldots, m\}$. Since $\sigma_{k, m-1-k}(\mu, a) * \mu=\sigma_{k+1, m-(k+1)}(\mu, a)$, we deduce recursively that

$$
\left\{\left\{\mu^{m}, a\right\}=\sigma_{0, m}(\mu, a)+\sigma_{m, 0}(\mu, a)+2 \sum_{k=1}^{m-1} \sigma_{k, m-k}(\mu, a) .\right.
$$

Therefore

$$
\begin{aligned}
\left\{\left(\mu^{m}\right)_{i j}, a_{u v}\right\}= & a_{u j}\left(\mu^{m}\right)_{i v}-\delta_{u j}\left(\mu^{m} a\right)_{i v}+\left(a \mu^{m}\right)_{u j} \delta_{i v}-\left(\mu^{m}\right)_{u j} a_{i v} \\
& +2 \sum_{k=1}^{m-1}\left(\left(a \mu^{k}\right)_{u j}\left(\mu^{m-k}\right)_{i v}-\left(\mu^{k}\right)_{u j}\left(\mu^{m-k} a\right)_{i v}\right) .
\end{aligned}
$$

Similar formulas hold for the $(-m)$-th power of $\mu$ (where $m>0$ ). Set $\bar{\mu}=\mu^{-1} \in A$. Using the equalities $\left\{\left\{\bar{\mu}^{m}, a\right\}\right\}=-\bar{\mu}^{m} *\left\{\left\{\mu^{m}, a\right\}\right\} * \bar{\mu}^{m}$ and

$$
\bar{\mu}^{m} * \sigma_{k, m-k}(\mu, a) * \bar{\mu}^{m}=\sigma_{m-k, k}(\bar{\mu}, a),
$$

we deduce from (9.1.2) that

$$
\left\{\left\{\bar{\mu}^{m}, a\right\}=-\sigma_{0, m}(\bar{\mu}, a)-\sigma_{m, 0}(\bar{\mu}, a)-2 \sum_{k=1}^{m-1} \sigma_{k, m-k}(\bar{\mu}, a) .\right.
$$

Hence

$$
\begin{aligned}
\left\{\left(\bar{\mu}^{m}\right)_{i j}, a_{u v}\right\}= & -a_{u j}\left(\bar{\mu}^{m}\right)_{i v}+\delta_{u j}\left(\bar{\mu}^{m} a\right)_{i v}-\left(a \bar{\mu}^{m}\right)_{u j} \delta_{i v}+\left(\bar{\mu}^{m}\right)_{u j} a_{i v} \\
& +2 \sum_{k=1}^{m-1}\left(-\left(a \bar{\mu}^{k}\right)_{u j}\left(\bar{\mu}^{m-k}\right)_{i v}+\left(\bar{\mu}^{k}\right)_{u j}\left(\bar{\mu}^{m-k} a\right)_{i v}\right) .
\end{aligned}
$$

These computations imply, in particular, that the subalgebra of $A_{N}$ generated by the $\left(\mu^{m}\right)_{i j}$ 's (with $m \in \mathbb{Z}$ and $i, j \in\{1, \ldots, N\}$ ) is closed under the bracket $\{-,-\}$.

The next lemma gives another property of moment maps used below.

Lemma 9.1. Let $\{\{-,-\}$ be a quasi-Poisson double bracket in an algebra $A$ and let $\langle-,-\rangle: \check{A} \times \check{A} \rightarrow \check{A}$ be the induced Lie bracket. Let $\mu \in A$ be a moment map, let $A^{\prime}=A / A(\mu-1) A$ be the quotient of $A$ by the two-sided ideal generated by $\mu-1$ and let $\check{A}^{\prime}=A^{\prime} /\left[A^{\prime}, A^{\prime}\right]$. Let $p: A \rightarrow A^{\prime}$ be the projection and $\check{p}: \check{A} \rightarrow \check{A}^{\prime}$ be the induced map. Then, for any $N \geq 1$, there is a unique Poisson bracket $\{-,-\}^{\prime}$ in the subalgebra $\left(A^{\prime}\right)_{N}^{t}$ of $A_{N}^{\prime}$ generated by $\operatorname{tr}\left(\check{A}^{\prime}\right) \subset A_{N}^{\prime}$ such that the following diagram commutes:

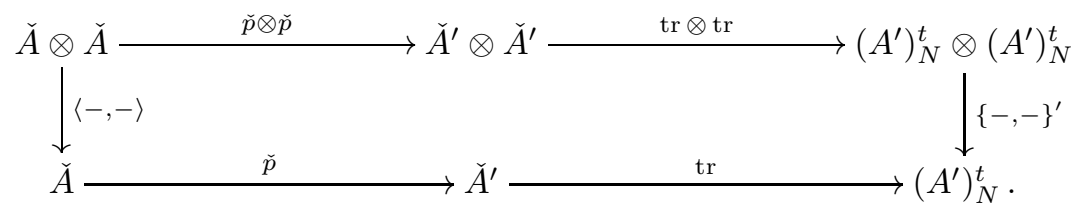


Proof. Set $T=\left(A^{\prime}\right)_{N}^{t}$. The uniqueness of a Poisson bracket in $T$ satisfying the conditions of the lemma is obvious because by the Leibniz rule, the values of such a bracket on generators of $T$ determine the bracket. The existence can be deduced from $\left[\mathrm{VdB}\right.$, Proposition 5.1.5] and [Cb, Theorem 4.5] using $H_{0}$-Poisson structures, but we rather give a direct proof. Consider the algebra map $p_{N}: A_{N} \rightarrow A_{N}^{\prime}$ induced by $p$ and the algebras $B^{\prime}=\left(A_{N}^{\prime}\right)^{\mathfrak{g}_{N}} \subset A_{N}^{\prime}$ and $B=p_{N}^{-1}\left(B^{\prime}\right) \subset A_{N}$. The following two claims are verified below:

(i) $\{B, B\} \subset B$ where $\{-,-\}$ is the quasi-Poisson bracket in $A_{N}$ induced by the quasi-Poisson double bracket $\{\{-,-\}$ in $A$, and

(ii) there is a unique bilinear pairing $\{-,-\}^{\prime}: B^{\prime} \times B^{\prime} \rightarrow B^{\prime}$ such that the following diagram commutes:

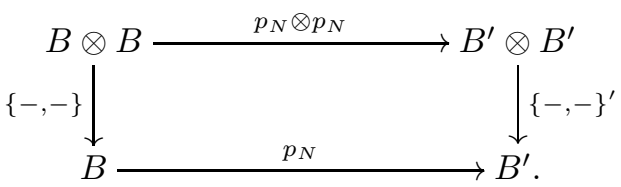

Then the properties of $\{-,-\}$ and the triviality of the action of $\mathfrak{g}_{N}$ on $B^{\prime}$ imply that $\{-,-\}^{\prime}$ is a Poisson bracket in $B^{\prime}$. Clearly, $T \subset B^{\prime}$. For any $a, b \in \check{A}$, we have

$$
\begin{aligned}
\{\operatorname{tr} \check{p}(a), \operatorname{tr} \check{p}(b)\}^{\prime} & =\left\{p_{N} \operatorname{tr}(a), p_{N} \operatorname{tr}(b)\right\}^{\prime}=p_{N}\{\operatorname{tr}(a), \operatorname{tr}(b)\} \\
& =p_{N} \operatorname{tr}(\langle a, b\rangle)=\operatorname{tr} \check{p}(\langle a, b\rangle)
\end{aligned}
$$

where we use the commutativity of the diagram 9.1.6) and Lemma 4.2. Thus we have $\{T, T\}^{\prime} \subset T$, and the restriction of $\{-,-\}^{\prime}$ to $T \subset B^{\prime}$ is a Poisson bracket in $T$ satisfying the conditions of the lemma.

To prove the claims (i), (ii), we observe that for all $y \in A_{N}$ and $i, j \in\{1, \ldots, N\}$,

$$
p_{N}\left\{(\mu-1)_{i j}, y\right\}=2 p_{N}\left(f_{j i} y\right)
$$

where $f_{i j} \in \mathfrak{g}_{N}$ is the elementary matrix whose $(i, j)$-th entry is 1 and the other entries are 0. Since both sides of (9.1.7) are derivations in $y$, it is enough to check it for each generator $y=a_{u v}$ where $a \in A$ and $u, v \in\{1, \ldots, N\}$. We have

$$
\begin{aligned}
p_{N}\left\{(\mu-1)_{i j}, a_{u v}\right\} & =p_{N}\left(\{\mu-1, a\}_{u j}^{(1)}\left\{\{\mu-1, a\}_{i v}^{(2)}\right)\right. \\
& =p_{N}\left(\{\mu, a\}_{u j}^{(1)}\left\{\{\mu, a\}_{i v}^{(2)}\right)\right. \\
& =p_{N}\left(a_{u j} \mu_{i v}+(a \mu)_{u j} \delta_{i v}-\mu_{u j} a_{i v}-\delta_{u j}(\mu a)_{i v}\right) \\
& =p_{N}\left(a_{u j}\right) \delta_{i v}+p_{N}\left(a_{u j}\right) \delta_{i v}-\delta_{u j} p_{N}\left(a_{i v}\right)-\delta_{u j} p_{N}\left(a_{i v}\right) \\
& =2 p_{N}\left(f_{j i} a_{u v}\right) .
\end{aligned}
$$

We now prove (i). Observe that

$$
B=p_{N}^{-1}\left(\left(A_{N}^{\prime}\right)^{\mathfrak{g}_{N}}\right)=\left\{x \in A_{N}: w x \in \operatorname{Ker} p_{N} \text { for all } w \in \mathfrak{g}_{N}\right\} .
$$

Let us pick any $x, y \in B$ and show that $\{x, y\} \in B$. We need to show that $w\{x, y\} \in \operatorname{Ker} p_{N}$ for all $w \in \mathfrak{g}_{N}$. By the definition of $p_{N}$, the ideal Ker $p_{N} \subset A_{N}$ is generated by the elements $(\mu-1)_{i j}=\mu_{i j}-\delta_{i j}$ with $i, j \in\{1, \ldots, N\}$. Since $x, y \in B$, we have $w x=\sum_{a} r_{a}(\mu-1)_{i_{a} j_{a}}$ and $w y=\sum_{b} s_{b}(\mu-1)_{k_{b} l_{b}}$ where $a, b$ run over finite sets of indices, $r_{a}, s_{b} \in A_{N}$, and $i_{a}, j_{a}, k_{b}, l_{b} \in\{1, \ldots, N\}$. Then

$$
w\{x, y\}=\{w x, y\}+\{x, w y\}=\sum_{a}\left\{r_{a}, y\right\}(\mu-1)_{i_{a} j_{a}}+\sum_{a} r_{a}\left\{(\mu-1)_{i_{a} j_{a}}, y\right\}
$$




$$
+\sum_{b}\left\{x, s_{b}\right\}(\mu-1)_{k_{b} l_{b}}+\sum_{b} s_{b}\left\{x,(\mu-1)_{k_{b} l_{b}}\right\} .
$$

The following is deduced from (9.1.7) and the fact that $x, y \in B$ :

$$
p_{N}(w\{x, y\})=2 \sum_{a} p_{N}\left(r_{a}\right) p_{N}\left(f_{j_{a} i_{a}} y\right)+2 \sum_{b} p_{N}\left(s_{b}\right) p_{N}\left(f_{l_{b} k_{b}} x\right)=0 .
$$

We now verify (ii). Since the map $\left.p_{N}\right|_{B}: B \rightarrow B^{\prime}$ is surjective and the bracket $\{-,-\}$ is skew-symmetric, it is enough to verify that $p_{N}\{x, y\}=0$ for any $x \in$ $\operatorname{Ker} p_{N}$ and $y \in B$. It suffices to check the case where $x=r(\mu-1)_{i j}$ with $r \in A_{N}$ and $i, j \in\{1, \ldots, N\}$. Then, applying (9.1.7) again, we obtain that

$$
p_{N}\{x, y\}=p_{N}\left(\{r, y\}(\mu-1)_{i j}+r\left\{(\mu-1)_{i j}, y\right\}\right)=2 p_{N}(r) p_{N}\left(f_{j i} y\right)=0 .
$$

9.2. Peripheral loops. Let $\Sigma$ be an oriented surface with base point $* \in \partial \Sigma$, such that the component of $\partial \Sigma$ containing $*$ is a circle. (This is always the case if $\Sigma$ is compact.) Let $\nu \in \pi=\pi_{1}(\Sigma, *)$ be represented by this circle component viewed as a loop based at $*$ with orientation induced from that of $\Sigma$. Then $\bar{\nu}=\nu^{-1} \in \pi$ is a moment map for the quasi-Poisson double bracket $\left\{\{-,-\}^{s}\right.$ in $\mathbb{K} \pi$ introduced in Section 7 . Indeed, by formula (7.2.3), we have $\{\bar{\nu}, a\}^{\eta}=a \otimes \bar{\nu}-1 \otimes \bar{\nu} a$ for all $a \in \pi$ so that

$$
\left\{[\bar{\nu}, a\}^{s}=a \otimes \bar{\nu}+a \bar{\nu} \otimes 1-\bar{\nu} \otimes a-1 \otimes \bar{\nu} a .\right.
$$

Thus, formulas (9.1.2) generated by the $\left(\nu^{k}\right)_{i j}$ 's (with $k \in \mathbb{Z}$ and $i, j \in\{1, \ldots, N\}$ ) is closed under the bracket can also be deduced from the naturality of $\{\{-,-\}\}^{s}$ under inclusions of surfaces: if $\Sigma$ is not a 2-disk, then $\nu \in \pi$ is an element of infinite order, and the subalgebra in question is the $N$-th quasi-Poisson algebra associated with the annulus $S^{1} \times[0,1]$.

9.3. Arbitrary oriented surfaces. Let $\Sigma$ be an oriented surface possibly without boundary. Pick a point $* \in \Sigma$ and set $\pi=\pi_{1}(\Sigma, *)$ and $A=\mathbb{K} \pi$. If $* \notin \partial \Sigma$, then we do not have a pairing in $A$ similar to the pairing of Section 7.1 and consecutively have neither a corresponding double bracket in $A$ nor a quasi-Poisson bracket in $A_{N}$. However, for any choice of $*$, the subalgebra $A_{N}^{t}$ of $A_{N}$ generated by $\operatorname{tr}(\check{A}) \subset A_{N}$ has a natural Poisson bracket which we now define. Recall first the Goldman Lie bracket $\langle-,-\rangle_{\mathrm{G}}$ in $\check{A}=A /[A, A]=\mathbb{K} \check{\pi}$. By definition, for any $a, b \in \check{\pi}$,

$$
\langle a, b\rangle_{\mathrm{G}}=\sum_{p \in \alpha \cap \beta} \varepsilon_{p}(\alpha, \beta) \alpha_{p} \beta_{p}
$$

where $\alpha, \beta$ are generic loops in $\Sigma$ representing $a, b$ and $\alpha_{p} \beta_{p} \in \check{\pi}$ is represented by the product of the loops $\alpha, \beta$ based at $p$.

Theorem 9.2. For any $N \geq 1$, the algebra $A_{N}^{t}$ has a unique Poisson bracket $\{-,-\}$ such that $\{\operatorname{tr} a, \operatorname{tr} b\}=2 \operatorname{tr}\left(\langle a, b\rangle_{\mathrm{G}}\right)$ for all $a, b \in \check{A}$.

Proof. The uniqueness of $\{-,-\}$ is obvious and we need only to prove the existence. When $* \in \partial \Sigma$, our quasi-Poisson bracket in $A_{N}$ restricts to a Poisson bracket in $A_{N}^{\mathfrak{g}_{N}}$ which further restricts to a Poisson bracket in $A_{N}^{t}$ satisfying the conditions of the theorem. Suppose that $* \in \Sigma \backslash \partial \Sigma$. Let $\Sigma_{\circ}$ be the oriented surface obtained from $\Sigma$ by removing the interior of a closed embedded 2-disk $D \subset \Sigma \backslash \partial \Sigma$ such that $* \in \partial D$. Set $A_{\circ}=\mathbb{K} \pi_{\circ}$ where $\pi_{\circ}=\pi_{1}\left(\Sigma_{\circ}, *\right)$. The algebra homomorphism 
$A_{\circ} \rightarrow A$ induced by the inclusion $\Sigma_{\circ} \subset \Sigma$ is surjective, and its kernel is the twosided ideal generated by $\bar{\nu}-1$ where $\bar{\nu} \in \pi_{\circ}$ is the homotopy class of $\partial D$ with the orientation induced from that of $D \subset \Sigma$. By Lemma 9.1, the quasi-Poisson double bracket $\left\{\{-,-\}^{s}\right.$ in $A_{\circ}$ induces a Poisson bracket in $A_{N}^{t}$. Since the Lie bracket in $\check{A}_{\circ}$ induced by $\{\{-,-\}\}^{s}$ is twice the Goldman bracket of $\Sigma_{\circ}$, this Poisson bracket in $A_{N}^{t}$ satisfies the conditions of the theorem.

If $\Sigma$ is connected, then a different choice of a base point in $\Sigma$ results in a Poisson algebra canonically isomorphic to $\left(A_{N}^{t},\{-,-\}\right)$. Indeed, a path $\gamma$ in $\Sigma$ leading from $*$ to $*^{\prime} \in \Sigma$ determines an algebra isomorphism $\gamma_{\#}: A \rightarrow A^{\prime}=\mathbb{K} \pi_{1}\left(\Sigma, *^{\prime}\right)$. The induced isomorphism $A_{N} \rightarrow A_{N}^{\prime}$ carries $A_{N}^{t}$ onto $\left(A^{\prime}\right)_{N}^{t}$. Since the isomorphism $\check{\gamma}_{\#}: \check{A} \rightarrow \check{A}^{\prime}$ preserves the Goldman bracket and does not depend on the choice of $\gamma$, the resulting isomorphism $A_{N}^{t} \rightarrow\left(A^{\prime}\right)_{N}^{t}$ preserves the Poisson bracket and does not depend on the choice of $\gamma$.

When $\mathbb{K}$ is a field of characteristic zero and $\Sigma$ is compact, $A_{N}^{t}=A_{N}^{G_{N}}$ (see the end of Section 3.2) and Theorem 9.2 yields a Poisson bracket in $A_{N}^{G_{N}}$. When $\mathbb{K}=\mathbb{R}$ or $\mathbb{K}=\mathbb{C}$ this is twice the bracket studied in Go2.

\section{Generalization to Fuchsian Groups}

In this last section, we generalize Theorems 3.1 and 9.2 to so-called weighted surfaces and briefly discuss connections with Fuchsian groups.

10.1. Weighted surfaces. Let $\Sigma$ be an oriented surface with $\partial \Sigma \neq \emptyset$ endowed with base point $*$ (possibly not lying on $\partial \Sigma$ ). Note that each circle component $X$ of $\partial \Sigma$ determines a conjugacy class $[X]$ of elements of $\pi=\pi_{1}(\Sigma, *)$. A typical representative of this class has the form $\alpha \beta \alpha^{-1}$ where $\alpha$ is a path from $*$ to a point of $X$ and $\beta$ is a loop going once along $X$ in the direction determined by the orientation of $\Sigma$.

A weight on $\Sigma$ is a map from the set of circle components of $\partial \Sigma \backslash\{*\}$ to $\mathbb{Z}$. Given a weight $n$ on $\Sigma$, consider the normal subgroup $\langle n\rangle \subset \pi$ generated by all elements of the form $x^{n(X)}$ where $X$ runs over the circle components of $\partial \Sigma \backslash\{*\}$ and $x$ runs over $[X] \subset \pi$. The quotient group $\pi^{\prime}=\pi /\langle n\rangle$ is called the group of the weighted surface $(\Sigma, n)$. Set $A=\mathbb{K} \pi, A^{\prime}=\mathbb{K} \pi^{\prime}$, and let $p: A \rightarrow A^{\prime}$ be the algebra homomorphism induced by the projection $\pi \rightarrow \pi^{\prime}$. Recall that

$$
\check{A}=A /[A, A]=\mathbb{K} \check{\pi} \quad \text { and } \quad \check{A}^{\prime}=A^{\prime} /\left[A^{\prime}, A^{\prime}\right]=\mathbb{K} \check{\pi}^{\prime},
$$

and denote by $\check{p}: \check{A} \rightarrow \check{A}^{\prime}$ the linear map induced by $p$.

We show that brackets associated with $A$ induce corresponding brackets for $A^{\prime}$. Our first observation is that the Goldman Lie bracket $\langle-,-\rangle_{\mathrm{G}}$ in $\check{A}$ induces a unique Lie bracket $\langle-,-\rangle_{\mathrm{G}}^{\prime}$ in $\check{A}^{\prime}$ such that the following diagram commutes:

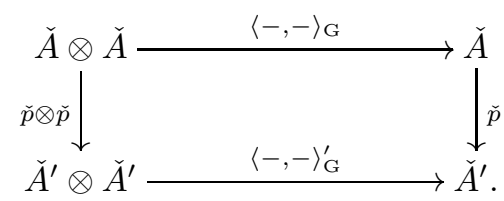

This follows from the fact that, for any circle component $X$ of $\partial \Sigma \backslash\{*\}$, all integral powers of elements of $[X]$ are central for the Goldman bracket of $\Sigma$. 
Theorem 10.1. Suppose that $* \in \partial \Sigma$. Then the following claims hold.

(i) Let $N \geq 1$ and $p_{N}: A_{N} \rightarrow A_{N}^{\prime}$ be the algebra homomorphism induced by $p$. Let $\{-,-\}$ be the quasi-Poisson bracket in $A_{N}$ produced by Theorem 3.1. There is a unique map $\{-,-\}^{\prime}: A_{N}^{\prime} \times A_{N}^{\prime} \rightarrow A_{N}^{\prime}$ such that the following diagram commutes:

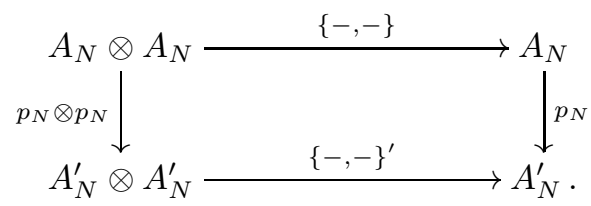

The map $\{-,-\}^{\prime}$ is a quasi-Poisson bracket in the $\left(G_{N}, \mathfrak{g}_{N}\right)$-algebra $A_{N}^{\prime}$.

(ii) For all $N \geq 1$, the trace map $\operatorname{tr}: \check{A}^{\prime} \rightarrow\left(A_{N}^{\prime}\right)^{\mathfrak{g}_{N}}$ is a homomorphism of Lie algebras where $\check{A}^{\prime}$ is endowed with the Lie bracket $2\langle-,-\rangle_{\mathrm{G}}^{\prime}$ and $\left(A_{N}^{\prime}\right)^{\mathfrak{g}_{N}}$ is endowed with the restriction of the bracket $\{-,-\}^{\prime}$.

Proof. The uniqueness of $\{-,-\}^{\prime}$ follows from the surjectivity of $p_{N}$. Since the bracket $\{-,-\}$ is quasi-Poisson, the commutativity of (10.1.2) implies that the bracket $\{-,-\}^{\prime}$ is also quasi-Poisson. We need only to prove the existence of $\{-,-\}^{\prime}$. We begin by proving that there is a unique pairing $\eta^{\prime}: A^{\prime} \times A^{\prime} \rightarrow A^{\prime}$ such that the following diagram commutes:

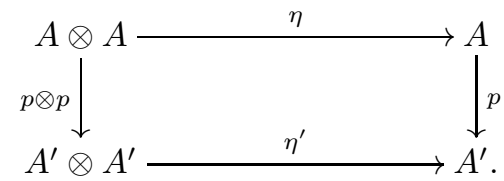

The uniqueness of $\eta^{\prime}$ follows from the surjectivity of $p$, and we need only to prove the existence of $\eta^{\prime}$. Consider the two-sided ideal Ker $p$ of $A$. We shall prove that $p \eta(\operatorname{Ker} p, A)=0$. As a left ideal, Ker $p$ is generated by the expressions of type $x^{n(X)}-1$ where $X$ runs over the circle components of $\partial \Sigma \backslash\{*\}$ and $x$ runs over $[X] \subset \pi$. Since $\eta$ is a left Fox derivative in the first variable, it suffices to prove that $p \eta\left(x^{n(X)}, A\right)=0$ for all $X$ and $x \in[X]$. Let $x=\alpha \beta \alpha^{-1}$ where $\alpha$ is a path from $*$ to a point of $X$ and $\beta$ is a loop in $X$. Clearly, $x^{n(X)}=\alpha \beta^{n(X)} \alpha^{-1}$. Any loop $\gamma$ in $\Sigma$ based at $*$ can be deformed to avoid $\beta$ and to meet $\alpha$ transversely in a finite set of simple points. A direct application of (7.1.1) shows that the contribution of each of these points to $\eta\left(x^{n(X)}, \gamma\right)=\eta\left(\alpha \beta^{n(X)} \alpha^{-1}, \gamma\right)$ has the form $\pm\left(a-x^{n(X)} a\right)$ for some $a \in \pi$. Therefore $p \eta\left(x^{n(X)}, \gamma\right)=0$. A similar argument shows that $p \eta(A, \operatorname{Ker} p)=0$. This implies the existence of $\eta^{\prime}$.

The properties of $\eta$ recalled in Section 7 imply that $\eta^{\prime}$ is an F-pairing and $\eta^{\prime}+\overline{\eta^{\prime}}=$ $-\rho_{1}$. The double bracket $\left\{\{-,-\}^{\prime}\right.$ in $A^{\prime}$ defined by the skew-symmetric F-pairing $\eta^{\prime}-\overline{\eta^{\prime}}=2 \eta^{\prime}+\rho_{1}$ in $A^{\prime}$ makes the following diagram commute:

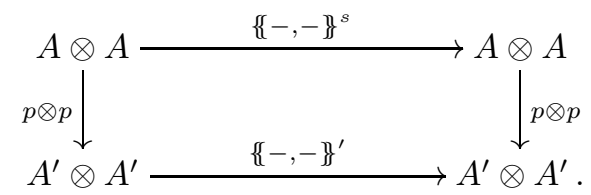

Lemma 7.2 implies that $\left\{\{-,-\}^{\prime}\right.$ is quasi-Poisson. It induces a quasi-Poisson bracket $\{-,-\}^{\prime}$ in $A_{N}^{\prime}$ by Lemma 4.1. The commutativity of the diagram (10.1.4) implies the commutativity of the diagram (10.1.2). 
The second claim of the theorem is proved following the lines of Section 7.3 .

The next theorem is an analogue of Theorem 9.2 and is proved similarly. This theorem covers all choices of $*$ in $\Sigma$.

Theorem 10.2. For any $N \geq 1$, the subalgebra $\left(A^{\prime}\right)_{N}^{t} \subset A_{N}^{\prime}$ generated by $\operatorname{tr}\left(\check{A}^{\prime}\right)$ has a unique Poisson bracket $\{-,-\}^{\prime}$ such that $\{\operatorname{tr} a, \operatorname{tr} b\}^{\prime}=2 \operatorname{tr}\left(\langle a, b\rangle_{\mathrm{G}}^{\prime}\right)$ for all $a, b \in \check{A}^{\prime}$.

All the brackets discussed in this section are natural with respect to the action of orientation-preserving weight-preserving self-homeomorphisms of the pair $(\Sigma, *)$.

10.2. Examples. 1. Let $\Sigma$ be a compact connected oriented surface of genus $g \geq 0$ with $m+1 \geq 2$ boundary components. Picking a base point on one of these components and assigning integers $n_{1}, \ldots, n_{m}$ to the other components we obtain a weight on $\Sigma$. The group, $\pi^{\prime}$, of the resulting weighted surface is presented by $2 g+m$ generators $p_{1}, q_{1}, \ldots, p_{g}, q_{g}, z_{1}, \ldots, z_{m}$ and the relations $z_{1}^{n_{1}}=\cdots=z_{m}^{n_{m}}=1$. This is a Fuchsian group with at least one parabolic or hyperbolic generator. Theorem 10.1 yields a natural quasi-Poisson bracket in the algebra $\left(\mathbb{K} \pi^{\prime}\right)_{N}$ for all $N \geq 1$. For the generators chosen as on Figure 1 the product $\left[p_{1}, q_{1}\right] \cdots\left[p_{g}, q_{g}\right] z_{1} \cdots z_{m} \in \pi^{\prime}$ is a moment map for the double bracket in $\mathbb{K} \pi^{\prime}$ defined in the proof of Theorem 10.1 .

2. Let $\Sigma$ be a compact connected oriented surface of genus $g \geq 0$ with $m \geq 1$ boundary components. Picking $* \in \Sigma \backslash \partial \Sigma$ and assigning integers $n_{1}, \ldots, n_{m}$ to the boundary components we obtain a weight on $\Sigma$. The group, $\pi^{\prime}$, of the resulting weighted surface is a Fuchsian group with generators $p_{1}, q_{1}, \ldots, p_{g}, q_{g}, z_{1}, \ldots, z_{m}$ subject to the relations $z_{1}^{n_{1}}=\cdots=z_{m}^{n_{m}}=1$ and $\left[p_{1}, q_{1}\right] \cdots\left[p_{g}, q_{g}\right] z_{1} \cdots z_{m}=1$. Theorem 10.2 yields a natural Poisson bracket in the algebra $\left(\mathbb{K} \pi^{\prime}\right)_{N}^{t}$ for all $N \geq 1$. When $\mathbb{K}$ is a field of characteristic zero, this gives a natural Poisson bracket in the algebra $\left(\mathbb{K} \pi^{\prime}\right)_{N}^{\mathrm{GL}_{N}(\mathbb{K})}$.

\section{Appendix A. The aCtions of $G_{N}$ And $\mathfrak{g}_{N}$ ON $A_{N}$ RE-EXAmined}

Given an algebra $A$ and an integer $N \geq 1$, denote by $\mathcal{X}_{N}^{A}$ the affine scheme (over $\mathbb{K})$ whose set of $B$-points $\mathcal{X}_{N}^{A}(B)$ is $\operatorname{Hom}_{\mathcal{A l g}}\left(A, \operatorname{Mat}_{N}(B)\right)$ for any commutative algebra $B$. The coordinate algebra of $\mathcal{X}_{N}^{A}$ is the commutative algebra $A_{N}$ introduced in Section 3.1. In this appendix, we analyze from the viewpoint of group schemes the actions of $G_{N}=\mathrm{GL}_{N}(\mathbb{K})$ and $\mathfrak{g}_{N}=\mathfrak{g l}_{N}(\mathbb{K})$ on $A_{N}$ defined in Section 3.2. For the language of group schemes, we refer the reader to [Ja].

For any commutative algebra $B$, the group $\operatorname{GL}_{N}(B)$ acts on $\operatorname{Mat}_{N}(B)$ by the conjugation $M \mapsto g M g^{-1}$ where $M \in \operatorname{Mat}_{N}(B)$ and $g \in \operatorname{GL}_{N}(B)$. This induces an action of $\mathrm{GL}_{N}(B)$ on $\operatorname{Hom}_{\mathcal{A l g}}\left(A, \operatorname{Mat}_{N}(B)\right)$ which is natural in $B$. These actions constitute an action of the group scheme $\mathrm{GL}_{N}$ on the affine scheme $\mathcal{X}_{N}^{A}$.

Lemma A.1. The action (3.2.2) of $G_{N}=\mathrm{GL}_{N}(\mathbb{K})$ on $A_{N}$ is induced by the action of $\mathrm{GL}_{N}$ on $\mathcal{X}_{N}^{A}$. The action (3.2.3) of $\mathfrak{g}_{N} \simeq \operatorname{Lie}\left(\mathrm{GL}_{N}\right)$ on $A_{N}$ is the infinitesimal version of the action of $\mathrm{GL}_{N}$ on $\mathcal{X}_{N}^{A}$.

Proof. For any commutative algebra $K$, the action of the group scheme $\mathrm{GL}_{N}$ on $\mathcal{X}_{N}^{A}$ induces an action of $\mathrm{GL}_{N}(K)$ on $A_{N} \otimes K$ by $K$-algebra automorphisms as follows. Consider the affine scheme over $K$ obtained from $\mathcal{X}_{N}^{A}$ by changing the 
coefficients from $\mathbb{K}$ to $K$. We can view $A_{N} \otimes K$ as the coordinate algebra of this scheme through the canonical isomorphism

$$
\operatorname{Hom}_{K-\mathcal{C A} l g}\left(A_{N} \otimes K, B\right) \simeq \operatorname{Hom}_{\mathcal{C A} l g}\left(A_{N}, B\right)
$$

for any commutative $K$-algebra $B$. Then

$$
r(g x)=\left(g^{-1} r\right)(x)
$$

for all $r \in \operatorname{Hom}_{K-\mathcal{C A l} g}\left(A_{N} \otimes K, B\right), g \in \mathrm{GL}_{N}(K)$ and $x \in A_{N} \otimes K$. Here, the action of $\mathrm{GL}_{N}(K)$ on $\operatorname{Hom}_{K-\mathcal{C A l g}}\left(A_{N} \otimes K, B\right)$ is induced by the action of $\mathrm{GL}_{N}(B)$ on $\mathcal{X}_{N}^{A}(B)$ using (A.0.1), (3.1.1), and the canonical map $\mathrm{GL}_{N}(K) \rightarrow \mathrm{GL}_{N}(B)$. This yields an action of $\mathrm{GL}_{N}(K)$ on $A_{N} \otimes K$. Applying A.0.2 to $B=A_{N} \otimes K$, $r=\operatorname{id}_{A_{N} \otimes K}$ and $x=a_{i j} \otimes 1$ (with $a \in A$ and $i, j \in\{1, \ldots, N\}$ ), we obtain

$$
g\left(a_{i j} \otimes 1\right)=\left(g^{-1} \operatorname{id}_{A_{N} \otimes K}\right)\left(a_{i j} \otimes 1\right)=a_{k l} \otimes\left(g^{-1}\right)_{i, k} g_{l, j}
$$

for any $g \in \mathrm{GL}_{N}(K)$. For $K=\mathbb{K}$, we recover the action (3.2.2) of $G_{N}$ on $A_{N}$.

The action of $\mathrm{GL}_{N}(K)$ on $A_{N} \otimes K$ is natural in $K$ and determines thus an action of the group scheme $\mathrm{GL}_{N}$ on $A_{N}$. Recall that an action of a group scheme is equivalent to a comodule over its coordinate Hopf algebra, see [Ja, §I.2.8]. Hence we obtain a comodule structure in $A_{N}$, which is given by a linear map $\Delta_{N}: A_{N} \rightarrow A_{N} \otimes \mathbb{K}\left[\mathrm{GL}_{N}\right]$ where $\mathbb{K}\left[\mathrm{GL}_{N}\right]$ is the coordinate algebra of $\mathrm{GL}_{N}$. Since $\mathrm{GL}_{N}$ acts on $A_{N}$ by algebra automorphisms, $\Delta_{N}$ is an algebra homomorphism. We now compute $\Delta_{N}$ on the generator $a_{i j} \in A_{N}$ for any $a \in A$ and $i, j \in\{1, \ldots, N\}$. We have $\Delta_{N}\left(a_{i j}\right)=\operatorname{id}_{\mathbb{K}\left[\mathrm{GL}_{N}\right]}\left(a_{i j} \otimes 1\right)$ where the action of $\operatorname{id}_{\mathbb{K}\left[\mathrm{GL}_{N}\right]} \in \operatorname{Hom}_{\mathcal{C A l g}}\left(\mathbb{K}\left[\mathrm{GL}_{N}\right], \mathbb{K}\left[\mathrm{GL}_{N}\right]\right)=\mathrm{GL}_{N}\left(\mathbb{K}\left[\mathrm{GL}_{N}\right]\right)$ is given by (A.0.3) with $K=\mathbb{K}\left[\mathrm{GL}_{N}\right]$. The matrix id $\mathrm{K}_{\mathbb{K}\left[\mathrm{GL}_{N}\right]}$ is equal to $\left(x_{i j}\right)_{i, j}$ where $x_{i j} \in \mathbb{K}\left[\mathrm{GL}_{N}\right]$ maps any $M \in \mathrm{GL}_{N}(B)$ to the $(i, j)$-th entry of $M$ for any commutative algebra $B$, and $\left(\mathrm{id}_{\mathbb{K}\left[\mathrm{GL}_{N}\right]}\right)^{-1}$ is the matrix $\left(\bar{x}_{i j}\right)_{i, j}$ where $\bar{x}_{i j} \in \mathbb{K}\left[\mathrm{GL}_{N}\right]$ maps any $M \in \mathrm{GL}_{N}(B)$ to the $(i, j)$-th entry of $M^{-1}$. Therefore

$$
\Delta_{N}\left(a_{i j}\right)=a_{k l} \otimes \bar{x}_{i k} x_{l j} .
$$

Recall that the Lie algebra of a group scheme is its tangent space at the unit point, and any representation of a group scheme carries the structure of a module over the corresponding Lie algebra, see [Ja, §I.7]. The latter structure is the "infinitesimal version" of the representation of the group scheme. We have

$$
\operatorname{Lie}\left(\mathrm{GL}_{N}\right)=\left\{\mu \in \operatorname{Hom}\left(\mathbb{K}\left[\mathrm{GL}_{N}\right], \mathbb{K}\right): \mu(1)=0, \mu\left(I_{1}^{2}\right)=0\right\} \simeq \operatorname{Hom}\left(I_{1} / I_{1}^{2}, \mathbb{K}\right)
$$

where $I_{1} \subset \mathbb{K}\left[\mathrm{GL}_{N}\right]$ is the ideal consisting of all $x \in \mathbb{K}\left[\mathrm{GL}_{N}\right]$ such that $x(1)=$ 0 . Viewing $\mathbb{K}$ as a $\mathbb{K}\left[\mathrm{GL}_{N}\right]$-module via the map $\mathbb{K}\left[\mathrm{GL}_{N}\right] \rightarrow \mathbb{K}, x \mapsto x(1)$, we can identify elements of $\operatorname{Lie}\left(\mathrm{GL}_{N}\right)$ with derivations $\mathbb{K}\left[\mathrm{GL}_{N}\right] \rightarrow \mathbb{K}$. The action of $\mathrm{Lie}\left(\mathrm{GL}_{N}\right)$ on $A_{N}$ induced by the action of $\mathrm{GL}_{N}$ on $A_{N}$ carries any $\mu \in \operatorname{Lie}\left(\mathrm{GL}_{N}\right)$ to the composite map

$$
A_{N} \stackrel{\Delta_{N}}{\longrightarrow} A_{N} \otimes \mathbb{K}\left[\mathrm{GL}_{N}\right] \stackrel{\text { id } \otimes \mu}{\longrightarrow} A_{N} \otimes \mathbb{K} \simeq A_{N} .
$$

Since $\Delta_{N}$ is an algebra homomorphism and $\mu$ is a derivation, the composite map is a derivation, and we obtain an action of $\operatorname{Lie}\left(\mathrm{GL}_{N}\right)$ on $A_{N}$ by derivations.

The Lie algebra $\mathfrak{g}_{N}=\mathfrak{g l}_{N}(\mathbb{K})$ can be identified with Lie $\left(\mathrm{GL}_{N}\right)$ by sending any $w \in \mathfrak{g}_{N}$ to the linear map $\mu_{w}: \mathbb{K}\left[\mathrm{GL}_{N}\right] \rightarrow \mathbb{K}$ defined by

$$
\mu_{w}(x)=q(x(1+\varepsilon w)) \quad \text { for all } x \in \mathbb{K}\left[\mathrm{GL}_{N}\right]
$$


where $\varepsilon$ is the generator of the algebra of dual numbers $\mathbb{K}[\varepsilon] /\left(\varepsilon^{2}\right), x(1+\varepsilon w)$ is the evaluation of $x$ at $1+\varepsilon w \in \mathrm{GL}_{N}\left(\mathbb{K}[\varepsilon] /\left(\varepsilon^{2}\right)\right)$ and $q: \mathbb{K}[\varepsilon] /\left(\varepsilon^{2}\right) \rightarrow \mathbb{K}$ is defined by $q(k+l \varepsilon)=l$ for all $k, l \in \mathbb{K}$. Through this identification, the action of $\operatorname{Lie}\left(\mathrm{GL}_{N}\right)$ on $A_{N}$ determines an action of $\mathfrak{g}_{N}$ on $A_{N}$. For any $w \in \mathfrak{g}_{N}, a \in A$ and $i, j \in\{1, \ldots, N\}$,

$$
\begin{array}{rll}
w a_{i j}=\left(\mathrm{id} \otimes \mu_{w}\right) \Delta_{N}\left(a_{i j}\right) & \stackrel{\text { A.0.4 }}{=} & \mu_{w}\left(\bar{x}_{i k} x_{l j}\right) a_{k l} \\
& = & q\left(\bar{x}_{i k}(1+\varepsilon w) x_{l j}(1+\varepsilon w)\right) a_{k l} \\
& = & q\left(\left(\delta_{i k}-\varepsilon w_{i, k}\right)\left(\delta_{l j}+\varepsilon w_{l, j}\right)\right) a_{k l} \\
= & \left(-w_{i, k} \delta_{l j}+\delta_{i k} w_{l, j}\right) a_{k l}=-w_{i, k} a_{k j}+a_{i l} w_{l, j} .
\end{array}
$$

Thus, this action of $\mathfrak{g}_{N}$ coincides with the action (3.2.3).

Lemma A.1 has the following useful consequence. If $\mathbb{K}$ is an algebraically closed field, then $A_{N}^{G_{N}}$ coincides with the $\mathrm{GL}_{N}$-invariant part of $A_{N}$ as a representation of the group scheme $\mathrm{GL}_{N}$, see [Ja, $\left.\S I .2 .8\right]$. Therefore $A_{N}^{G_{N}} \subset A_{N}^{\mathfrak{g}_{N}}$.

\section{Appendix B. Comparison of QUASI-Poisson struCtures}

We compare our quasi-Poisson structure on representation manifolds with those defined in AKsM. We assume that $\mathbb{K}=\mathbb{R}$ and use notations of Section 8 . In order to make the computations of AKsM compatible with our conventions, we multiply the quasi-Poisson bivector fields appearing in [AKsM] by 2 .

B.1. Preliminaries. In this subsection, $G$ is a Lie group whose Lie algebra $\mathfrak{g}$ is endowed with a $G$-invariant non-degenerate symmetric bilinear form $\cdot: \mathfrak{g} \times \mathfrak{g} \rightarrow \mathbb{R}$. The construction of AKsM uses three main ingredients: a so-called fusion product of quasi-Poisson manifolds, a canonical quasi-Poisson structure on $G$ and a preferred quasi-Poisson structure on $G \times G$. We briefly recall the relevant definitions.

The fusion product is defined as follows. Let $\mathfrak{d}=\mathfrak{g} \oplus \mathfrak{g}$ be the Lie algebra of the Lie group $D=G \times G$. Pick a basis $\left(e_{i}\right)_{i}$ of $\mathfrak{g}$, let $\left(e_{i}^{\sharp}\right)_{i}$ be the basis of $\mathfrak{g}$ dual to $\left(e_{i}\right)_{i}$ with respect to the form $\cdot$ and set

$$
\psi=\sum_{i}\left(e_{i}^{\sharp}, 0\right) \wedge\left(0, e_{i}\right) \in \Lambda^{2} \mathfrak{d} .
$$

The bivector $\psi$ is independent of the choice of the basis $\left(e_{i}\right)_{i}$ because it corresponds to the skew-symmetric bilinear pairing

$$
\mathfrak{d} \times \mathfrak{d} \longrightarrow \mathbb{R},\left(\left(v_{1}, v_{2}\right),\left(w_{1}, w_{2}\right)\right) \longmapsto v_{1} \cdot w_{2}-v_{2} \cdot w_{1}
$$

through the canonical isomorphism $\Lambda^{2} \mathfrak{d} \simeq \Lambda^{2} \mathfrak{d}^{*}$ induced by the form $\cdot: \mathfrak{g} \times \mathfrak{g} \rightarrow \mathbb{R}$.

Consider $G$-manifolds $M_{1}$ and $M_{2}$ equipped with quasi-Poisson bivector fields $P_{1}$ and $P_{2}$, respectively. Let $\operatorname{pr}_{i}: M_{1} \times M_{2} \rightarrow M_{i}$ be the $i$-th projection for $i=1,2$. We identify $T\left(M_{1} \times M_{2}\right)$ with $\operatorname{pr}_{1}^{*}\left(T\left(M_{1}\right)\right) \oplus \operatorname{pr}_{2}^{*}\left(T\left(M_{2}\right)\right)$ where $T$ stands for the tangent bundle of a manifold. The Lie group $D=G \times G$ acts on $M_{1} \times M_{2}$ and, under this action, the bivector $\psi \in \Lambda^{2} \mathfrak{d}$ generates a bivector field $\psi_{M_{1} \times M_{2}}$ of $M_{1} \times M_{2}$. If we endow $M_{1} \times M_{2}$ with the diagonal $G$-action, then

$$
P_{1} \circledast P_{2}=\operatorname{pr}_{1}^{*}\left(P_{1}\right)+\operatorname{pr}_{2}^{*}\left(P_{2}\right)-\psi_{M_{1} \times M_{2}}
$$

is a quasi-Poisson bivector field on $M_{1} \times M_{2}$, see AKsM, §5].

For any $v \in \mathfrak{g}$, denote by $v_{G}^{L}$ the vector field on $G$ generated by $v$ through the left action of $G$ on itself by $(g, m) \mapsto m g^{-1}$. Similarly, denote by $v_{G}^{R}$ the vector field on $G$ generated by $v$ through the left action of $G$ on itself by $(g, m) \mapsto g m$. Note 
that the vector field $v_{G}^{L}$ is left-invariant while $v_{G}^{R}$ is right-invariant. (Our notation differs from that of [AKsM] where $v_{G}^{L}$ is denoted by $v^{L}$ and $v_{G}^{R}$ is denoted by $-v^{R}$.) Then

$$
P_{G}=\sum_{i}\left(e_{i}\right)_{G}^{L} \wedge\left(e_{i}^{\sharp}\right)_{G}^{R}
$$

is a quasi-Poisson bivector field on the underlying manifold of $G$ endowed with left action of $G$ by conjugations, see $\left[\mathrm{AKsM}\right.$, $\S 3$ ]. Note that $P_{G}$ does not depend on the choice of the basis $\left(e_{i}\right)_{i}$ of $\mathfrak{g}$ since it is the bivector field generated by $-\psi$ when the Lie group $D$ acts on $G$ by $\left(\left(g_{1}, g_{2}\right), g\right) \mapsto g_{1} g g_{2}^{-1}$.

Let $G$ act diagonally on the left of $D=G \times G$ by conjugations. Then

$$
\begin{aligned}
P_{D}= & -\sum_{i} \operatorname{pr}_{1}^{*}\left(e_{i}\right)_{G}^{L} \wedge \operatorname{pr}_{2}^{*}\left(e_{i}^{\sharp}\right)_{G}^{R}-\sum_{i} \operatorname{pr}_{1}^{*}\left(e_{i}\right)_{G}^{R} \wedge \operatorname{pr}_{2}^{*}\left(e_{i}^{\sharp}\right)_{G}^{L} \\
& -\sum_{i}\left(\operatorname{pr}_{1}^{*}\left(e_{i}\right)_{G}^{R}+\operatorname{pr}_{2}^{*}\left(e_{i}\right)_{G}^{L}\right) \wedge\left(\operatorname{pr}_{1}^{*}\left(e_{i}^{\sharp}\right)_{G}^{L}+\operatorname{pr}_{2}^{*}\left(e_{i}^{\sharp}\right)_{G}^{R}\right)
\end{aligned}
$$

is a quasi-Poisson bivector field on $D$, see AKsM Examples $5.3 \&$ \&.4]. The idea behind the definition of $P_{D}$ is as follows. There is a general procedure which transforms a quasi-Poisson $(G \times G)$-manifold into a quasi-Poisson $G$-manifold and which generalizes the fusion product of quasi-Poisson $G$-manifolds, see AKsM, Proposition 5.1]. Applying this procedure three times to the manifold $D=G \times G$ (where $G^{4}$ acts by the left/right multiplication on the two factors), one obtains $P_{D}$.

B.2. Comparison. Assume that $G=\mathrm{GL}_{N}(\mathbb{R})$ with $N \geq 1$. The Lie algebra $\mathfrak{g}=\mathfrak{g l}_{N}(\mathbb{R})$ of $G$ is equipped with the trace form defined by $v \cdot w=\operatorname{tr}(v w)$ for any $v, w \in \mathfrak{g}$. Let $\Sigma$ be a compact connected oriented surface of genus $g \geq 0$ with $m+1 \geq 1$ boundary components. We choose a base point $* \in \partial \Sigma$, and fix a basis $\left(p_{1}, q_{1}, \ldots, p_{g}, q_{g}, z_{1}, \ldots, z_{m}\right)$ of the free group $\pi=\pi_{1}(\Sigma, *)$ as shown on Figure 1 We use this basis to identify $\mathcal{H}=\operatorname{Hom}(\pi, G)$ with $D^{g} \times G^{m}$ where $D=G \times G$. By [AKsM], we have the following quasi-Poisson bivector field on $\mathcal{H}$ :

$$
P^{\prime}=\underbrace{P_{D} \circledast \cdots \circledast P_{D}}_{g \text { times }} \circledast \underbrace{P_{G} \circledast \cdots \circledast P_{G}}_{m \text { times }} .
$$

The following theorem shows that the resulting quasi-Poisson manifold $\left(\mathcal{H}, P^{\prime}\right)$ coincides with the quasi-Poisson manifold $(\mathcal{H}, P)$ produced by Theorem 3.1

Theorem B.1. The bivector field $P^{\prime}$ is equal to the bivector field $P$ of Theorem 8.2.

Proof. We first verify the equality $P^{\prime}=P$ in the case where $g=0$ and $m=1$. Then the group $\pi$ is freely generated by $z=z_{1}$ and $\mathcal{H}=G$. Since the elementary matrices $f_{i j}$ and $f_{j i}$ (with $i, j \in\{1, \ldots, N\}$ ) provide dual bases of $\mathfrak{g}$, (B.1.3) gives

$$
P^{\prime}=P_{G}=\left(f_{r s}\right)_{G}^{L} \wedge\left(f_{s r}\right)_{G}^{R}
$$

so that, for any $i, j, k, l \in\{1, \ldots, N\}$,

$$
\left\{\tilde{z}_{i j}, \tilde{z}_{k l}\right\}_{P^{\prime}}=\left(f_{r s}\right)_{G}^{L}\left(\tilde{z}_{i j}\right)\left(f_{s r}\right)_{G}^{R}\left(\tilde{z}_{k l}\right)-\left(f_{r s}\right)_{G}^{L}\left(\tilde{z}_{k l}\right)\left(f_{s r}\right)_{G}^{R}\left(\tilde{z}_{i j}\right) .
$$

Moreover we have

$$
\left(f_{s r}\right)_{G}^{R}\left(\tilde{z}_{i j}\right)=-\delta_{i s} \tilde{z}_{r j} \quad \text { and } \quad\left(f_{r s}\right)_{G}^{L}\left(\tilde{z}_{i j}\right)=\delta_{j s} \tilde{z}_{i r}
$$

since, at any point $m \in G$,

$$
\left(f_{s r}\right)_{G}^{R}\left(\tilde{z}_{i j}\right)(m)=\left.\frac{d}{d t}\right|_{t=0} \tilde{z}_{i j}\left(e^{-t f_{s r}} m\right)=-\tilde{z}_{i j}\left(f_{s r} m\right)=-\delta_{i s} m_{r j}
$$




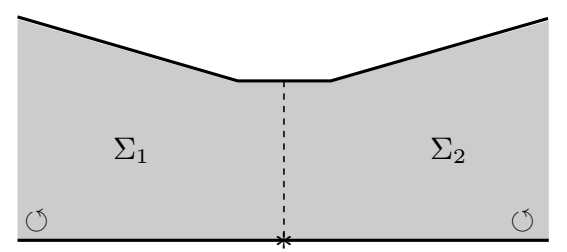

Figure 2. The boundary connected sum $\Sigma_{0}$ of $\Sigma_{1}$ and $\Sigma_{2}$

$$
\left(f_{r s}\right)_{G}^{L}\left(\tilde{z}_{i j}\right)(m)=\left.\frac{d}{d t}\right|_{t=0} \tilde{z}_{i j}\left(m e^{t f_{r s}}\right)=\tilde{z}_{i j}\left(m f_{r s}\right)=\delta_{j s} m_{i r} .
$$

We deduce that

$$
\begin{aligned}
\left\{\tilde{z}_{i j}, \tilde{z}_{k l}\right\}_{P^{\prime}} & =\left(\delta_{j s} \tilde{z}_{i r}\right)\left(-\delta_{k s} \tilde{z}_{r l}\right)-\left(\delta_{l s} \tilde{z}_{k r}\right)\left(-\delta_{i s} \tilde{z}_{r j}\right) \\
& =-\delta_{j k} \tilde{z}_{i r} \tilde{z}_{r l}+\delta_{i l} \tilde{z}_{k r} \tilde{z}_{r j} \stackrel{8.3 .2}{=}\left\{\tilde{z}_{i j}, \tilde{z}_{k l}\right\}_{P} .
\end{aligned}
$$

Next, we verify the equality $P^{\prime}=P$ in the case where $g=1$ and $m=0$. Then the group $\pi$ is freely generated by $(p, q)=\left(p_{1}, q_{1}\right)$ and $\mathcal{H}=D$. We have

$$
\begin{aligned}
P^{\prime}=P_{D}= & -\operatorname{pr}_{1}^{*}\left(f_{r s}\right)_{G}^{L} \wedge \operatorname{pr}_{2}^{*}\left(f_{s r}\right)_{G}^{R}-\operatorname{pr}_{1}^{*}\left(f_{r s}\right)_{G}^{R} \wedge \operatorname{pr}_{2}^{*}\left(f_{s r}\right)_{G}^{L} \\
& -\operatorname{pr}_{1}^{*}\left(f_{r s}\right)_{G}^{R} \wedge \operatorname{pr}_{2}^{*}\left(f_{s r}\right)_{G}^{R}+\operatorname{pr}_{1}^{*}\left(f_{r s}\right)_{G}^{L} \wedge \operatorname{pr}_{2}^{*}\left(f_{s r}\right)_{G}^{L} \\
& +\operatorname{pr}_{1}^{*}\left(f_{r s}\right)_{G}^{L} \wedge \operatorname{pr}_{1}^{*}\left(f_{s r}\right)_{G}^{R}-\operatorname{pr}_{2}^{*}\left(f_{r s}\right)_{G}^{L} \wedge \operatorname{pr}_{2}^{*}\left(f_{s r}\right)_{G}^{R} .
\end{aligned}
$$

By computations similar to the previous case, we have for any $i, j, k, l \in\{1, \ldots, N\}$

$$
\begin{aligned}
& \left\{\tilde{p}_{i j}, \tilde{p}_{k l}\right\}_{P^{\prime}}=-\delta_{j k} \tilde{p}_{i r} \tilde{p}_{r l}+\delta_{i l} \tilde{p}_{k r} \tilde{p}_{r j} \stackrel{8.3 .40}{=}\left\{\tilde{p}_{i j}, \tilde{p}_{k l}\right\}_{P} \\
& \left\{\tilde{q}_{i j}, \tilde{q}_{k l}\right\}_{P^{\prime}}=-\left(-\delta_{j k} \tilde{q}_{i r} \tilde{q}_{r l}+\delta_{i l} \tilde{q}_{k r} \tilde{q}_{r j}\right) \stackrel{8.3 .5}{=}\left\{\tilde{q}_{i j}, \tilde{q}_{k l}\right\}_{P} .
\end{aligned}
$$

Moreover,

$$
\begin{aligned}
\left\{\tilde{p}_{i j}, \tilde{q}_{k l}\right\}_{P^{\prime}}= & -\left(f_{r s}\right)_{G}^{L}\left(\tilde{p}_{i j}\right)\left(f_{s r}\right)_{G}^{R}\left(\tilde{q}_{k l}\right)-\left(f_{r s}\right)_{G}^{R}\left(\tilde{p}_{i j}\right)\left(f_{s r}\right)_{G}^{L}\left(\tilde{q}_{k l}\right) \\
& -\left(f_{r s}\right)_{G}^{R}\left(\tilde{p}_{i j}\right)\left(f_{s r}\right)_{G}^{R}\left(\tilde{q}_{k l}\right)+\left(f_{r s}\right)_{G}^{L}\left(\tilde{p}_{i j}\right)\left(f_{s r}\right)_{G}^{L}\left(\tilde{q}_{k l}\right) \\
\stackrel{\text { B.2.1. }}{=} & -\left(\delta_{j s} \tilde{p}_{i r}\right)\left(-\delta_{k s} \tilde{q}_{r l}\right)-\left(-\delta_{i r} \tilde{p}_{s j}\right)\left(\delta_{l r} \tilde{q}_{k s}\right) \\
& -\left(-\delta_{i r} \tilde{p}_{s j}\right)\left(-\delta_{k s} \tilde{q}_{r l}\right)+\left(\delta_{j s} \tilde{p}_{i r}\right)\left(\delta_{l r} \tilde{q}_{k s}\right) \\
= & \delta_{j k} \tilde{p}_{i r} \tilde{q}_{r l}+\delta_{i l} \tilde{p}_{s j} \tilde{q}_{k s}-\tilde{p}_{k j} \tilde{q}_{i l}+\tilde{p}_{i l} \tilde{q}_{k j} \stackrel{\text { 8.3.6l) }}{=}\left\{\tilde{p}_{i j}, \tilde{q}_{k l}\right\}_{P} .
\end{aligned}
$$

Theorem B.1 follows now from the two cases above and the following claim.

Claim. Let $\Sigma_{0}$ be a compact connected oriented surface with base point $* \in \partial \Sigma_{0}$ which is the "boundary connected sum" of two compact connected oriented surfaces $\Sigma_{1}$ and $\Sigma_{2}$, see Figure 2. For each $i \in\{0,1,2\}$, let $P_{i}$ be the quasi-Poisson bivector field on $\mathcal{H}_{i}=\operatorname{Hom}\left(\pi_{1}\left(\Sigma_{i}, *\right), G\right)$ produced by Theorem [8.2. Then $\mathcal{H}_{0}=\mathcal{H}_{1} \times \mathcal{H}_{2}$ and $P_{0}=P_{1} \circledast P_{2}$.

For $x \in \pi_{1}\left(\Sigma_{0}, *\right)$ and $i, j \in\{1, \ldots, N\}$, let $\tilde{x}_{i j} \in C^{\infty}\left(\mathcal{H}_{0}\right)$ be the function carrying any $h \in \mathcal{H}_{0}$ to the $(i, j)$-th coefficient of the matrix $h(x)$. In order to verify the claim, it is enough to check that

$$
\left\{\tilde{x}_{i j}, \tilde{y}_{k l}\right\}_{P_{0}}=\left\{\tilde{x}_{i j}, \tilde{y}_{k l}\right\}_{P_{1} \circledast P_{2}}
$$


GWÉNAËL MASSUYEAU AND VLADIMIR TURAEV

for any $x, y \in \pi_{1}\left(\Sigma_{0}, *\right)$ and $i, j, k, l \in\{1, \ldots, N\}$. If both $x, y$ belong to $\pi_{1}\left(\Sigma_{j}, *\right)$ for some $j \in\{1,2\}$, then (B.2.2) follows from the equality $\left\{\tilde{x}_{i j}, \tilde{y}_{k l}\right\}_{P_{0}}=\left\{\tilde{x}_{i j}, \tilde{y}_{k l}\right\}_{P_{j}}$ (since $\{\{x, y\}\}^{s}$ can be fully computed in $\Sigma_{j}$ ) and the fact that $\left\{\tilde{x}_{i j}, \tilde{y}_{k l}\right\}_{P_{1} \circledast P_{2}}=$ $\left\{\tilde{x}_{i j}, \tilde{y}_{k l}\right\}_{P_{j}}$ (since the projection $\mathcal{H}_{1} \times \mathcal{H}_{2} \rightarrow \mathcal{H}_{j}$ carries $P_{1} \circledast P_{2}$ to $P_{j}$ ). It remains to consider the case where $x \in \pi_{1}\left(\Sigma_{1}, *\right)$ and $y \in \pi_{1}\left(\Sigma_{2}, *\right)$.

Since $\{\{x, y\}\}^{\eta}=0$, we have $\{\{x, y\}\}^{s}=1 \otimes x y+y x \otimes 1-x \otimes y-y \otimes x$ so that

$$
\left\{\tilde{x}_{i j}, \tilde{y}_{k l}\right\}_{P_{0}}=\delta_{k j} \tilde{x}_{i r} \tilde{y}_{r l}+\tilde{y}_{k s} \tilde{x}_{s j} \delta_{i l}-\tilde{x}_{k j} \tilde{y}_{i l}-\tilde{y}_{k j} \tilde{x}_{i l} .
$$

Besides we have

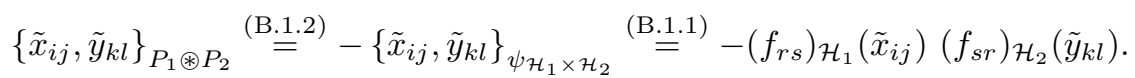

At any point $m \in \mathcal{H}_{1}$,

$$
\begin{aligned}
\left(f_{r s}\right)_{\mathcal{H}_{1}}\left(\tilde{x}_{i j}\right)(m) & =\left.\frac{d}{d t}\right|_{t=0} \tilde{x}_{i j}\left(e^{-t f_{r s}} m e^{t f_{r s}}\right) \\
& =-\tilde{x}_{i j}\left(f_{r s} m\right)+\tilde{x}_{i j}\left(m f_{r s}\right)=-\delta_{i r} \tilde{x}_{s j}(m)+\delta_{j s} \tilde{x}_{i r}(m)
\end{aligned}
$$

and, by a similar computation, at any point $n \in \mathcal{H}_{2}$,

$$
\left(f_{s r}\right)_{\mathcal{H}_{2}}\left(\tilde{y}_{k l}\right)(n)=-\delta_{k s} \tilde{y}_{r l}(n)+\delta_{l r} \tilde{y}_{k s}(n) .
$$

Therefore

$$
\begin{aligned}
\left\{\tilde{x}_{i j}, \tilde{y}_{k l}\right\}_{P_{1} \circledast P_{2}} & =-\left(-\delta_{i r} \tilde{x}_{s j}+\delta_{j s} \tilde{x}_{i r}\right)\left(-\delta_{k s} \tilde{y}_{r l}+\delta_{l r} \tilde{y}_{k s}\right) \\
& =-\tilde{x}_{k j} \tilde{y}_{i l}+\delta_{j k} \tilde{x}_{i r} \tilde{y}_{r l}+\delta_{i l} \tilde{x}_{s j} \tilde{y}_{k s}-\tilde{x}_{i l} \tilde{y}_{k j} \stackrel{\text { B.2.3) }}{=}\left\{\tilde{x}_{i j}, \tilde{y}_{k l}\right\}_{P_{0}} \cdot
\end{aligned}
$$

\section{REFERENCES}

[AKsM] A. Alekseev, Y. Kosmann-Schwarzbach, E. Meinrenken, Quasi-Poisson manifolds. Canad. J. Math. 54 (2002), no. 1, 3-29.

[AMM] A. Alekseev, A. Malkin, E. Meinrenken, Lie group valued moment maps. J. Differential Geom. 48 (1998), no. 3, 445-495.

[Au] M. Audin, Lectures on gauge theory and integrable systems. Gauge theory and symplectic geometry (Montreal, PQ, 1995), 1-48, NATO Adv. Sci. Inst. Ser. C Math. Phys. Sci., 488, Kluwer Acad. Publ., Dordrecht, 1997.

[CS] M. Chas, D. Sullivan, String Topology. Preprint (1999) arXiv:math/9911159.

[Cb] W. Crawley-Boevey, Poisson structures on moduli spaces of representations. J. Algebra 325 (2011), 205-215.

[Do] I. V. Dolgachev, Introduction to algebraic geometry. Lecture notes available at http://www.math.lsa.umich.edu/ idolga/lecturenotes.html

[FR] V. V. Fock, A. A. Rosly, Poisson structure on moduli of flat connections on Riemann surfaces and the r-matrix. (Russian) Moscow Seminar in Math. Physics. English translation: Amer. Math. Soc. Transl. Ser. 2, 191, 67-86 (1999).

[Go1] W. M. Goldman, The symplectic nature of fundamental groups of surfaces. Adv. in Math. 54 (1984), no. 2, 200-225.

[Go2] W. M. Goldman, Invariant functions on Lie groups and Hamiltonian flows of surface group representations. Invent. Math. 85 (1986), no. 2, 263-302.

[Go3] W. M. Goldman, Mapping class group dynamics on surface group representations. Problems on mapping class groups and related topics, 189-214, Proc. Sympos. Pure Math., 74, Amer. Math. Soc., Providence, RI, 2006.

[GHJW] K. Guruprasad, J. Huebschmann, L. Jeffrey, A. Weinstein, Group systems, groupoids, and moduli spaces of parabolic bundles. Duke Math. J. 89 (1997), no. 2, 377-412.

$[\mathrm{Hu}] \mathrm{J}$. Huebschmann, Poisson geometry of certain moduli spaces. Rend. Circ. Mat. Palermo (2) Suppl. No. 39 (1996), 15-35.

[Ja] J. C. Jantzen, Representations of algebraic groups. Second edition. Mathematical Surveys and Monographs, 107. American Mathematical Society, Providence, RI, 2003. 
[KK1] N. Kawazumi, Y. Kuno, The logarithms of Dehn twists. Preprint (2010) arXiv: 1008.5017.

[KK2] N. Kawazumi, Y. Kuno, Intersections of curves on surfaces and their applications to mapping class groups. Preprint (2011) arXiv:1112.3841.

[La] S. Lawton, Poisson geometry of $\mathrm{SL}(3, \mathbb{C})$-character varieties relative to a surface with boundary. Trans. Amer. Math. Soc. 361 (2009), no. 5, 2397-2429.

[LbP] L. Le Bruyn, C. Procesi, Semisimple representations of quivers. Trans. Amer. Math. Soc. 317 (1990), no. 2, 585-598.

[Mar] C.-M. Marle, The Schouten-Nijenhuis bracket and interior products. J. Geom. Phys. 23 (1997), no. 3-4, 350-359.

[Mas] G. Massuyeau, Infinitesimal Morita homomorphisms and the tree-level of the LMO invariant. Bull. Soc. Math. France 140:1 (2012), 101-161.

[MT] G. Massuyeau, V. Turaev, Fox pairings and generalized Dehn twists. Preprint (2011) arXiv: 1109.5248.

[Pa] C. D. Papakyriakopoulos, Planar regular coverings of orientable closed surfaces. Knots, groups, and 3-manifolds (Papers dedicated to the memory of R. H. Fox), 261-292. Ann. of Math. Studies, No. 84, Princeton Univ. Press, Princeton, N.J., 1975.

[Pe $] \quad$ B. Perron, A homotopic intersection theory on surfaces: applications to mapping class group and braids. Enseign. Math. (2) 52 (2006), no. 1-2, 159-186.

[Tu] V. G. Turaev, Intersections of loops in two-dimensional manifolds. (Russian) Mat. Sb. 106(148) (1978), 566-588. English translation: Math. USSR, Sb. 35 (1979), 229-250.

[VdB] M. Van den Bergh, Double Poisson algebras. Trans. Amer. Math. Soc. 360 (2008), no. $11,5711-5769$.

[Wo] S. Wolpert, On the symplectic geometry of deformations of a hyperbolic surface. Ann. of Math. (2) 117 (1983), 207-234.

GwÉnaËL Massuyeau

IRMA, Université de Strasbourg \& CNRS

7 Rue René Descartes

67084 Strasbourg, France

massuyeau@math.unistra.fr

Vladimir Turaev

Department of Mathematics

INDIANA UNIVERSITY

BLOOMINGTON IN47405, USA

vturaev@yahoo.com 